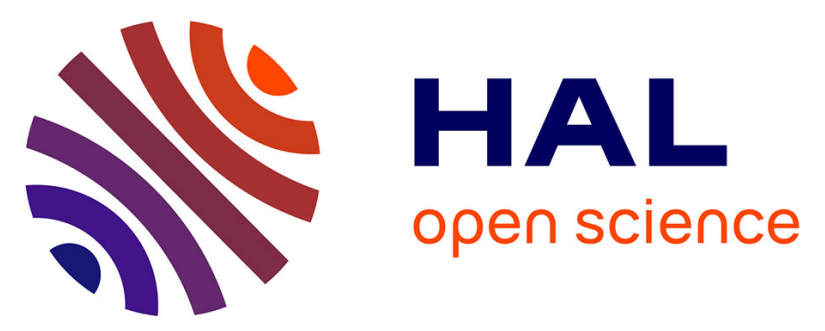

\title{
Tubulin glycylation controls axonemal dynein activity, flagellar beat, and male fertility
}

Sudarshan Gadadhar, Gonzalo Alvarez Viar, Jan Niklas Hansen, An Gong, Aleksandr Kostarev, Côme Ialy-Radio, Sophie Leboucher, Marjorie Whitfield, Ahmed Ziyyat, Aminata Touré, et al.

\section{To cite this version:}

Sudarshan Gadadhar, Gonzalo Alvarez Viar, Jan Niklas Hansen, An Gong, Aleksandr Kostarev, et al.. Tubulin glycylation controls axonemal dynein activity, flagellar beat, and male fertility. Science, 2021, 371 (6525), pp.eabd4914. 10.1126/science.abd4914 . hal-03323742

\section{HAL Id: hal-03323742 \\ https://hal.science/hal-03323742}

Submitted on 22 Aug 2021

HAL is a multi-disciplinary open access archive for the deposit and dissemination of scientific research documents, whether they are published or not. The documents may come from teaching and research institutions in France or abroad, or from public or private research centers.
L'archive ouverte pluridisciplinaire HAL, est destinée au dépôt et à la diffusion de documents scientifiques de niveau recherche, publiés ou non, émanant des établissements d'enseignement et de recherche français ou étrangers, des laboratoires publics ou privés. 


\section{Tubulin glycylation controls axonemal dynein activity, flagellar beat and}

\section{male fertility}

Sudarshan Gadadhar", Gonzalo Alvarez Viar*, Jan Niklas Hansen*, An Gong*, Aleksandr Kostarev, Côme Ialy-Radio, Sophie Leboucher, Marjorie Whitfield, Ahmed Ziyyat, Aminata

Touré, Luis Alvarez*\#, Gaia Pigino*\#, Carsten Janke"

\section{Introduction}

Microtubules are key components of the eukaryotic cytoskeleton. While being involved in a wide variety of functions, microtubules are structurally highly similar across most cell types and organisms. It was suggested that a "tubulin code", formed by combinations of tubulin posttranslational modifications adapts individual microtubules to specific functions within living cells. However, clear-cut functional and mechanistic data verifying this concept are still scarce. Glycylation is among the least explored posttranslational modifications of tubulin and has, so far, exclusively been found on microtubules of cilia and flagella from a variety of species. Previous work suggested that glycylation might be essential for cilia and flagella, but mechanistic insight remains lacking.

\section{Rationale}

Two enzymes from the tubulin-tyrosine ligase-like (TTLL) family, TTLL3 and TTLL8, are essential to initiate glycylation of tubulin in mammals. To entirely abolish glycylation at the organism level, and to determine its physiological function, we generated a double-knockout mouse lacking both glycylating enzymes $\left(\right.$ Ttll3 $^{--}$Ttll8 $\left.8^{--}\right)$. Inactivation of these two enzymes indeed led to a lack of glycylation in all analysed cilia and flagella. This allowed us to investigate the role of glycylation in the function of these organelles.

\section{Results}

Despite the absence of glycylation in $T t l l 3^{-/} \mathrm{Ttll}^{-/-}$mice, no gross defects were observed at the organism and tissue levels. Motile ependymal cilia in brain ventricles, as well as motile cilia in the respiratory tract were present and appeared normal. Sperm flagella were also assembled normally, and sperm were able to swim. However, in-vitro fertility assays showed that male $T t l l 3^{-/}$Ttll $^{-/}$mice were subfertile. Computer-assisted sperm analyses revealed motility defects of $T t l l 3^{-/} \mathrm{Ttll}^{-/}$sperm. Further analyses showed that lack of glycylation leads 
to perturbed flagellar beat patterns, which caused $T t l l 3^{-/} T_{t l l 8^{-/}}$sperm to swim predominantly along circular paths. This is highly unusual for mammalian sperm and interferes with their ability to reach the oocyte for fertilisation. To determine the molecular mechanisms underlying this aberrant flagellar beat, we used cryo-electron tomography. The 3D structure

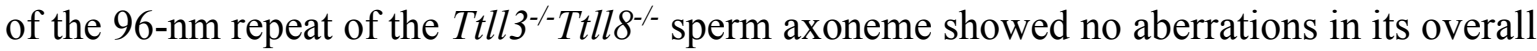
assembly. By contrast, the structure of both outer and inner dynein arms (ODAs, IDAs) was perturbed in $\mathrm{Ttll}^{-/-} \mathrm{Ttll}^{-/-}$flagella. Classification analysis showed that the incidence and distribution of pre-powerstroke and post-powerstroke conformations of ODAs and IDAs were altered in $\mathrm{Ttll}^{-/-} \mathrm{Ttll}^{-/-}$sperm. This indicates that glycylation is required to efficiently control the dynein powerstroke cycle, which is essential for the generation of a physiological flagellar beat.

\section{Conclusion}

Our work indicates that tubulin glycylation regulates the beat of mammalian flagella by modulating axonemal dynein motor activity. Lack of glycylation led to perturbed sperm motility and male subfertility in mice. Considering that compared to mice, human sperm are more susceptible to deficiencies in sperm motility, our findings imply that a perturbation of tubulin glycylation could underlie some forms of male infertility in humans.

\section{Figure caption:}

\section{Tubulin glycylation controls sperm motility}

(A) Microtubules in sperm flagella are rich in tubulin posttranslational modifications. Mice deficient for the glycylating enzymes TTLL3 and TTLL8 lack glycylation.

(B) Mammalian sperm swim in linear paths. In the absence of glycylation, abnormal, mostly circular swimming patterns are observed, which impede progressive swimming.

(C) Absence of glycylation leads to perturbed distribution of axonemal dynein conformations in Ttll $^{-/-}$Ttll $^{-/-}$flagella, which impedes normal flagellar beating. 
A wildtype

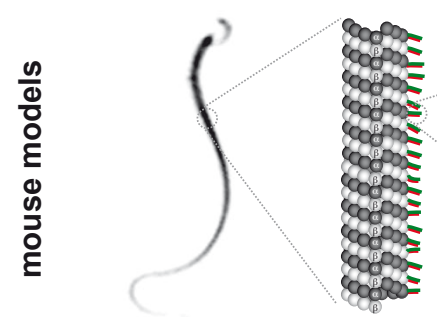

sperm flagellum microtubules are glutamylated \& glycylated

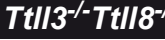
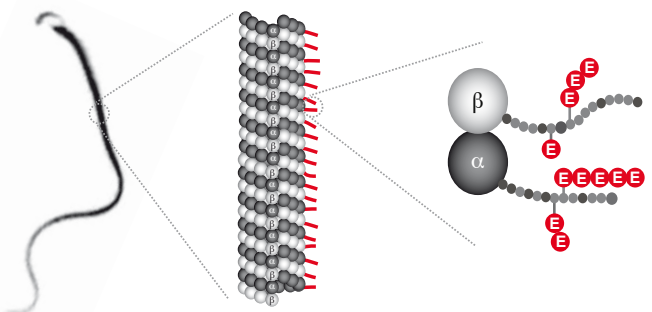

sperm flagellum microtubules are only glutamylated
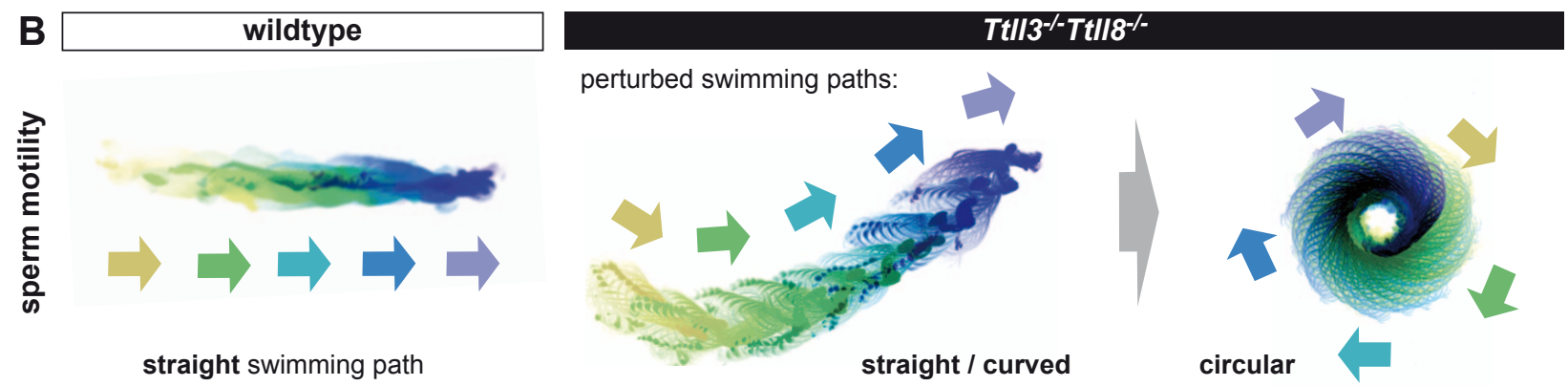

straight swimming path

$$
\text { straight / curved }
$$

circular
C

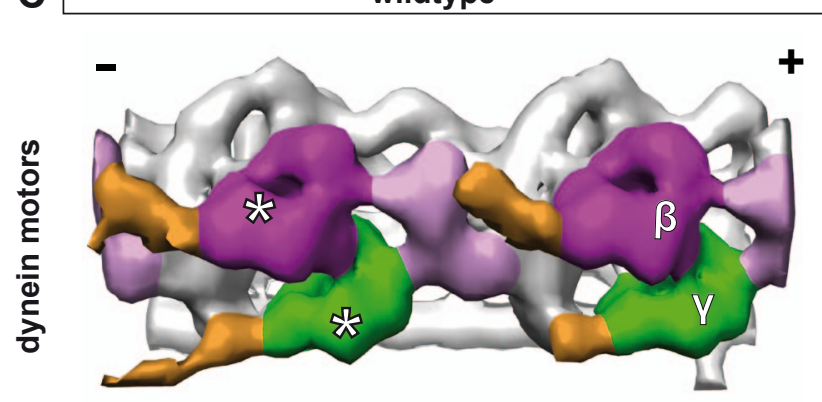

$\beta$-dynein head $\gamma$-dynein head dynein tail

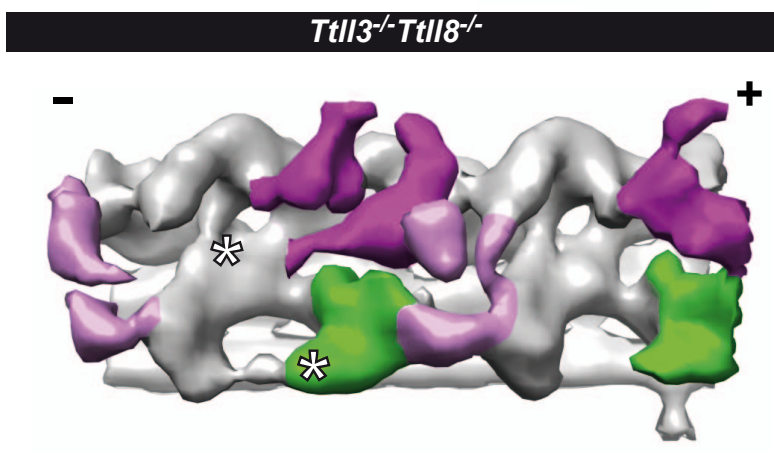

dynein motors are in perturbed conformations 


\section{Tubulin glycylation controls axonemal dynein activity, flagellar beat and}

\section{male fertility}

Sudarshan Gadadhar ${ }^{1,2 \#}$, Gonzalo Alvarez Viar ${ }^{3 *}$, Jan Niklas Hansen ${ }^{4 *}$, An Gong ${ }^{5 *}$, Aleksandr Kostarev ${ }^{3}$, Côme Ialy-Radio ${ }^{6}$, Sophie Leboucher ${ }^{1,2}$, Marjorie Whitfield ${ }^{6}$, Ahmed

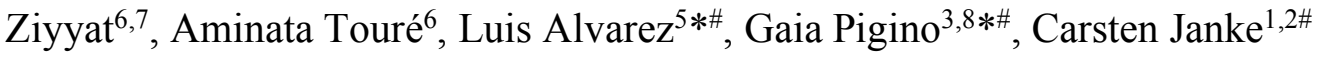

${ }^{1}$ Institut Curie, PSL Research University, CNRS UMR3348, F-91405 Orsay, France

${ }^{2}$ Université Paris Sud, Université Paris-Saclay, CNRS UMR3348, F-91405 Orsay, France

${ }^{3}$ Max Planck Institute of Molecular Cell Biology and Genetics, D-01307 Dresden, Germany

${ }^{4}$ Institute of Innate Immunity, Medical Faculty, University of Bonn, D-53127 Bonn, Germany

${ }^{5}$ Center of Advanced European Studies and Research, D-53175 Bonn, Germany

${ }^{6}$ Université de Paris, Institut Cochin, INSERM, CNRS, F-75014 Paris, France

${ }^{7}$ Service d'histologie, d'embryologie, Biologie de la Reproduction, AP-HP, Hôpital Cochin, F-75014 Paris, France

${ }^{8}$ Human Technopole, I-20157 Milan, Italy

*equal contributions

\#corresponding authors: Sudarshan Gadadhar, Carsten Janke, Institut Curie, PSL Research University, CNRS UMR3348, Centre Universitaire, Bâtiment 110, F-91405 Orsay, France, Gaia Pigino, Max Planck Institute of Molecular Cell Biology and Genetics, Pfotenhauerstraße 108, D-01307 Dresden, Germany, Luis Alvarez, Center of Advanced European Studies and Research, D-53175 Bonn, Germany

Telephone: +331 69863127; Fax: +33 169863017; +49 351 2102450; +492289656354 
Email: Carsten.Janke@curie.fr, Sudarshan.Gadadhar@curie.fr, pigino@mpi-cbg.de, luis.alvarez@caesar.de 


\begin{abstract}
Posttranslational modifications of the microtubule cytoskeleton have emerged as key regulators of cellular functions, and their perturbations have been linked to a growing number of human pathologies. Tubulin glycylation modifies microtubules specifically in cilia and flagella, but its functional and mechanistic roles remain unclear. Here we generated a mouse model entirely lacking tubulin glycylation. Male mice were subfertile owing to aberrant beat patterns of their sperm flagella, which impeded the straight swimming of sperm cells. Using cryo-electron tomography, we showed that lack of glycylation caused abnormal conformations of the dynein arms within sperm axonemes, providing the structural basis for the observed dysfunction. Our findings reveal the importance of microtubule glycylation for controlled flagellar beating, directional sperm swimming, and male fertility.
\end{abstract}

Short title: Glycylation regulates axonemal dyneins

One-sentence summary: Loss of tubulin glycylation affects male fertility owing to sperm motility defects and perturbs axonemal dynein conformations. 
Microtubules (MTs) are ubiquitous cytoskeletal components that are structurally highly similar across most cell types and organisms. Their rich variety of posttranslational modifications (PTMs) has for long been suggested to form a "tubulin code" that allows MTs to adapt to specific functions (1). However, clear-cut functional and mechanistic data verifying this concept are still scarce. Glycylation is among the least explored tubulin PTMs and has, so far, exclusively been found on axonemal MTs of a variety of species (2), but rarely on cytoplasmic MTs. Tubulin glycylation in mammals is catalysed in two steps: the tubulin-tyrosine ligase like (TTLL) glycylating enzymes TTLL3 and TTLL8 add the first glycine residues to unmodified tubulin, while TTLL10 uniquely elongates nascent glycine chains. Absence of elongated glycine chains owing to a constitutively inactive TTLL10 in humans is physiologically tolerated (3), whereas a complete loss of glycylation in mice has damaging effects: Knockout of Ttll3 in combination with shRNA-mediated knockdown of Ttll8 in mouse brain ventricles results in the loss of motile cilia from the affected ependymal cells (4). In retina and colon, two organs that do not express Ttll8, the knockout of Ttll3 alone leads to a progressive shortening of the photoreceptors' connecting cilia followed by retina degeneration (5), and to the partial loss of primary cilia in the colon accompanied by accelerated development of colorectal tumours ( 6$)$. Finally, knocking down TTLL3 in cultured MDCK cells leads to a significant shortening of primary cilia (7). It thus appears that glycylation plays a crucial role in ciliary integrity and function, however, its function in the most specialised mammalian cilium, the sperm flagellum, has remained elusive. Sperm flagella, similar to motile cilia, are extremely susceptible to alterations in the tubulin PTM polyglutamylation (8-11), but glycylation has only been shown to be essential for the integrity of the sperm flagellum in Drosophila melanogaster (3). The unique assembly, structure, and size of Drosophila sperm flagella (12) do not allow direct conclusions on the situation in mammals. 


\section{Loss of tubulin glycylation does not affect ciliogenesis}

To understand the role of glycylation from the physiological to the ultrastructural, mechanistic level, we generated a double-knockout mouse for the two initiating glycylases Ttll3 and Ttll8

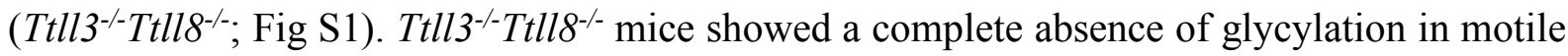
ependymal cilia from brain ventricle walls (Fig S2A,D), motile cilia in the trachea (Fig S2B,D) and sperm flagella (Fig 1A,B), as well as the primary cilia from the kidney collecting tubules (Fig S2C). Cilia and flagella were still present in all tissues analysed (Fig 1A, S2A-C) and moreover, no gross defects were observed at the organism and tissue levels. For instance, the brain and kidneys appeared normal, and there were no signs of hydrocephaly (Fig S3A), or polycystic kidneys (Fig S3B), which are typical signs of ciliary dysfunctions (13). Trachea too showed no aberrations within the multiciliated epithelium (Fig S3C). The absence of ciliary defects in $T t l l 3^{-/-} T_{t l l 8^{-/}}$mice was surprising. In particular, the loss-of-cilia phenotype in ependymal cells, which was induced by shRNA-mediated depletion of TTLL8 in a Ttll3 $3^{--}$ background (4), was not reproduced in $T t l l 3^{--} T_{t l l 8^{-/}}$mice, probably because of compensatory mechanisms during mouse development $(14,15)$.

\section{Absence of glycylation affects sperm motility and male fertility}

Though the overall tissue architecture of testes was not perturbed (Fig S3D), the decreased average litter size of $T t l l 3^{-/}$Ttll $^{-/-}$mice $(6.5 \pm 2.4$ vs. $8.4 \pm 3.9$ for heterozygote controls; Fig $2 \mathrm{~A}$, $\mathrm{S} 3 \mathrm{E}$ ) hinted at the possibility that mice were subfertile. To test specifically for male subfertility, we used in vitro fertilisation assays, which provide a more sensitive readout as compared to in vivo fertilisation (16). Indeed, the fertilisation index of $T t l l 3^{--}$Ttll8 $8^{--}$sperm in vitro was about four times lower as compared to wildtype (Fig 2B, S4B), which establishes the subfertility of sperm lacking tubulin glycylation. 
To determine whether lack of glycylation affects the fertilisation potential of Ttll $^{--}$Ttll $^{-/-}$sperm we investigated three major parameters that govern the quality of sperm isolated from the cauda epididymides: sperm cell count, morphology, and motility (17). Counts and viability of sperm from Ttll3 $^{-/}$Ttll8 $8^{--}$mice were nearly identical to controls (Fig S3F,G Table S1), and only some sperm cells showed morphological defects (Fig S3H). By contrast, most of the motility parameters assessed with computer-assisted sperm analyses (CASA; Fig S4A) (18) were changed in $T t l l 3^{-/} T t l l 8^{-/}$sperm as compared to wildtype (Fig 2C, S4C; Table S1). We found a clear decrease in the proportion of progressive sperm, but also in curvilinear velocity (VCL), straight-line velocity (VSL) and average-path velocity (VAP), three kinematic parameters assessing sperm velocity (Fig 2C, S4A). This strongly suggested a reduced fertilisation potential of $T t l l 3^{-/} T_{t l l 8^{-/}}$sperm owing to reduced sperm motility (asthenozoospermia), a common cause of male infertility (19).

\section{Defects in sperm motility are due to altered flagellar beat patterns}

Following the observation of reduced motility of $T t l l 3^{-/}$Ttll $^{-/-}$sperm, we further characterised the flagellar beat of those sperm. We first performed two-dimensional (2D) analyses of single sperm cells tethered with their heads at the glass surface. Wildtype sperm showed the characteristic symmetric flagellar beat, whereas flagella of sperm from $\mathrm{Ttll3}^{-/} \mathrm{Ttll}^{-/-}$mice were beating asymmetrically, thus inducing a rotatory swimming trajectory in one preferential direction (Fig 3A, Movie S1). Analyses of flagellar beat parameters (20) revealed that the mean flagellar curvature for $\mathrm{Ttll3}^{-/-} \mathrm{Ttll}^{-/}$sperm was $\sim 3.3$ times higher than wildtype (Fig 3B, S5AC). Moreover, $T t l l 3^{-/} T t l l 8^{-/}$sperm flagella beat with an $\sim 2.4$ times lower amplitude than wildtype, and their beat is biased towards the open-hook side of the sperm head, which could explain the asymmetric beat (Fig 3C; S5C,D). Another major defect was found for the peak frequencies of the flagellar beat. While in wildtype a homogenous peak frequency was detected

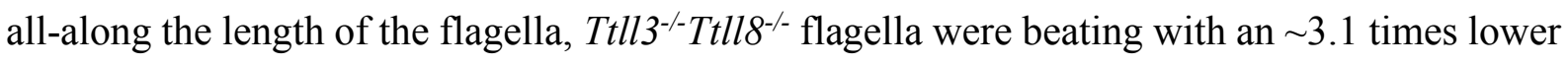


frequency close to the sperm head, but in turn with an $\sim 2.7$ times higher frequency in the rest of the flagellum compared to wildtype sperm (Fig 3D; S5E). Such modifications of beat patterns cannot be explained by premature hyperactivation, a sperm behaviour that normally occurs during sperm transit in the female genital tract, and ensures proper sperm progression towards the oocyte. Hyperactivation would result in a higher beat amplitude and lower beat frequencies, and accordingly, in a higher lateral head displacement (ALH) and a lower beatcross frequency (BCF) in CASA analyses (21-23). Given that ALH was persistently lower and BCF higher for Ttll3 $^{-/}$Ttlls $^{-/}$sperm (Fig 2C), we excluded the presence of premature hyperactivation.

\section{Altered flagellar beat patterns result in anomalous sperm swim paths}

The altered beat patterns we observe for $T t l l 3^{-/} T_{t l l 8^{-/}}$sperm are likely to translate into altered swim paths. We thus simulated the swim paths of sperm in three dimensions (3D) based on the flagellar beat patterns we had determined from tethered sperm (Fig 3A-D), and assuming a flagellar beat that is not perfectly planar (Fig 4A). Our simulations showed that wildtype sperm should swim in a twisted ribbon trajectory (24), whereas $T t l l 3^{--}$Ttll $^{-/-}$sperm would adopt a helical swim path (Fig 4B). To test these predictions, we directly determined the 3D trajectories of free-swimming sperm by in-line holographic microscopy (25). As predicted, wildtype sperm cells swam in a twisted ribbon trajectory, whereas $T t l l 3^{-/} T_{t l l 8^{-/}}$sperm swam predominantly in helical paths (Fig 4C, S6). Both swimming patterns allow sperm to advance, however, when Ttll3 $^{-/-}$Ttll $^{-/-}$sperm reached the wall of the observation chamber, their swimming paths spontaneously switched from helical to circular swimming (Fig 4D), which impedes progressive swimming. Circular swim patterns have been reported for sperm of marine invertebrates (26), but are atypical for mammalian sperm (27). To test the propensity of $\mathrm{Ttll}^{-/-}$ Ttll8 $^{--}$sperm to switch to circular swimming, we recorded the swim paths of large numbers of free-swimming sperm cells near the glass surface of the recording chamber. Under these 
conditions, wildtype sperm swam almost exclusively along straight or curved paths, whereas most $(\sim 86 \%)$ of the Ttll3 $^{-/-}$Ttll $^{-/-}$sperm swam in circular paths (Fig 4E,F; Movie S2). Only a small fraction $(\sim 14 \%)$ of $T t l l 3^{-/} T_{t l l 8^{-/}}$sperm showed incidental progressive movement, but even those switched intermittently to circular movements (Movie S3). The predominantly circular swimming of $T t l l 3^{-/} \mathrm{Ttll}^{-/}$spermatozoa near the glass surface can be explained by the increased average curvature of their flagella (Fig 3B, 4A): mesoscale hydrodynamics simulations predict that a high mean curvature of the flagellar beat contributes to the accumulation of sperm at the surface, prevents sperm rolling, and result in swimming along tight circles (28).

The main defect of Ttll3 $^{--}$Ttll8 $8^{--}$sperm cells, their non-linear swimming pattern, clearly hampered their capacity to reach the site of fertilisation. To achieve progressive swimming, sperm require a symmetric flagellar beat, which $\mathrm{Ttll}^{-/-} \mathrm{Ttll}^{-/-}$sperm fail to generate. A higher beat asymmetry, as seen for $\mathrm{Ttll}^{-/-} \mathrm{Ttll}^{-/}$sperm, is known to result in an increased rotational velocity perpendicular to the translational velocity of the sperm cell $(20,29,30)$, and consequently induces the swimming along helical or circular paths. Even helical swim paths can become circular when sperm cells encounter a surface, thus impeding progressive movement (28). Thus, while Ttll3 $^{-/}$Ttll8 $^{-/}$sperm can advance, and eventually proceed to fertilisation, they are highly inefficient compared to wildtype sperm. It thus appears that the perturbed flagellar beat, which results in non-progressive swim paths, is most likely the reason for the observed subfertility of the ttll3 $^{-/-}$Ttll $^{-/-}$mice.

\section{Lack of glycylation leads to an altered distribution of axonemal dynein conformations}

To understand the molecular basis of the perturbed beat patterns of sperm flagella that lack glycylation, we determined native three-dimensional structures of axonemes in situ by plungefreezing swimming sperm and imaging their flagella using cryo-electron tomography. We then 
applied subtomogram averaging to the straight segments of the distal part of the flagella principal piece to generate high-resolution 3D electron density maps of the axonemal 96-nm repeat (31).

First, we observed that the absence of glycylation in ttll3 $^{-/-}$Ttll $^{-/-}$axonemes affected neither the assembly of the MTs, nor the overall macromolecular structure of the axonemes (Fig 5A,B). We also observed other axonemal features, such as a barrel-shaped structure between the radial spokes 1 and 2 and a protein structure linking the neck regions of radial spokes 2 and 3 (Fig 5A). These structures, which were not seen in axonemes of other species, were present in both wildtype and Ttll3 $^{--}$Ttll $^{-/-}$mouse sperm flagella. However, the difference map between the 96-nm repeat models of wildtype and $\mathrm{Ttll3}^{-/} \mathrm{Ttll}^{-/-}$axonemes revealed structural alterations in the $T t l l 3^{-/-}$Ttll $^{-/-}$axonemal dynein arms and their MT-binding domains on the B-tubule of each MT doublet (Fig 5C). The molecular identity of these barrel and linker structures remains unclear.

Axonemal dynein motors - the outer and inner dynein arms (ODAs and IDAs) - produce the force necessary to power flagellar motion and are organised in complexes that link together neighbouring axonemal MT doublets (32). Functionally, ODAs are thought to control the beat amplitude and frequency and IDAs the beat waveform (33). Consequently, loss or mislocalisation of specific axonemal dynein isoforms in a flagellum can cause alterations of the flagellar beat. However, this appeared not to be the case in $T t l l 3^{--} T_{t l l 8^{--}}$axonemes, because all ODA and IDA heavy chains were present in the 3D electron density maps (Fig 5A,C, 6A,B). Moreover, we confirmed that the two ODA heavy chains (DNAH17: $\beta$-heavy chain, DNAH8: $\gamma$-heavy chain; Fig S7A) and the IDA heavy and light chains (Fig S7B; DNAH2: IDA-f $\beta$-heavy chain, DNALI1: light intermediate chain) were present and correctly distributed along the entire

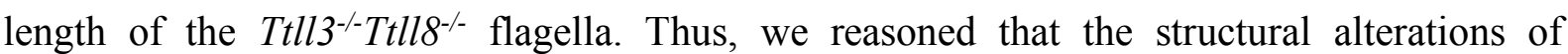


Ttll3 $^{--}$Ttll $8^{--}$axonemes that were highlighted by the difference map must have been of a more subtle nature.

Proper spatial and temporal coordination of dynein motor activities is crucial to drive flagellar motility. Experimental evidence from sea urchin sperm and Chlamydomonas flagella shows that in beating flagella, most ODAs adopt a pre-powerstroke conformation, while intermediate and post-powerstroke conformations are merely found on subsets of MTs in the bent regions of axonemes (34). Regardless of the nature of the mechanism that initiates the beating of the flagellum, which is still debated in the field, a spatially clustered distribution of active and inactive dynein motors, which correlates with the bending of the flagellum, is required for the propagation of the normal bending motion.

Our analysis of straight segments of active wildtype mouse sperm flagella showed that ODAs were predominantly in the pre-powerstroke conformation (Fig 6A; Movie S4A,B), indicating that the distribution of dynein conformations, previously observed in echinoderms and algae (34), is also conserved in mammals. In contrast, analysis of Ttll $^{-/-}$Ttll $8^{-/}$flagella showed dramatically altered structures of the $\beta$ - and $\gamma$-heavy chains of ODAs (Fig 6A; Movie S4A,B). The average of all Ttll3 $^{-/}$Ttll8 ${ }^{-/}$ODAs from straight axoneme segments showed poorly resolved heavy chains (Fig 6A), which is characteristic of flexible or conformationally heterogenous structures that disappear in subtomogram averages. This observation indicated defects in the conformational arrangement of the dynein heavy chains in $T t l l 3^{-/-}$Ttll $^{-/-}$axonemes, which we confirmed with a subtomogram classification approach. Only $26 \%$ of $T t l l 3^{-/} T_{t l l 8^{-/}}$ODAs exhibited the pre-powerstroke conformation, about $18 \%$ could not be classified, and the remaining $56 \%$ showed either the post-powerstroke state $(32 \%)$, or mixed conformations of opposing states of the $\beta$ - and $\gamma$-heavy chains, either as pre-post (19\%) or post-pre conformations (5\%) (Fig 6B,C; Movie S4C). Notably, the $\beta$-heavy chains were most affected by the absence 
of glycylation. The space distribution of the ODA conformations in the $T t l l 3^{-/-}$Ttll $^{-/}$flagellar segments was considerably altered as compared to wildtype flagella (Fig 6D), did not reveal a discernible pattern, and showed uncoordinated dynein activity. This altered conformational distribution of ODAs is presumably linked to an altered force generation profile that results in an aberrant beating phenotype.

Subtomogram averaging of $T t l l 3^{-/} T_{t l l 8^{-/}}$axonemes further revealed that IDAs also show an abnormal shift of their heavy chains towards the MT plus-end (Fig S8A), a conformation characteristic of immotile flagella (34). Subtomogram classification of IDAs from sperm flagella (Fig S8B-D) revealed that in wildtype, $42 \%$ of the IDAs were in pre-powerstroke, and $22 \%$ in a post-powerstroke conformation (Fig S8C,D). By contrast, only $20 \%$ of the Ttll3 $^{--}$Ttll $^{-/-}$IDAs were in pre-powerstroke and 45\% IDAs were in post-powerstroke conformation (Fig S8C,D). Among all IDAs analysed, IDA-f showed the most prominent structural and positional difference between wildtype and $T t l l 3^{-/} T t l l 8^{-/}$sperm flagella (Fig S8B). The conformational reconfiguration of IDA-f occurred concurrently with an opposing tilting motion of the nexin-dynein regulatory complex (N-DRC) and the radial-spoke heads. This movement was perpendicular to the MT axis and associated with a displacement of the next MT doublet (Movie S5). Similar movements have also been described for the nexindynein regulatory complex in Chlamydomonas axonemes (35), and the MT doublets in sea urchin sperm flagella (34).

\section{Mechanisms by which glycylation affects flagellar beat}

Our structural data show that the lack of glycylation in $T t l l 3^{-/} T_{t l l 8^{-/}}$sperm axonemes does not affect the binding and assembly of dyneins to A-tubules of the axonemal MT doublets (Fig 6A, S8A, Movie S4). Nevertheless, the lack of glycylation perturbed the coordination of motor activities. Thus, the affinity of the dynein MT-binding domain for the B-tubule may depend on 
tubulin glycylation, potentially changing the dynamics of the "re-loading" from the postpowerstroke into pre-powerstroke state. Indeed, in vitro single-molecule experiments have shown that other tubulin PTMs can alter certain aspects of the interactions between dynein motors and MTs $(36,37)$. Perturbations of dynein-MT interactions are expected to affect the flagellar beat characteristics, such as the amplitude and the frequency, which is what we observed for Ttll3 $^{--}$Ttll8 $^{--}$sperm (Fig 3C,D). Yet, it remains unclear why the flagellar beat of Ttll3 $^{-/-}$Ttll $^{-/-}$sperm features a higher mean curvature, and why the midpiece and the principal piece displayed different beat frequencies. The observed increased mean curvature of $T_{t l l 3^{-/}}$Ttll $^{-/-}$flagella could either be caused by a differential distribution of glycylated tubulin across different MT-doublets and the resulting effect of this asymmetry on motor activity, or be the effect of a generalised increase in motor activity on an inherently asymmetric axonemal structure. Whatever the origin, such asymmetry would result in bending in one preferred direction and axonemal twist, which has been suggested to control the switching point of flagellar oscillations (38). The outer dense fibres and the mitochondrial sheath, structures that are present in the flagellar midpiece, could prevent flagellar twist at this location (39). This would result in the observed arc-length dependent beat frequencies of the strongly bent Ttll $^{-/-}$Ttll $8^{-/-}$flagellum. In addition, the altered dynein activity we observed might result in local inhibition of MT sliding. Sliding inhibition, in turn, could result in secondary waves that emanate further up in the flagellum, as observed in flagella lacking glycylation (Movie S1, S2). The increased frequency and reduced amplitude of these waves would be consistent with findings in demembranated sperm (40).

Our observation that tubulin glycylation is essential for the coordination of the power-stroke cycle of axonemal dynein motors does not exclude the possibility that additional processes sensitive to glycylation contribute to the observed flagellar beat phenotype. For instance, proper flagellar beat also involves the central pair MTs that transmit mechanical signals via the radial 
spokes through the nexin-dynein regulatory complex, which control both IDAs and ODAs (41, 42). Indeed, central-pair MTs are also glycylated (43), and so we cannot exclude the possibility that the potential absence of glycylation on these MTs also contributes to a perturbed swimming pattern.

To verify if the lack of glycylation also affects the beating of other motile cilia in Ttll3 ${ }^{-/-}$Ttll $^{-/-}$ mice, we recorded ependymal cilia of brain ventricles in isolated brain slices. Cilia from both, wildtype and $\mathrm{Ttll}^{-/} \mathrm{Ttll}^{-/}$mice showed similar beat frequencies in three independent experiments (Movie S6), suggesting no major beat defects. However, ependymal cilia are shorter as compared to the sperm flagella, and might, similar to respiratory cilia in the trachea (44), contain different dynein heavy chains in their axonemes. Thus, the sensitivity of motile cilia to glycylation might be different from what we observed in sperm flagella.

While our data demonstrate a causative role of glycylation for the observed defects of Ttll3 $3^{--}$Ttll $^{-/}$sperm, other tubulin PTMs might also be involved. Earlier studies in Tetrahymena thermophila as well as in murine photoreceptors have revealed that absence of tubulin glycylation leads to an increase in tubulin polyglutamylation $(5,45,46)$. This is intuitive, because both PTMs use overlapping modification sites on tubulin (47). Accordingly, we also found an increase in tubulin polyglutamylation in $T t l l 3^{-/}$Ttll $^{-/-}$sperm (Fig S9). Nevertheless, it is rather unlikely that increased polyglutamylation participates in the observed defects in Ttll3${ }^{-}$Ttll $^{-/-}$sperm, given that the defects in mice lacking the deglutamylases CCP1 $(9,11)$ or CCP5 (48) are substantially different from those found in $\mathrm{Ttll3}^{-/-} \mathrm{Ttll}^{-/-}$mice.

\section{Conclusion}

Here we have elucidated the functional roles and molecular mechanisms of tubulin glycylation in ciliary and flagellar functions. Having generated a mouse model entirely lacking tubulin glycylation allowed us to reveal the importance of this PTM for mammalian sperm flagella. We 
demonstrated that the functional distribution of post- and pre-powerstroke conformations of axonemal dynein arms, which is an essential prerequisite for a physiological flagellar beat (33, $34,42)$, depends on the presence of tubulin glycylation. Absence of glycylation in flagella of Ttll3 $^{-/-}$Ttll $^{-/-}$sperm led to severe alteration of the symmetry, amplitude and frequency of the flagellar beat, which resulted in sperm failing to swim along a straight line. This, however, is a crucial requirement for sperm to reach the oocyte in the female reproductive tract. While Ttll3 $^{--}$Ttll8 $8^{--}$males were fertile, they showed smaller litter sizes and were revealed to be subfertile using in vitro fertilisation assays. Human sperm are more susceptible to deficiencies as compared to mouse sperm (49) and sperm motility defects such as asthenozoospermia account for almost $80 \%$ of the sperm defects observed in infertile men (50). Thus, our findings could have implications for human fertility.

Our cryo-electron tomography of the mammalian sperm flagellum uncovers the molecular mechanism by which glycylation supports flagellar beating, and in turn impacts sperm swimming and male fertility. Considering the massive evolutionary pressure on sperm fitness $(51,52)$, our findings might explain why glycylation has, with only a few exceptions, been maintained throughout evolution. Finally, our work implies that a perturbation of tubulin glycylation could potentially provide a molecular cause of male infertility in humans. 


\section{Materials and Methods:}

\section{$\underline{\text { Transgenic mice }}$}

\section{Generation of Ttll3 $^{-/}$and Ttll8 $8^{--}$mice}

The Ttll $^{-/-}$mice were generated in the lab as previously described (5) (Fig S1A). The Ttll $8^{\text {floxflox }}$ mouse was generated from the Ttll $8^{\operatorname{tm} l a(E U C O M M) W t s i}$ targeting vector obtained from EUCOMM consortium (IKMC Project \#83158), at the Institut Clinique de la Souris, Strasbourg, France. The targeting vector was constructed as follows: the $5^{\prime}-(4.7 \mathrm{~kb}) 3^{\prime}-(4.7 \mathrm{~kb})$ of Ttll8, LacZ and inter-loxP $(0.6 \mathrm{~kb})$ fragments were PCR-amplified and sequentially sub-cloned into an MCI proprietary vector containing the LoxP sites and a Neomycin resistance cassette flanked by the Flippase Recognition Target (FRT) sites (Fig S1B). The linearised construct was electroporated in 129S2/SvPas mouse embryonic stem (ES) cells. After selection, targeted clones were identified by PCR using external primers and further confirmed by Southern blot with 5' and 3' external probes. Two positive ES clones were injected into blastocysts, and derived male chimeras gave germline transmission. The excision of the neomycin-resistance cassette was performed in vivo by breeding the chimeras with a Flp deleter line (C57BL/6N genetic background FLP under ACTB promoter) to generate the Ttll $8^{\text {floxflox }}$ mice. To generate Ttll8-/ mice, we excised exon 2 by crossing Ttll8floxfllox mice with mice expressing cre-recombinase under the control of a PGK promoter (53).

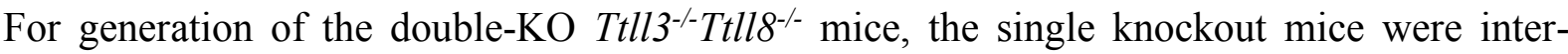
crossed to generate the heterozygotes $\mathrm{Ttll}^{+/-} \mathrm{Ttll8^{+/- }}$ which were later crossed between each other to generate the homozygote $T t l l 3^{-/}$Ttll $^{-/-}$mice.

All animals were backcrossed at least seven generations to the C57BL/6N animals.

\section{Mouse Genotyping}

DNA was extracted from ear fragments (collected during mouse identification) or tail fragments, using proteinase K (\#193504, MP Biomedicals). PCR-based genotyping was 
performed using primers listed in Fig S1C and protocols listed in Fig S1D. Examples of genotyping results for all transgenes and deletions are shown in Fig S1E.

\section{Mouse strain breeding}

Animal care and use for this study were performed in accordance with the recommendations of the European Community (2010/63/UE) for the care and use of laboratory animals. Experimental procedures were specifically approved by the ethics committee of the Institut Curie CEEA-IC \#118 (authorisation n04395.03 given by National Authority) in compliance with the international guidelines.

\section{$\underline{\text { Tissue histology }}$}

6-month old mice were anaesthetised by intraperitoneal application of Ketamine/Xlyazine (100 mg/kg body weight for Ketamine and $10 \mathrm{mg} / \mathrm{kg}$ body weight of Xylazine) and subjected to cardiac perfusion with phosphate-buffered saline (PBS; $\mathrm{pH}$ 7.4) containing 4\% paraformaldehyde (PFA). Post perfusion, the mice were dissected, and the tissues were postfixed with $4 \%$ PFA in PBS overnight at $4{ }^{\circ} \mathrm{C}$. The fixed tissues were washed and embedded in paraffin. 7- $\mu \mathrm{m}$ sections were obtained using a microtome (\#RM2245, Leica Biosystems). Sections were further analysed using immunocytochemistry or hematoxylin-eosin staining.

\section{Sperm isolation and Coomassie blue staining}

Sperm from 3-month-old male mice were stained with Coomassie blue staining solution as described earlier (48). Briefly, the mice were cervically dislocated, and the epididymides isolated and lacerated in $1 \mathrm{ml}$ PBS using 26G needles $(0.45 \times 13 \mathrm{~mm}$; \#613-5377, VWR) to allow the spermatozoa to swim out from the epididymides by incubating for $15 \mathrm{~min}$ at $37^{\circ} \mathrm{C}$. The sperm suspensions obtained from both wildtype and $T t l l 3^{-/-} T t l l 8^{-/-}$mice were fixed with $4 \%$ PFA, centrifuged at $500 \mathrm{~g}$ for $5 \mathrm{~min}$ at room temperature (RT), resuspended in $100 \mathrm{mM}$ ammonium acetate, and a smear was prepared with 50-70 $\mu 1$ of the sample on previously coated poly-L-lysine glass slides. Once the smear was dry, it was rehydrated and stained with $0.22 \%$ 
Coomassie blue (\#35050, Serva Electrophoresis $\mathrm{GmBH}$ ) for $2 \mathrm{~min}$ at RT. The slides were subsequently washed with water, mounted with Aquatex mounting medium (\#108562, Merck Millipore) and analysed.

\section{$\underline{\text { Culture of primary ependymal cells }}$}

Primary ependymal cells were cultured using the well-established protocol for isolation,

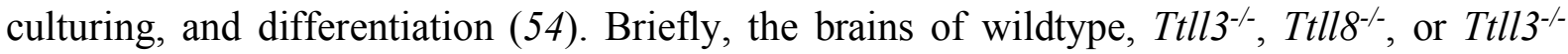
Ttll8 ${ }^{-}$PN0-PN2 mouse pups were isolated and dissociated, and the cells from the subventricular zone were isolated by dissociating the ventricles using papain for $1 \mathrm{~h}$ at $37^{\circ} \mathrm{C}$. The cells were plated at high density in DMEM containing $10 \%$ FBS in a $25-\mathrm{cm}^{2}$ flask precoated with poly-l-lysine (P4832; Sigma Aldrich). When cells reached confluency, the astroglial monolayer was re-plated onto precoated $12-\mathrm{mm}$ or 24-mm glass coverslips respectively in 24 -well or 6 -well plates at a density of $10^{4}$ cells $/ \mu 1$ and maintained in serum-free medium to enable differentiation.

\section{Dissection of lateral ventricles and whole-mount immunostaining}

To analyse the ependymal cilia in the brain ventricle by immunocytochemistry, whole-mount sections of the ventricles were obtained as per the protocol described earlier (54). Briefly, PN15

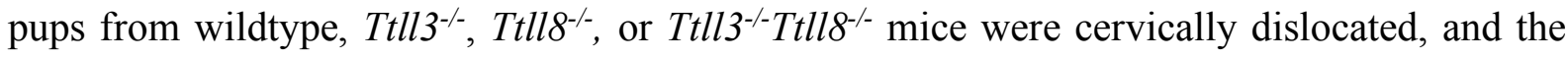
whole brain was retrieved by carefully peeling off the skull without disturbing the brain tissue using spring scissors and forceps. The brain was dissected in PBS using a micro knife under a stereomicroscope. During the entire dissection procedure, the tissue was held by inserting the forceps above the corpus callosum and into the thalamus to ensure the ventricle wall was not disturbed. Once the two cortical hemispheres were separated, the hippocampus was carefully removed, followed by the median wall. Then, to ensure better access to the antibodies, the thalamus, choroid plexus, and corpus callosum were separated from the ventricle. 
Once the lateral wall was obtained, it was permeabilised with $0.1 \%$ Triton X-100 in BRB80 (80 mM K-Pipes, pH 6.8, $5 \mathrm{mM} \mathrm{Na-EGTA,} 5 \mathrm{mM} \mathrm{MgCl}_{2}$ ) for $90 \mathrm{~s}$, followed by fixing with $4 \%$ PFA in BRB80 for 20 min at RT. The tissue was then carefully washed $3 \times$ with PBS, blocked for $1 \mathrm{~h}$ with saturation buffer (PBS containing 10\% FBS, 0.1\% Triton X-100), and stained with $200 \mu \mathrm{l}$ primary antibodies listed in Table S2 in a 2-ml Eppendorf tube at $4^{\circ} \mathrm{C}$ overnight. After $3 \times$ washing off the primary antibodies with PBS containing $0.1 \%$ Triton X-100, the tissues were stained with the respective secondary antibodies, as indicated in Table S2 for $1 \mathrm{~h}$ at RT. All the antibodies were diluted in saturation buffer. Nuclei were visualised by staining for 5 min with DAPI (0.02 $\mu \mathrm{g} / \mathrm{ml}$; \#D3571; Thermo Fisher Scientific).

After the staining, the tissues were further dissected prior to mounting. First, the cortex around and under the lateral wall was excised, taking care of the curvature and depth of the lateral wall. The rostral part of the wall was cut prior to the caudal part. The tissue was held using forceps inserted into the thalamus. Once the thalamus is finally removed, the tissue is transferred to a clean slide and mounted using ProLong Gold (\#P36934, Thermo Fisher Scientific), taking care that there is no excess pressure exerted on the tissue while mounting.

\section{Staining samples for immunofluorescence microscopy}

Immunocytochemistry of testes, kidney and trachea was carried out according to the established protocols (55). In brief, the tissue sections on the slides were hydrated with PBS, immersed in $10 \mathrm{mM} \mathrm{Na}$-citrate $\mathrm{pH} 6.0$, and boiled in a microwave for $7 \mathrm{~min}$ to enable antigen retrieval. The slides were subsequently cooled in ice for $1 \mathrm{~h}$, after which they were blocked with blocking buffer (PBS containing 10\% Normal Goat Serum (NGS), 0.3\% Triton X-100). Immunostaining of the different dyneins in spermatozoa was carried out by preparing smears of the wildtype and $\mathrm{Ttll}^{-/-} \mathrm{Ttll}^{-/-}$sperm on clean glass slides. The smears were fixed with $4 \%$ paraformaldehyde for $10 \mathrm{~min}$, followed by antigen retrieval and permeabilised with PBS containing $0.2 \%$ TritonX100. The smears were then blocked for $1 \mathrm{~h}$ at RT with buffer containing 1\% BSA. The slides 
were then stained with the different primary antibodies as listed in Table S2 overnight at $4{ }^{\circ} \mathrm{C}$ in a humid chamber. After washing the slides $4 \times 5$ min with PBS containing $0.1 \%$ Triton $X-$ 100 , the sections were stained with the respective secondary antibodies as listed in Table S2 for $1 \mathrm{~h}$ at RT. Most of the antibodies used were diluted in PBS containing 5\% NGS, 0.3\% Triton $\mathrm{X}-100$, except the dynein antibodies which were diluted in PBS containing $1 \%$ BSA, $0.02 \%$ Triton X-100. Nuclei were visualized by staining for 5 min with DAPI $(0.02 \mu \mathrm{g} / \mathrm{ml}$; \#D3571; Thermo Fisher Scientific). The slides were subsequently washed and mounted with ProLong Gold (\#P36934, Thermo Fisher Scientific) or DAPI Vectashield (\#H1200, Vector Laboratories).

Microscopy of histology, immunocytochemistry and immunofluorescence All immunofluorescence images apart from the whole-trachea section and sperm immunofluorescence (in Fig S2B and S7, respectively) were acquired using the Nikon Ti-E spinning disk inverted confocal laser microscope using either a $60 \times($ oil immersion; numerical aperture (NA) 1.40) or 100× (oil immersion; NA 1.49) objectives at RT. Images were acquired using the Prime95B sCMOS camera (Photometrics) and Leica MM AF imaging software. Multiple Z-stacks were acquired, and a maximum intensity projection was prepared in Fiji v2.1.0 (National Institute of Health) to generate the final image. Whole-trachea section were imaged using the Structured Illumination Microscope (Optigrid/Leica systems) with a $5 \times$ (NA 0.15) objective at RT, an ORCA-Flash4.0 camera (Hamamatsu), and Leica MM AF imaging software and processed using Fiji v2.1.0. For the histology, haematoxylin-eosin stained tissue sections were imaged in the Axio Imager.M2 microscope (Zeiss) using either a 10× (NA 0.3), $20 \times($ NA 0.5 ), or a $63 \times$ (water immersion; NA 1.2) objective. Multiple tiles of each tissue section were imaged. Tiles were subsequently stitched, background adjusted and processed using the ZEN v2.3 software (Zeiss). Sperm immunofluorescence was imaged using Zeiss Axiophot epifluorescence microscope with a $63 \times$ (oil immersion; NA 1.40) or 100× (oil 
immersion; NA 1.49) objective using a cooled charge-coupled device (CCD) camera (Hamamatsu) under identical instrument settings, with MetaMorph software (Molecular Devices).

\section{$\underline{\text { Immunoblot analyses }}$}

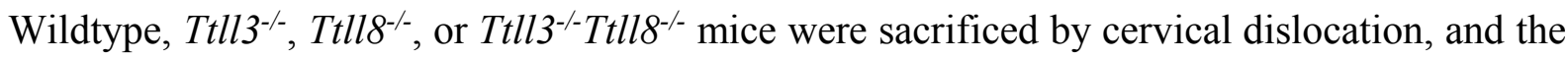
trachea were harvested in $2 \mathrm{ml}$ vials. The trachea were weighed and resuspended in lysis buffer (PBS pH 7.5, containing 25 mM HEPES, $200 \mathrm{mM} \mathrm{NaCl,} \mathrm{5 \%} \mathrm{glycerol,} 1 \mathrm{mM}$ DTT, 0.5\% NP40 and protease inhibitor cocktail) at $100 \mu \mathrm{l}$ per $100 \mathrm{mg}$ of the tissue and homogenized. The homogenate was then centrifuged at $10,000 \mathrm{~g}$ for $10 \mathrm{~min}$ at $4{ }^{\circ} \mathrm{C}$ and the supernatant collected. The epididymides from these mice were lacerated in $1 \mathrm{ml}$ PBS to allow the spermatozoa to swim out from the epididymites by incubating for $15 \mathrm{~min}$ at $37^{\circ} \mathrm{C}$. The sperm suspensions was centrifuged at $500 \mathrm{~g}$ for $5 \mathrm{~min}$ at $4^{\circ} \mathrm{C}$, after which the sperm pellets obtained were lysed in 100 $\mu \mathrm{l}$ of lysis buffer, centrifuged at $10,000 \mathrm{~g}$ for $10 \mathrm{~min}$ at $4^{\circ} \mathrm{C}$ and the supernatant collected. After estimating the total protein using the Pierce BCA protein estimation kit (\#23227; Life Technologies), 1-2 $\mu \mathrm{g}$ of the total protein was run on 10\% SDS-PAGE gels. A specific protocol for SDS-PAGE gels was used to separate $\alpha$ - and $\beta$-tubulin (56). Proteins were transferred to nitrocellulose membranes with Trans-Blot Turbo using transfer packs (\#1704159; Bio-Rad) and subjected to immunoblot analysis.

The ciliated cells, grown on 6-well plates were harvested at different time intervals, with $500 \mu 1$ of Laemmli buffer (400 mM Tris-HCl, pH 6.8 containing $450 \mathrm{mM}$ DTT, 10\% (wt/vol) SDS, $50 \%$ (vol/vol) glycerol, $\sim 0.006 \%$ (wt/vol) bromophenol blue), heated to $95^{\circ} \mathrm{C}$ for $10 \mathrm{~min}$ and equal protein was run on 10\% SDS-PAGE gels and analysed by immunoblot analysis.

For all immunoblots, the membranes were blocked overnight with PBS containing $0.1 \%$ Tween 20 (PBS-T) and 5\% non-fat milk and then incubated for $2 \mathrm{~h}$ at RT with the different primary antibodies as listed in Table S2. Antibodies were diluted in PBS-T containing 2.5\% 
non-fat milk. Membranes were washed four times with PBS-T and then incubated for $1.5 \mathrm{~h}$ with respective secondary antibodies conjugated to HRP, as listed in Table S2, diluted to 1:10,000 in PBS-T. After washing, antibody labelling was revealed using Clarity Western ECL detection reagent (\#1705061; Bio-rad).

Ependymal cilia beating analyses

\section{Image acquisition and generation of videos}

Brain sections from PN20-PN30 wildtype and $T t l l 3^{-/}$Ttll $^{-/-}$mice were collected and imaged as described in (57). Briefly, the dissected brains were sectioned into $\sim 200 \mu \mathrm{m}$ sagittal sections in Leibovitz L15 medium. The sections were then placed in $100 \mu 1$ medium on a glass slide and covered with a $24 \times 60 \mathrm{~mm}$ glass coverslip no. 1 by gently sealing it with vacuum grease. The sections were immediately imaged using the CLSM-SP5 confocal light microscope (Leica Biosystems) equipped a resonance scanner for high-speed imaging, using a 60× (oil immersion; NA 1.4) objective at $37^{\circ} \mathrm{C}$. The sections were imaged at $64 \times 48$ pixels $(15 \times$ zoom $)$ at 100 frames per second (FPS) for 3-5 s using the LSM software, to obtain beating cilia with minimal tissue background. The time series obtained were exported as .avi videos, which were adjusted for their brightness and contrast using Fiji v2.1.0. For better visualisation of the curvature of the ciliary beat, the videos were slowed down 20 -fold, to run at 5 FPS

\section{Cilia beat frequency analysis}

To analyse the ependymal cilia beat frequency (CBF), the videos obtained for both the wildtype and $\mathrm{Ttll}^{-/-} \mathrm{Ttll}^{---}$mice were analysed using the CiliaFA plugin developed for ImageJ (58) that allowed fast, automated beat frequency analysis minimizing manual errors. The software works on extracting the pixel intensities, coupled with fast Fourier transformation in Microsoft Excel to provide the CBF. 256 frames of each video obtained from the wildtype or Ttll3 ${ }^{-/}$Ttll8 $^{-/}$brain sections were analysed in CiliaFA. The CBF obtained were tabulated and quantified using GraphPad prism version 8 . 


\section{$\underline{\text { Sperm motility analyses }}$}

\section{Sperm preparation for motility and holographic analyses of flagellar beat}

Mouse sperm were collected by cutting off the cauda epididymites followed by a swim-out process in modified TYH medium (10 mM HEPES pH 7.4, $135 \mathrm{mM} \mathrm{NaCl}, 4.8 \mathrm{mM} \mathrm{KCl,} 2 \mathrm{mM}$ $\mathrm{CaCl}_{2}, 1.2 \mathrm{mM} \mathrm{K \textrm {KH } _ { 2 } \mathrm { PO } _ { 4 } , 1 \mathrm { mM } \mathrm { MgSO }}$, $5.6 \mathrm{mM}$ glucose; $0.5 \mathrm{mM}$ Na-pyruvate, $10 \mathrm{mM}$ L-lactate) for $30 \mathrm{~min}$ at $37^{\circ} \mathrm{C}$. Sperm were collected carefully without touching oil and tissue fragments.

\section{Analysis of sperm motility and flagellar beating}

Freely swimming and tethered sperm were studied in TYH medium containing $3 \mathrm{mg} / \mathrm{ml}$ and $0.3 \mathrm{mg} / \mathrm{ml} \mathrm{BSA}$, respectively. Images were recorded using a high-speed camera (pco.dimax; PCO AG) and an inverted microscope (IX 71; Olympus). The temperature of the microscope was adjusted to $37^{\circ} \mathrm{C}$ using an incubator (Life Imaging Services, Switzerland). Wide-field images were recorded at 50 FPS using a $4 \times$ objective (UPLanFLN; Olympus) and custom-made observation chambers with a $100-\mu \mathrm{m}$ depth. Illumination was achieved using a 660-nm LED. Dark-field and holographic recordings were achieved using a 10× objective (UPLSAPO10X; Olympus) and an additional 1.6× magnifying lens.

Dark-field images were recorded at 250 FPS using a M730L4 LED light source. For analysis, images were processed by a Gaussian blur (sigma: $0.5 \mathrm{px}$ ) and the Subtract Background method (radius: 5 px) in ImageJ. Next, the flagellar analysis was performed using SpermQ (20). Positive curvature refers to bending in the direction of the hook-shaped sperm head. The peak frequency was determined from the Fast-Fourier transform of the curvature values. Individual values for all the analyses are provided in Table S3.

3D holographic images were recorded at 500 or 1,000 FPS in observation chambers with a $150-\mu \mathrm{m}$ depth. Coherent illumination was produced by a $510-\mathrm{nm}$ laser (LDH-D-C serials, PicoQuant $\mathrm{GmbH}$ ) and the corresponding controller (Sepia II Multichannel Processor, 
PicoQuant $\mathrm{GmbH}$ ). Holograms were analysed as previously described (25). In brief: The background was calculated by averaging all the holograms from each recording, and a background-free hologram was obtained by dividing the original hologram by the background. The Rayleigh-Sommerfeld back-propagation method (59) was used to numerically re-focus each background-free hologram, resulting in a re-focused stack. A filter based on the Gouy phase anomaly (60) was applied on the re-focused stack, and the 3D coordinates of the sperm flagellum were extracted by localising the brightest shape in the refocused stack. The head $\mathrm{x}$ and y coordinates were estimated by calculating the centre of mass of the scattering pattern from the sperm head. To determine robustly the $\mathrm{z}$ head coordinates, we fitted a plane to the basal end of the flagellum (about $11 \mu \mathrm{m}$ from the head centre). The head $\mathrm{z}$ coordinate was obtained by substituting the head $\mathrm{x}$ and $\mathrm{y}$ coordinates into the plane equation. All the analysis was done in the open-source software ImageJ combined with custom-made Java plugins.

3D trajectories predicted from flagellar beat patterns were computed using resistive-force theory as previously described $(25,29)$ using the $2 \mathrm{D}$ flagellar beat pattern from tethered sperm (Fig 3A) and assuming a constant $z=z(s)$ component characterised by an arc of curvature $\kappa_{\mathrm{z}}=$ $5 \cdot 10^{-3} \mu \mathrm{m}^{-1}$ along the full flagellar arc length $s$.

\section{Generating colour-coded projections}

Colour-coded videos and projections were generated using custom ImageJ macros. The macros and code are open source and freely available at https://github.com/hansenjn/ColorStackByTimeAndProject.

\section{Computer-assisted sperm analyses (CASA)}

Sperm motility was assessed by computer-assisted sperm analysis (CASA) using the CEROS II apparatus (Hamilton Thorne, Beverly, MA USA). Briefly, mouse sperm cells expelled from the cauda epididymis were recovered in M2 medium (Sigma-Aldrich, Saint-Louis, MO, USA) and incubated for $10 \mathrm{~min}$ at $37^{\circ} \mathrm{C}$ in $5 \% \mathrm{CO}_{2}$. The suspension was collected, and total sperm 
count determined. At least 500 sperm cells per sample were analysed in $20 \mu \mathrm{m}$ chambers (Leja Products B.V., Netherlands) with a Zeiss AX10 Lab. A1 microscope (10× objective) and using HT CASAII software.

The settings were as follows: acquisition rate, $60 \mathrm{~Hz}$; number of frames, 45; minimum head brightness, 175; minimum tail brightness, 80 ; minimum head size, $10 \mu \mathrm{m}^{2}$; minimum elongation gate, 1\%; maximum elongation gate, 100\%; objective magnification factor, 1.2.

The principal motility parameters measured were: curvilinear velocity (VCL), average path velocity (VAP), straight-line velocity (VSL), beat/cross frequency (BCF), amplitude of lateral head displacement (ALH). Progressive sperm cells were characterized by average path velocity $(\mathrm{VAP})>45 \mu \mathrm{m} / \mathrm{s}$ and straightness $(\mathrm{STR}=\mathrm{VSL} / \mathrm{VAP})>45 \%$, respectively.

\section{$\underline{\text { Sperm viability analyses }}$}

Sperm viability was assessed by eosine-nigrosin staining as previously described (61). Briefly, $10 \mu 1$ of sperm suspension was mixed with $20 \mu 1$ of Eosin and $30 \mu 1$ nigrosin, and a $20-\mu 1$ droplet was smeared onto a clean glass slide. After drying, a minimum of 200 cells per genotype were counted.

$\underline{\text { In-vitro fertilization analyses }}$

\section{Preparation of oocytes}

C75B6L/N female mice (6- to 8-week-old, Janvier Labs, France) were super-ovulated with 5 IU of pregnant mare serum gonadotropin (PMSG) and 5 IU human chorionic gonadotropin (hCG; Intervet, France) $48 \mathrm{~h}$ apart. About $14 \mathrm{~h}$ after hCG injection, the animals were sacrificed by cervical dislocation. Cumulus oophorus were collected by tearing the ampulla wall of the oviduct, placed in Ferticult medium (\#FECU100; FertiPro N.V, Belgium) supplemented with 3\% BSA (\#A3311; Sigma-Aldrich). To remove the zona pellucida (ZP), cumulus cells were removed by a brief exposure to hyaluronidase IV-S (1 mg/ml; \#H3884, Sigma-Aldrich) and ZP was then dissolved with acidic Tyrode's (AT) solution, pH 2.5 (\#T1788; Sigma-Aldrich) under 
visual monitoring. The zona-free eggs were rapidly washed five times with Ferticult medium and kept at $37^{\circ} \mathrm{C}$ under $5 \% \mathrm{CO}_{2}$ for $2-3 \mathrm{~h}$ to recover their fertilisation ability.

\section{Capacitated sperm preparation}

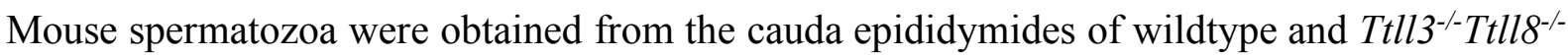
male mice (8- to 10 -week-old) and capacitated at $37^{\circ} \mathrm{C}$ under $5 \% \mathrm{CO}_{2}$ for 90 min in a $500-\mu 1$ drop Ferticult medium supplemented with 3\% BSA, under mineral oil. The total sperm count was obtained, and $10^{5}$ sperm per $\mathrm{ml}$ from either wildtype or $T t l l 3^{-/} T t l l 8^{-/-}$mice were used for each experiment.

\section{$\underline{\text { In vitro fertilisation }}$}

Zona-free eggs were inseminated with capacitated spermatozoa for $3 \mathrm{~h}$ in a $100-\mu 1$ drop of Ferticult medium 3\% BSA at a final concentration of $10^{5} / \mathrm{ml}$. Then, they were washed and directly mounted in Vectashield/DAPI (Vector laboratories, CA, USA) for observation under UV light (Nikon Eclipse E600 microscope). Only oocytes showing at least one fluorescent decondensed sperm head within their cytoplasm were considered fertilised. To assess the fertilization index (FI), the number of decondensed sperm heads per oocyte was counted.

\section{Cryo-EM analyses}

Cauda epididymides were extracted, lacerated in PBS and kept for $10 \mathrm{~min}$ at $37^{\circ} \mathrm{C}$. Swimming sperm cells were collected and rapidly plunge-frozen on Quantifoil Holey Carbon grids using a Leica Automatic Plunge Freezer EM GP. Samples were imaged with a FEI Titan Halo (Thermo Fisher Scientific) operated at $300 \mathrm{keV}$ using a Gatan energy filter and a Gatan K2 Summit direct electron detector. SerialEM software was used to operate the microscope during acquisition of tomographic tilt series. Tomographic series were acquired at angles from $-26^{\circ}$ to $40^{\circ}$ and $-28^{\circ}$ to $-40^{\circ}$ using a $2^{\circ}$ tilt increments. Each image was acquired in dose fractionation mode with about $2 \mathrm{e} / \AA^{2}$ total electron dose. Image frames was further processed with K2Align software (62) to generate each image of a tilt series. Generated tilt series were processed with 
etomo from IMOD software package (63) to reconstruct tomograms by weighted backprojection using fiducial-based aligned, CTF corrected, dose-weighted filtered image stacks (64). Tomograms were further filtered using non-linear anisotropic diffusion (63) prior to tomographic inspection and annotation. We chose straight segments of the sperm tails for our analyses to optimise the conditions for the tomographic reconstruction. The positions of the 96$\mathrm{nm}$ axonemal repeats and the associated ODAs within unfiltered tomograms were modelled using 3dmod. Initially, 983 96-nm repeats and 3166 ODA particles were extracted for wildtype while for ttll3 $^{-/-}$Ttll $^{-/} 617$ 96-nm repeats and 3656 ODA particles were extracted. All particles from each MT doublet were manually aligned to a common reference prior to fine automatic alignments and subtomogram averaging using PEET (65). For the final averages of the 96-nm repeat, 900 wildtype and $540 \mathrm{Ttll}^{-/-} \mathrm{Ttll}^{-/-}$particles were used (Fig S10). For the ODA related analysis, only particles from microtubule doublet pairs showing no mechanical disruption were included for further conformational analysis. The number of ODA particles used in this analysis was 2691 for wildtype and 3656 for $T t l l 3^{-/} T_{t l l 8^{-/}}$. Each ODA particle was classified according to the combined result of principal component analysis and k-means clustering (65) of particle regions containing either $\beta$ - or $\gamma$-heavy chains. IDAs were classified with principal component analysis of independently aligned wildtype and $T t l l 3^{--} T_{t l l 8^{-/}}$sperm flagella. This allowed their classification into pre- and post-powerstroke configurations. $3 \mathrm{dmod}$ was used to inspect the resulting averages and generate sliced views while further 3D rendering and structure fitting for clearer visualisation was carried out using Chimera (66). For the complete cryo-analysis, sperm samples from one wildtype mouse were used, while in the $T t l l 3^{-/-}$Ttll $^{-/}$case, the samples used came from two different mice.

\section{$\underline{\text { Statistical analyses }}$}

All the quantifications are listed in Table S1 
For $2 D$ and $3 D$ motility analyses: To determine the swimming patterns, $\sim 250$ sperm from 6 different wildtype and 5 different $T t l l 3^{-/} T t l l 8^{-/-}$mice were analysed.

For all the flagellar beat parameter analyses, 76 spermatozoa from 6 different wildtype mice and 58 spermatozoa from 4 different ttll3 $^{-/-}$Ttll $^{-/-}$mice were analysed.

For Ependymal cilia beat frequency analyses: To determine the CBF, sections from 3 individual wildtype or $T t l l 3^{--}$Ttll $^{-/-}$mice were analysed. From each section, 13-26 individual cells were imaged for their ciliary beat and CBF determined using CiliaFA plugin for ImageJ. Scatter plots from each experiment were plotted using GraphPad Prism version 8. The lines indicate the mean $( \pm$ SEM). P-value was calculated by unpaired student's t-test.

For CASA analyses: To determine the motility parameters, sperm from 5 wildtype and 6 Ttll3${ }^{-}$Ttll $^{-/-}$mice were analysed. The graphs for each of the individual motility parameters were plotted using GraphPad Prism version 8. Mean values of individual experiments were plotted as bar graphs with standard error of mean $( \pm$ SEM) between individual experiments. P-values were calculated by unpaired student's t-test.

For in vitro fertilisation assay: To analyse the fertilisation index of the sperm with in vitro fertilisation tests, the experiments were performed five times, using five individual wildtype and Ttll $^{-/-}$Ttll $^{-/-}$mice. The fertilisation index is plotted as a ratio of the total number of fused sperm to the total number of oocytes used per experiment. The graphs were plotted using GraphPad Prism version 8. Mean values of individual experiments were plotted in bar graphs with standard error of mean $( \pm \mathrm{SEM})$ between individual experiments. P-values were calculated by unpaired student's t-test.

\section{Image and video processing}

Immunofluorescence and histology images were treated with Adobe Photoshop. Intensities were adjusted uniquely in a linear manner, and no additional image treatments were performed. 
For mounting the composite videos, the still panels were prepared in Adobe Illustrator and then subsequently mounted onto the individual video compositions in Adobe After Effects. Each composition was rendered as a QuickTime movie with Adobe Media Encoder and exported as $1280 \times 720$ pixel videos with QuickTime Player v10.4. 


\section{References:}

1. C. Janke, M. M. Magiera, The tubulin code and its role in controlling microtubule properties and functions. Nat Rev Mol Cell Biol 21, 307 (2020).

2. M. H. Bré et al., Axonemal tubulin polyglycylation probed with two monoclonal antibodies: widespread evolutionary distribution, appearance during spermatozoan maturation and possible function in motility. J Cell Sci 109, 727 (1996).

3. K. Rogowski et al., Evolutionary divergence of enzymatic mechanisms for posttranslational polyglycylation. Cell 137, 1076 (2009).

4. M. Bosch Grau et al., Tubulin glycylases and glutamylases have distinct functions in stabilization and motility of ependymal cilia. J Cell Biol 202, 441 (2013).

5. M. Bosch Grau et al., Alterations in the balance of tubulin glycylation and glutamylation in photoreceptors leads to retinal degeneration. J Cell Sci 130, 938 (2017).

6. C. Rocha et al., Tubulin glycylases are required for primary cilia, control of cell proliferation and tumor development in colon. EMBO J 33, 2247 (2014).

7. S. Gadadhar et al., Tubulin glycylation controls primary cilia length. J Cell Biol 216, 2701 (2017).

8. A. Konno et al., Ttll9-/- mice sperm flagella show shortening of doublet 7, reduction of doublet 5 polyglutamylation and a stall in beating. J Cell Sci 129, 2757 (2016).

9. R. J. Mullen, E. M. Eicher, R. L. Sidman, Purkinje cell degeneration, a new neurological mutation in the mouse. Proc Natl Acad Sci U S A 73, 208 (1976).

10. P. Vogel, G. Hansen, G. Fontenot, R. Read, Tubulin tyrosine ligase-like 1 deficiency results in chronic rhinosinusitis and abnormal development of spermatid flagella in mice. Vet Pathol 47, 703 (2010).

11. H.-Y. Wu, P. Wei, J. I. Morgan, Role of cytosolic carboxypeptidase 5 in neuronal survival and spermatogenesis. Sci Rep 7, 41428 (2017).

12. C. Mencarelli, P. Lupetti, R. Dallai, New insights into the cell biology of insect axonemes. Int Rev Cell Mol Biol 268, 95 (2008).

13. J. F. Reiter, M. R. Leroux, Genes and molecular pathways underpinning ciliopathies. Nat Rev Mol Cell Biol 18, 533 (2017).

14. M. A. El-Brolosy, D. Y. R. Stainier, Genetic compensation: A phenomenon in search of mechanisms. PLoS Genet 13, e1006780 (2017).

15. M. A. El-Brolosy et al., Genetic compensation triggered by mutant mRNA degradation. Nature 568, 193 (2019).

16. R. Talevi, R. Gualtieri, In vivo versus in vitro fertilization. Eur J Obstet Gynecol Reprod Biol 115 Suppl 1, S68 (2004).

17. H. H. Pusch, The importance of sperm motility for the fertilization of human oocytes in vivo and in vitro. Andrologia 19, 514 (1987).

18. R. P. Amann, D. Waberski, Computer-assisted sperm analysis (CASA): capabilities and potential developments. Theriogenology 81, 5 (2014).

19. Z. Heidary, K. Saliminejad, M. Zaki-Dizaji, H. R. Khorram Khorshid, Genetic aspects of idiopathic asthenozoospermia as a cause of male infertility. Hum Fertil (Camb) 23, 83 (2020).

20. J. N. Hansen, S. Rassmann, J. F. Jikeli, D. Wachten, SpermQ - A simple analysis software to comprehensively study flagellar beating and sperm steering. Cells $\mathbf{8}$, 10.3390/cells8010010 (2018). 
21. S. T. Mortimer, D. Mortimer, Kinematics of human spermatozoa incubated under capacitating conditions. J Androl 11, 195 (1990).

22. J. M. Neill, P. Olds-Clarke, A computer-assisted assay for mouse sperm hyperactivation demonstrates that bicarbonate but not bovine serum albumin is required. Gamete Res $\mathbf{1 8}$, 121 (1987).

23. B. Marquez, G. Ignotz, S. S. Suarez, Contributions of extracellular and intracellular Ca2+ to regulation of sperm motility: Release of intracellular stores can hyperactivate CatSper1 and CatSper2 null sperm. Dev Biol 303, 214 (2007).

24. T.-W. Su et al., Sperm trajectories form chiral ribbons. Sci Rep 3, 1664 (2013).

25. J. F. Jikeli et al., Sperm navigation along helical paths in 3D chemoattractant landscapes. Nat Commun 6, 7985 (2015).

26. R. L. Miller, in Biology of Fertilization, C. B. Metz, A. Monroy, Eds. (Academic Press, 1985), pp. 275-337.

27. T.-W. Su, L. Xue, A. Ozcan, High-throughput lensfree 3D tracking of human sperms reveals rare statistics of helical trajectories. Proc Natl Acad Sci U S A 109, 16018 (2012).

28. J. Elgeti, U. B. Kaupp, G. Gompper, Hydrodynamics of sperm cells near surfaces. Biophys J 99, 1018 (2010).

29. A. Gong et al., The steering gaits of sperm. Philos Trans R Soc Lond B Biol Sci 375, 20190149 (2020).

30. H. C. Crenshaw, A new look at locomotion in microorganisms: Rotating and translating. Amer Zool 36, 608 (1996).

31. D. Nicastro et al., The molecular architecture of axonemes revealed by cryoelectron tomography. Science 313, 944 (2006).

32. R. Viswanadha, W. S. Sale, M. E. Porter, Ciliary motility: Regulation of axonemal dynein motors. Cold Spring Harb Perspect Biol 9, 10.1101/cshperspect.a018325 (2017).

33. M. E. Porter, W. S. Sale, The $9+2$ axoneme anchors multiple inner arm dyneins and a network of kinases and phosphatases that control motility. J Cell Biol 151, F37 (2000).

34. J. Lin, D. Nicastro, Asymmetric distribution and spatial switching of dynein activity generates ciliary motility. Science 360, eaar1968 (2018).

35. L. Gui et al., Scaffold subunits support associated subunit assembly in the Chlamydomonas ciliary nexin-dynein regulatory complex. Proc Natl Acad Sci U S A 116, 23152 (2019).

36. J. D. Alper, M. Tovar, J. Howard, Displacement-weighted velocity analysis of gliding assays reveals that Chlamydomonas axonemal dynein preferentially moves conspecific microtubules. Biophys J 104, 1989 (2013).

37. R. J. McKenney, W. Huynh, R. D. Vale, M. Sirajuddin, Tyrosination of alpha-tubulin controls the initiation of processive dynein-dynactin motility. EMBO J 35, 1175 (2016).

38. P. Sartori, V. F. Geyer, J. Howard, F. Jülicher, Curvature regulation of the ciliary beat through axonemal twist. Phys Rev E 94, 042426 (2016).

39. C. B. Lindemann, K. A. Lesich, Functional anatomy of the mammalian sperm flagellum. Cytoskeleton (Hoboken) 73, 652 (2016).

40. M. Fujimura, M. Okuno, Requirement of the fixed end for spontaneous beating in flagella. J Exp Biol 209, 1336 (2006).

41. E. F. Smith, P. Yang, The radial spokes and central apparatus: mechano-chemical transducers that regulate flagellar motility. Cell Motil Cytoskeleton 57, 8 (2004).

42. T. Kubo, Y. Hou, D. A. Cochran, G. B. Witman, T. Oda, A microtubule-dynein tethering complex regulates the axonemal inner dynein f(I1). Mol Biol Cell 29, 1060 (2018). 
43. R. Orbach, J. Howard, The dynamic and structural properties of axonemal tubulins support the high length stability of cilia. Nat Commun 10, 1838 (2019).

44. T. Ishikawa, Axoneme structure from motile cilia. Cold Spring Harb Perspect Biol 9 , 10.1101/cshperspect.a028076 (2017).

45. D. Wloga et al., TTLL3 Is a tubulin glycine ligase that regulates the assembly of cilia. Dev Cell 16, 867 (2009).

46. D. Wloga et al., Hyperglutamylation of tubulin can either stabilize or destabilize microtubules in the same cell. Eukaryot Cell 9, 184 (2010).

47. C. Janke, The tubulin code: Molecular components, readout mechanisms, and functions. J Cell Biol 206, 461 (2014).

48. T. Giordano et al., Loss of the deglutamylase CCP5 perturbs multiple steps of spermatogenesis and leads to male infertility. J Cell Sci 132, 10.1242/jcs.226951 (2019).

49. Z.-E. Kherraf et al., SPINK2 deficiency causes infertility by inducing sperm defects in heterozygotes and azoospermia in homozygotes. EMBO Mol Med 9, 1132 (2017).

50. S. M. Curi et al., Asthenozoospermia: analysis of a large population. Arch Androl 49, 343 (2003).

51. T. R. Birkhead, D. J. Hosken, S. Pitnick, Sperm Biology: An Evolutionary Perspective. T. R. Birkhead, D. J. Hosken, S. Pitnick, Eds., Sperm Biology (Academic Press, London, 2009), pp. vii-viii.

52. L. Alvarez, The tailored sperm cell. J Plant Res 130, 455 (2017).

53. Y. Lallemand, V. Luria, R. Haffner-Krausz, P. Lonai, Maternally expressed PGK-Cre transgene as a tool for early and uniform activation of the Cre site-specific recombinase. Transgenic Res 7, 105 (1998).

54. N. Delgehyr et al., Ependymal cell differentiation, from monociliated to multiciliated cells. Methods Cell Biol 127, 19 (2015).

55. S. M. Q. Hussaini et al., Heat-induced antigen retrieval: an effective method to detect and identify progenitor cell types during adult hippocampal neurogenesis. $J$ Vis Exp 78, e50769 (2013).

56. M. M. Magiera, C. Janke, in Methods Cell Biol, J. J. Correia, L. Wilson, Eds. (Academic Press, Burlington, 2013), vol. 115, pp. 247-67.

57. A. J. Al Omran, H. C. Saternos, T. Liu, S. M. Nauli, W. A. AbouAlaiwi, Live imaging of the ependymal cilia in the lateral ventricles of the mouse brain. J Vis Exp 100, e52853 (2015).

58. C. M. Smith et al., ciliaFA: a research tool for automated, high-throughput measurement of ciliary beat frequency using freely available software. Cilia 1, 14 (2012).

59. S.-H. Lee, D. G. Grier, Holographic microscopy of holographically trapped threedimensional structures. Optics Express 15, 1505 (2007).

60. L. Wilson, R. Zhang, 3D Localization of weak scatterers in digital holographic microscopy using Rayleigh-Sommerfeld back-propagation. Optics Express 20, 16735 (2012).

61. L. Bjorndahl, I. Soderlund, U. Kvist, Evaluation of the one-step eosin-nigrosin staining technique for human sperm vitality assessment. Hum Reprod 18, 813 (2003).

62. X. Li et al., Electron counting and beam-induced motion correction enable near-atomicresolution single-particle cryo-EM. Nat Methods 10, 584 (2013).

63. J. R. Kremer, D. N. Mastronarde, J. R. McIntosh, Computer visualization of threedimensional image data using IMOD. J Struct Biol 116, 71 (1996). 
64. Q. Xiong, M. K. Morphew, C. L. Schwartz, A. H. Hoenger, D. N. Mastronarde, CTF determination and correction for low dose tomographic tilt series. J Struct Biol 168, 378 (2009).

65. J. M. Heumann, A. Hoenger, D. N. Mastronarde, Clustering and variance maps for cryoelectron tomography using wedge-masked differences. J Struct Biol 175, 288 (2011).

66. E. F. Pettersen et al., UCSF Chimera - a visualization system for exploratory research and analysis. J Comput Chem 25, 1605 (2004). 


\section{Acknowledgements:}

We would like to thank C. Arnoult (University of Grenoble, France), S. Bodakuntla, M.M. Magiera (Institut Curie), E. Feraille (University of Geneva, Switzerland), T. Stünker, J. Wistuba (University Clinics Münster, Germany), D. Wachten (University of Bonn, Germany) for critical analysis and insightful discussions. We would also like to thank N. Spassky and N. Delgehyr (Ecole Normale Superieur, Paris) for technical assistance with the mouse primary ependymal cell culture and mouse ventricle whole-mount staining experiments. We acknowledge the Mouse Clinical Institute (PHENOMIN-Institut Clinique de la Souris, MCI/ICS) for generating the transgenic Ttll8 mice in the Genetic Engineering and Model Validation Department by M.C. Birling with funds from the CNRS. We thank J. Helppi (Biomedical Services of Max Planck Institute for Molecular Cell Biology and Genetics (MPI-CBG)) for managing the genetically modified mice and for helping with tissue dissection. We thank the Electron Microscopy facility of the MPI-CBG for technical support. We further thank C. Alberti, E. Belloir, F. Bertrand, V. Dangles-Marie, I. Grandjean, H. Hermange, C. Caspersen, M.M. Magiera, S. Bodakuntla, N.L. J. Nguyen, C. Rollin, C. Serieyssol, A. Thadal, L. Vaslin (Institut Curie) for technical assistance. We are grateful to M.-N. Soler, C. Lovo and L. Besse from the imaging platform PICT-IBiSA@Orsay (Institut Curie, Orsay) for technical assistance in the use of the Leica Structured Illumination Microscope (Optigrid) and the Nikon Eclipse Ti-E inverted microscope systems. We also thank B. Friedrich for his support using resistive-force theory.

\section{Funding:}

This work was supported by the ANR-10-IDEX-0001-02, the LabEx Cell(n)Scale ANR-11LBX-0038. CJ is supported by the Institut Curie, the French National Research Agency (ANR) awards ANR-12-BSV2-0007 and ANR-17-CE13-0021, the Institut National du Cancer (INCA) grant 2014-PL BIO-11-ICR-1, and the Fondation pour la Recherche Médicale (FRM) grant DEQ20170336756. SG received a 1-year postdoctoral fellowship from LabEx Cell(n)Scale 
ANR-11-LBX-0038. GP, GAV, and AK are supported by the Max Planck Society and the European Research Council (ERC) under the European Union's Horizon 2020 research and innovation program (grant agreement No. 819826) to GP. JNH is holding a PhD Fellowship by the Boehringer Ingelheim Fonds. The work of LA was supported by the Deutsche Forschungsgemeinschaft via the priority programme SPP 1726 'Microswimmers'. MW and AT are supported by the French National Research Agency (ANR) awards DIVERCIL-17-CE130023; ANR FLAGELOME 19-CE17-0014.

\section{Author Contributions:}

Conceptualisation: S. Gadadhar, L. Alvarez, G. Pigino, C. Janke.

\section{Methodology, analyses and validation:}

Mouse generation, genotyping, ependymal cell culture, immunoblot, immunocytochemistry, histological analyses: S. Gadadhar, S. Leboucher, M. Whitfield, C. Janke.

CASA and in vitro fertility analyses: A. Touré, M. Whitfield, A. Ziyyat, C. Ialy-Radio, S. Gadadhar, C. Janke.

2D motility and 3D in-line holography analyses: J.N. Hansen, A. Gong, L. Alvarez.

Computation of ideal trajectories using resistive-force theory: A. Gong, L. Alvarez.

Sample preparation for cryo-electron tomography: G. Alvarez Viar, A. Kostarev, S. Gadadhar. Cryo-electron tomography, subtomogram averaging, particle classification, 3D model rendering, and interpretation: G. Alvarez Viar, A. Kostarev, G. Pigino.

Writing: original draft: S. Gadadhar, C. Janke; review \& editing: S. Gadadhar, G. Alvarez Viar, J.N. Hansen, A. Gong, A. Touré, A. Ziyyat, L. Alvarez, G. Pigino, C. Janke.

Visualisation: S. Gadadhar, G. Alvarez Viar, J.N. Hansen, A. Gong, A. Kostarev, A. Touré, M. Whitfield, A. Ziyyat, L. Alvarez, G. Pigino, C. Janke.

Supervision: L. Alvarez, G. Pigino, A. Touré, C. Janke. 
Project administration: C. Janke.

Funding acquisition: S. Gadadhar, L. Alvarez, G. Pigino, A. Touré, C. Janke.

\section{Conflict of interest:}

The authors declare no competing financial interests.

\section{Data and materials availability}

The 3D averaged structures of the 96-nm repeats of the wildtype and TTLL $3^{-/} T T L L 8^{-/-}$sperm flagella, and all major classes of the ODAs and IDAs have been deposited in the Electron Microscopy Data Bank (EMDB) under ID codes EMD-11942 (pre-pre-powerstroke conformation of the ODAs), EMD-11943 (pre-post-powerstroke conformation of the ODAs), EMD-11944 (post-pre-powerstroke conformation of the ODAs), EMD-11945 (post-postpowerstroke conformation of the ODAs), EMD-11946 (axonemal 96-nm repeat from active

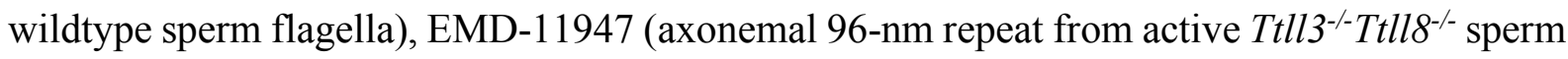
flagella), EMD-11948 (axonemal 96-nm repeat from active sperm flagella showing IDAs in pre-powerstroke conformations), EMD-11949 (axonemal 96-nm repeat from active sperm flagella showing IDAs in post-powerstroke conformations). All other data are available in the manuscript or the supplementary material. 


\section{List of Supplementary Materials}

Figures S1 - S10

Tables S1 - S3

Movies S1 - S6 


\section{Figures}

\section{Figure 1: Total absence of glycylation in $\mathrm{Ttll3}^{-/-} \mathrm{Ttll}^{-/-}$mice}

(A) Paraffin-embedded testes sections stained for tubulin acetylation (6-11B-1; green), glycylation (Gly-pep1; red), and DNA (DAPI). Nuclear staining shows normal sperm head morphology, and a normal arrangement of sperm heads of different developmental stages in the

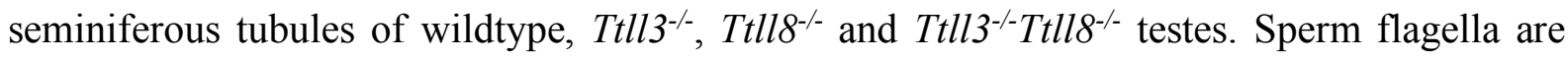
present in all genotypes (labelled by 6-11B-1), and are glycylated in wildtype and single knockout, but not in $\mathrm{Ttll}^{-/} \mathrm{Ttll}^{-/-}$sperm tails. (B) Immunoblot of sperm samples from wildtype,

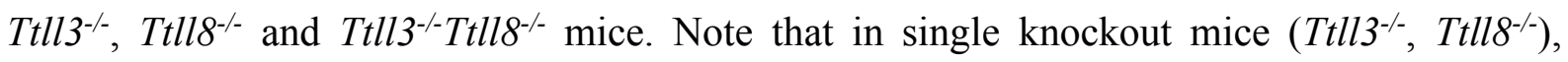
glycylation (Gly-pep1) is only partially abolished, while it is undetectable in Ttll3 $^{-/-}$Ttll $^{-/-}$ sperm. The anti- $\alpha$-tubulin antibody $12 \mathrm{G} 10$ confirmed equal tubulin load.

\section{Figure 2: Ttll3 $^{-/-}$Ttll8 $^{-/-}$mice show subfertility and reduced sperm motility}

(A) Analysis of litter size: comparison of heterozygotes $\left(\mathrm{Ttll}^{+/-} \mathrm{Ttll}^{+/-}\right)$with $\mathrm{Ttll3}^{-/-} \mathrm{Ttll}^{-/-}$mice show a reduction in the average number of pups per litter (see Fig S3E for details). (B) In vitro fertilisation assay with wildtype oocytes. The average fertilisation index of five independent experiments (Fig S4B) shows a strong decrease of $T t l l 3^{-/} T t l l 8^{-/}$sperm fertility. (C) Computerassisted sperm analyses (CASA) comparing wildtype and Ttll3 ${ }^{-/}$Ttll8 $^{-/}$sperm. Ttll3 $^{-/-}$Ttll $^{-/-}$mice show a reduced proportion of progressive sperm. In particular, Ttll $^{-/-}$Ttll $^{-/-}$sperm had reduced velocity parameters: curvilinear velocity (VCL), straight-line velocity (VSL), and average path velocity (VAP), together with reduced lateral head displacement $(\mathrm{ALH})$. The beat-cross frequency $(\mathrm{BCF})$, on the other hand, was higher in the Ttll3 $3^{--}$Ttll8 $^{-/-}$sperm. 


\section{Figure 3: Asymmetric beat of Ttll3 $^{-/-}$Ttll8 $^{-/-}$sperm flagella}

(A) Colour-coded time projections of dark-field recordings of head-tethered mouse spermatozoa in 2D: The depicted colour-coded time span (140 ms) corresponds to one beat cycle of wildtype sperm. The flagellar envelope of $T t l l 3^{-/}$Ttll $^{-/-}$sperm cells is asymmetrically displaced to the open-hook side of the head, in contrast to wildtype sperm. See also Movie S1. (B-D) Multi-parameter motility analyses of the flagellar beat using the software SpermQ. In all line graphs, solid lines indicate the time-averaged values, and dotted lines the standard deviation for different arc-length positions along the flagellum. Scatter dot plots show the time- and arclength-averaged values for individual sperm cells (dots) as well as the mean values \pm SEM (bars). (B) Mean flagellar curvature. (C) Amplitude of flagellar beat in the direction perpendicular to the head-midpiece-axis. (D) Representation of peak frequencies of the flagellar beat. Scatter plot shows the peak frequencies separately for the first $5 \mu \mathrm{m}$, and for the rest of the flagellum. 


\section{Figure 4: Altered swimming behaviour of $\mathrm{Ttll}^{-/-} \mathrm{Ttll}^{-/-}$sperm}

(A) Three-dimensional flagellar shapes used to compute the 3D swimming path of sperm cells. The shapes were extracted from experiments using tethered sperm (Fig 3A). The flagella shapes correspond to about half a beat period, but simulations were based on the complete time series. The $z$ component was assumed to be a smooth arc of constant curvature along the flagellar arc length $\left(\kappa_{\mathrm{z}}=5 \cdot 10^{-3} \mu \mathrm{m}^{-1}\right.$; projections shown in black). (B) Computed swimming paths of sperm cells using resistive-force theory. Wildtype spermatozoa are predicted to swim along a twisted ribbon, whereas $\mathrm{Ttll3}^{-/} \mathrm{Ttll}^{-/}$sperm cells are predicted to swim along a helical path. (C) Representative examples for reconstruction of 3D swimming paths from in-line holographic recordings of freely-swimming sperm. Wildtype and $T t l l 3^{-/-} \mathrm{Ttll}^{-/-}$sperm swim along a twisted ribbon and a helical path, respectively. Insets $(B, C)$ : Back view of the path in the direction indicated by the arrow. See also Fig S6 for individual sperm trajectories. (D) 3D holographic trajectory of a $\mathrm{Ttll}^{-/-} \mathrm{Ttll}^{-/-}$sperm showing the transition from a helical to a circular swimming path when reaching the upper wall of the observation chamber. See also Movie S3. (E) Colourcoded time projections of representative dark-field recordings of freely-swimming wildtype and Ttll3 $^{--}$Ttll8 $^{-/-}$sperm near the glass surface (see also Movie S2). (F) Quantification of the swimming patterns observed in (E). The graph represents the mean $( \pm \mathrm{SEM})$ of the different mice analysed. 


\section{Figure 5: The assembly of the axonemal 96-nm repeat is not affected in $\mathrm{Ttll3}^{-/-} \mathrm{Ttll}^{-/-}$sperm}

(A) 3D-isosurface rendering of the 96-nm repeats from active wildtype and ttll3 $^{-/-}$Ttll $^{-/}$sperm flagella after subtomogram averaging. All known components of the axonemal 96-nm repeat were identified in both wildtype and $T t l l 3^{-/-}$Ttll $^{-/}$, indicating that absence of glycylation did not affect their assembly. We further identified densities of structures that are not found in axonemes from other species (crimson-coloured). A barrel-shaped structure between radial spokes 1 and 2 (RS1, RS2) and a density that links the neck of radial spokes 2 and 3 (RS2, RS3). ODA: outer dynein arm; IDA: inner dynein arm; IC/LC: dynein intermediate chain / light chain; N-DRC: nexin-dynein regulatory complex. (B) Slice through subtomogram averaged wildtype and Ttll3 $^{--}$Ttll8 $8^{--}$96-nm repeats. No evident modifications to the macromolecular assembly of the axoneme were found upon depletion of tubulin glycylation. (C) Difference map

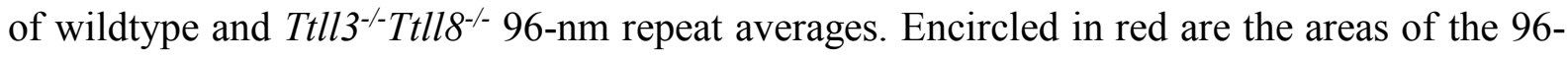
$\mathrm{nm}$ repeat that present meaningful structural differences between the two averages. These include ODAs, IDAs and part of the external wall of the B-tubule. MTBD: microtubule-binding domain 


\section{Figure 6: Distribution of ODA conformations are perturbed in $\mathrm{Ttll3}^{-/-} \mathrm{Ttll}^{-/-}$sperm flagella}

(A) Averages of all ODA subtomograms from wildtype and $T t l l 3^{-/} T_{t l l 8^{-/}}$sperm flagella (from straight axonemal segments) highlight that the structures of the dynein heads ( $\beta$-heavy chain, magenta; $\gamma$-heavy chain, green) are altered in $T t l l 3^{-/} T t l l 8^{-/}$sperm. Asterisks indicate the position of $\beta$ - and $\gamma$-heavy chains in the wildtype structure, and the corresponding coordinates in the Ttll3 $^{--}$Ttll $8^{--}$average. The Ttll $^{-/-}$Ttll $8^{--}$average shows a shift of both $\beta$ - and $\gamma$-dynein heads towards the MT plus-end (+). (B) Representative class averages of the distinct ODA conformations identified in wildtype and $T t l l 3^{-/}$Ttll $^{-/}$flagella. Isosurface rendering (top panels) and representative orthogonal and longitudinal slices (lower panels) through $\beta$ - and $\gamma$ heavy chains (colour-coded as in (A)). The numbered lines illustrate the slicing planes through the subtomogram averages in the lower panels. The superimposed schematic models show the positions of dynein $\beta$ - (magenta) and $\gamma$ - (green) AAA domains, the dynein stalks (orange lines), and the MT binding domains (orange dot). Note that pre-pre, pre-post, post-pre and post-post refer to dynein $\gamma$ - and $\beta$-heavy chain conformations, respectively (pre $=$ pre-powerstroke, post $=$ post-powerstroke). While the position of the stalk is clearly visible in the pre-pre and postpost conformations, as highlighted by the schematics, the stalks were not easily identified in the pre-post and post-pre conformations, probably because of their unstable conformation. (C) Incidence of the distinct ODA conformations in wildtype $(\mathrm{n}=2691)$ and Ttll3 $^{-/-}$Ttll $^{-/-}(\mathrm{n}=3656)$ axonemes. Ttll3 $^{-/}$Ttll $8^{-/-}$flagella show a particularly increased percentage of pre-post and postpost powerstroke conformations. (D) Distribution of the different ODAs conformations visualised in wildtype and $T t l l 3^{-/-}$Ttll $^{-/-}$axonemes shows the absence of a clear pattern of

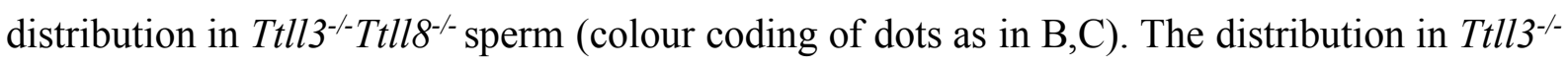

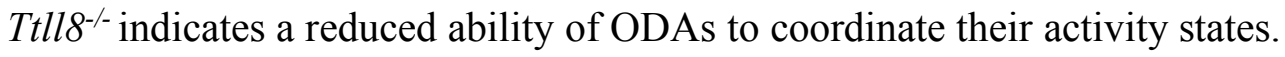



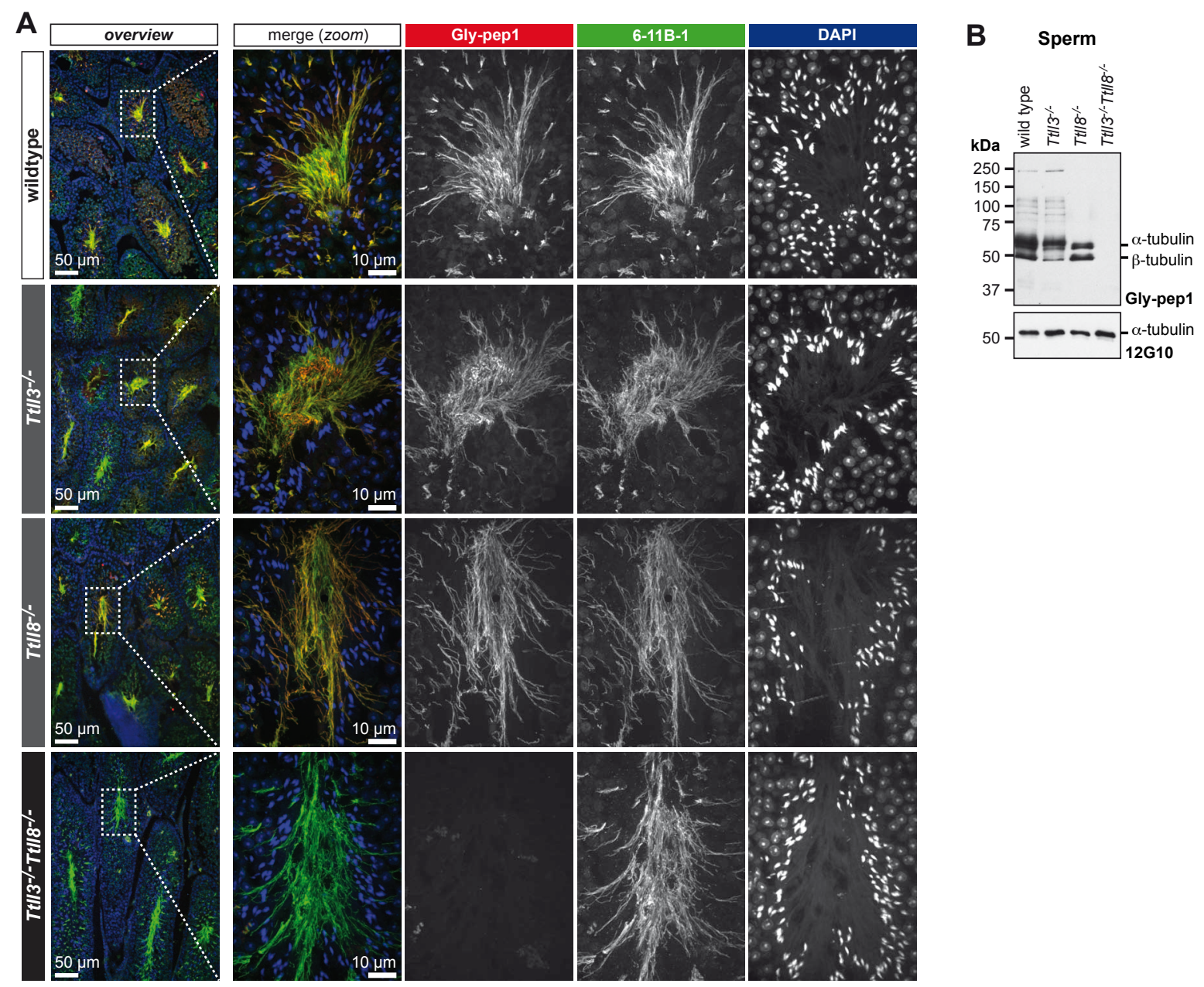


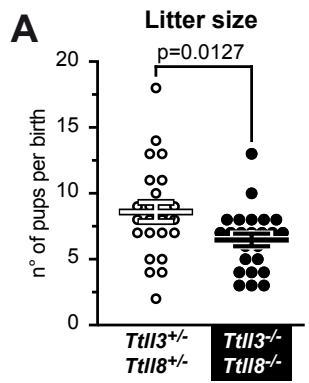

\section{B Average in-vitro fertilisation index}

C Computer-assisted sperm analyses (CASA)

progressive motility curvilinear velocity straight-line velocity
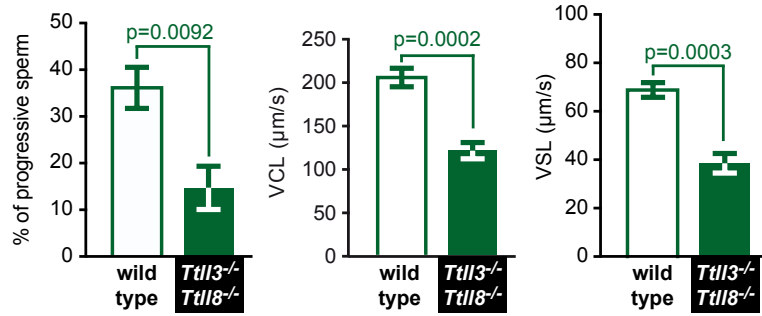

average path velocity

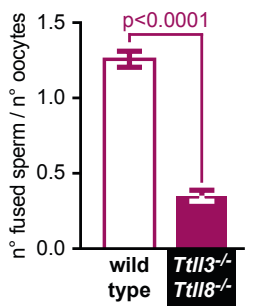

lateral head displacement

cross frequency
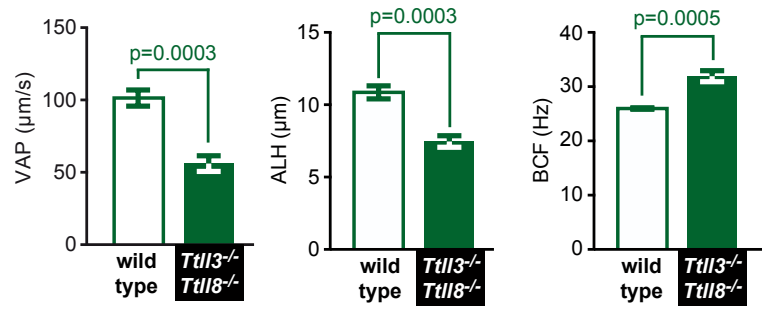

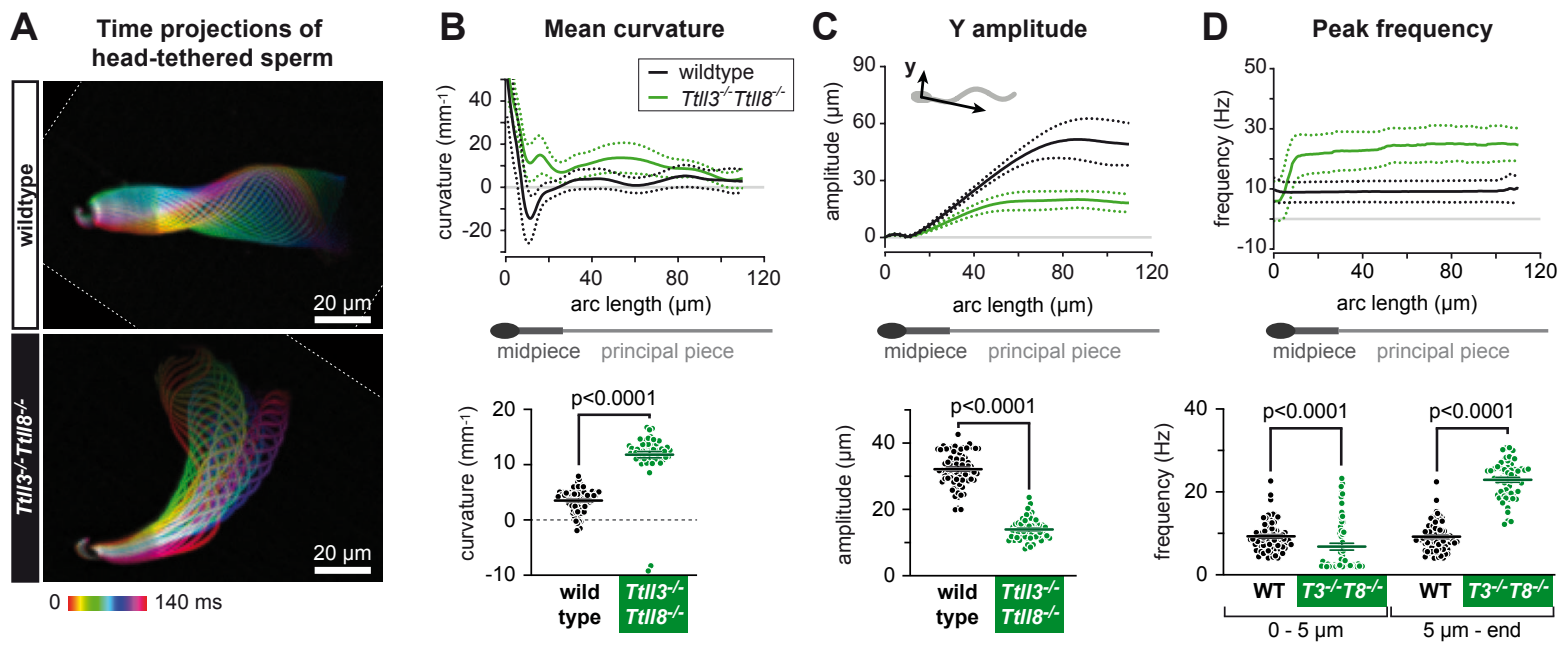

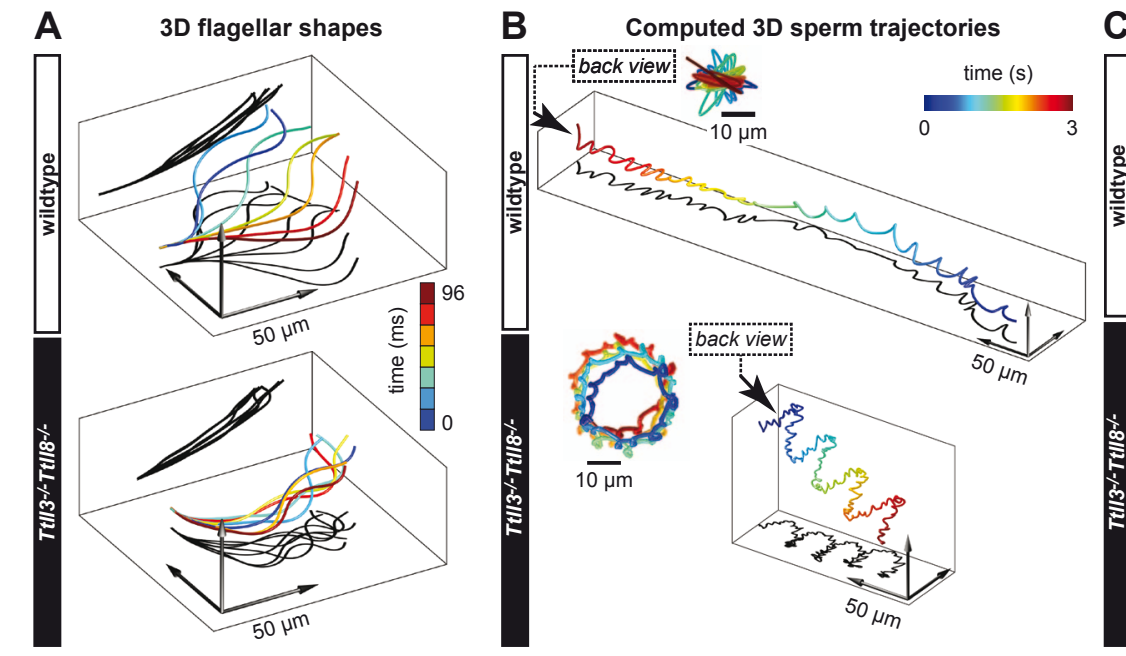

C Experimental 3D sperm trajectories
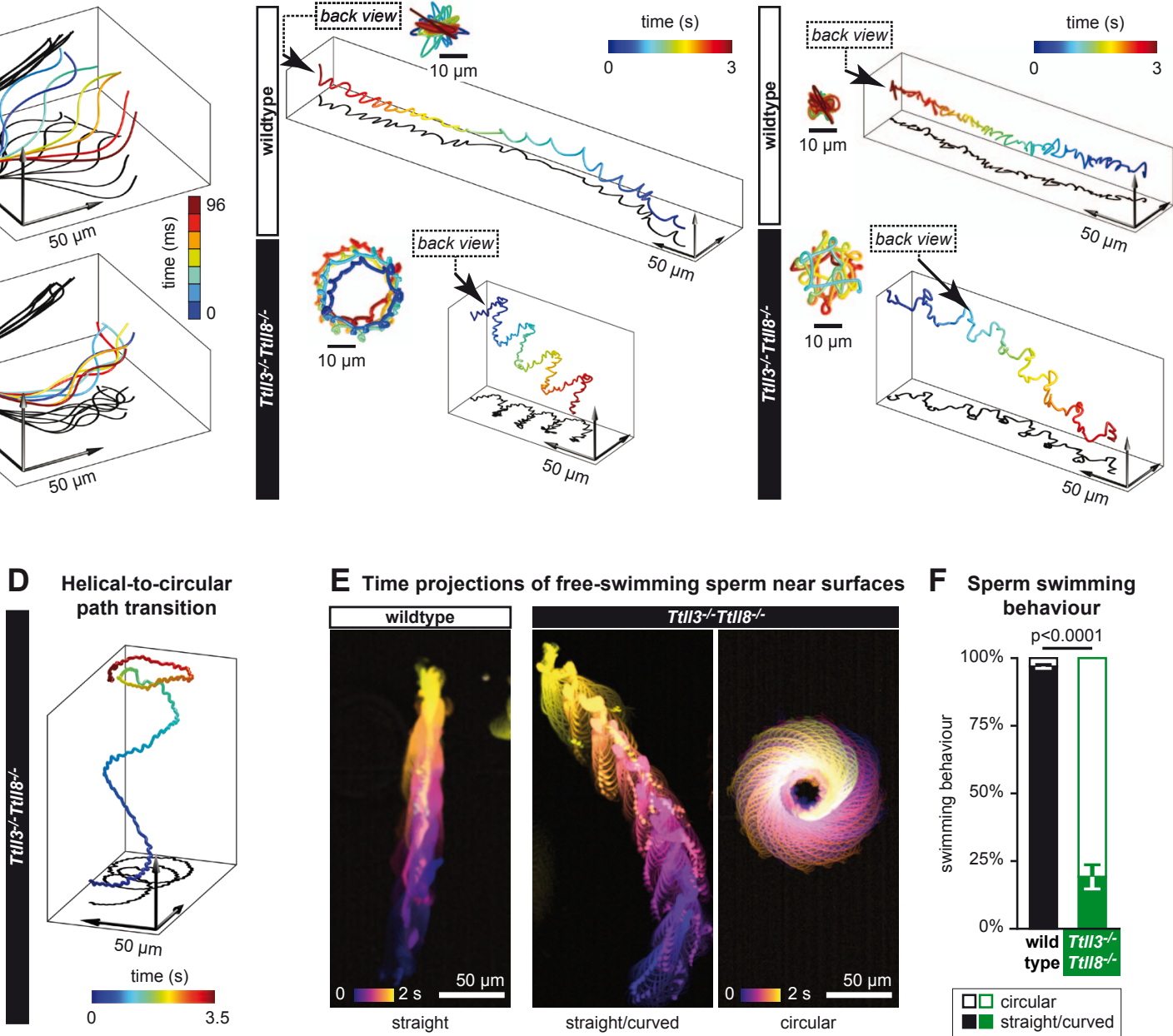


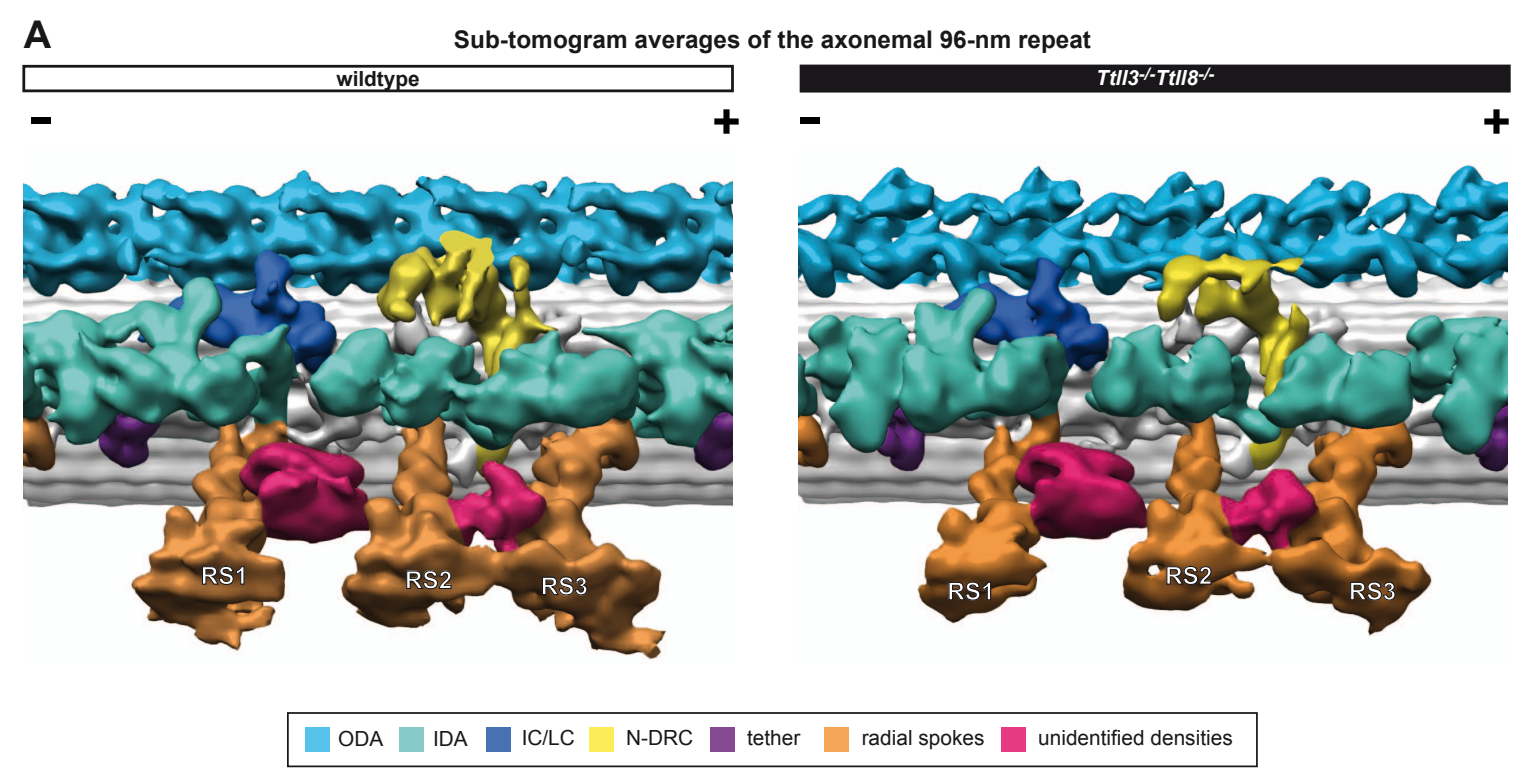

B

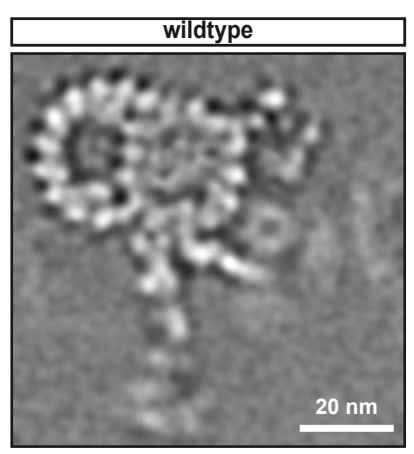

Cross-section of the 96-nm repeat

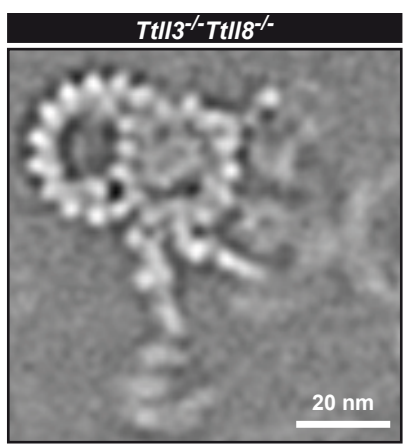

C Difference map of $96-\mathrm{nm}$ repeat averages

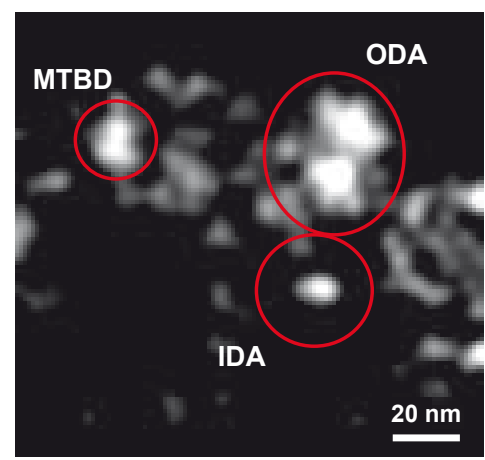



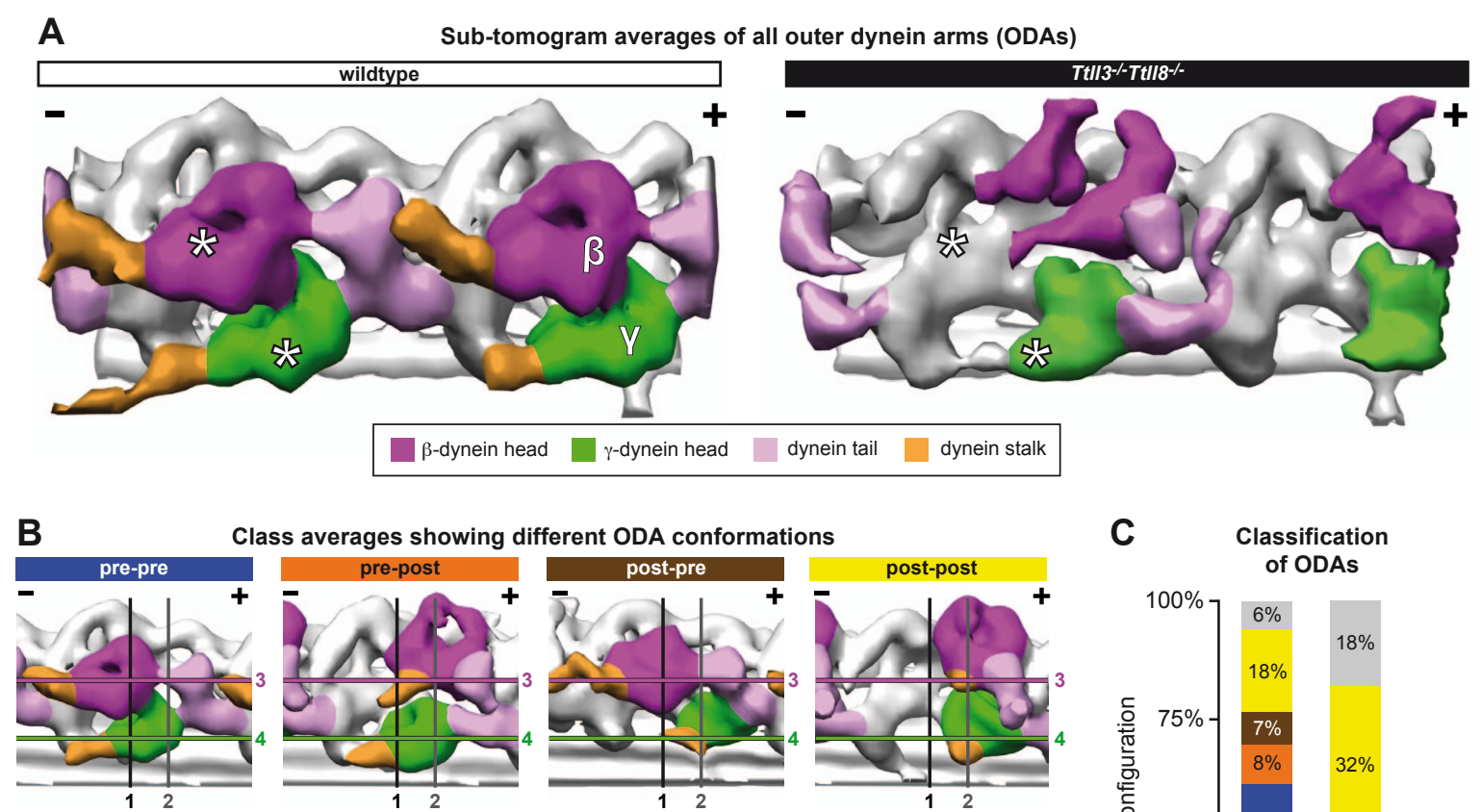

Class averages showing different ODA conformations
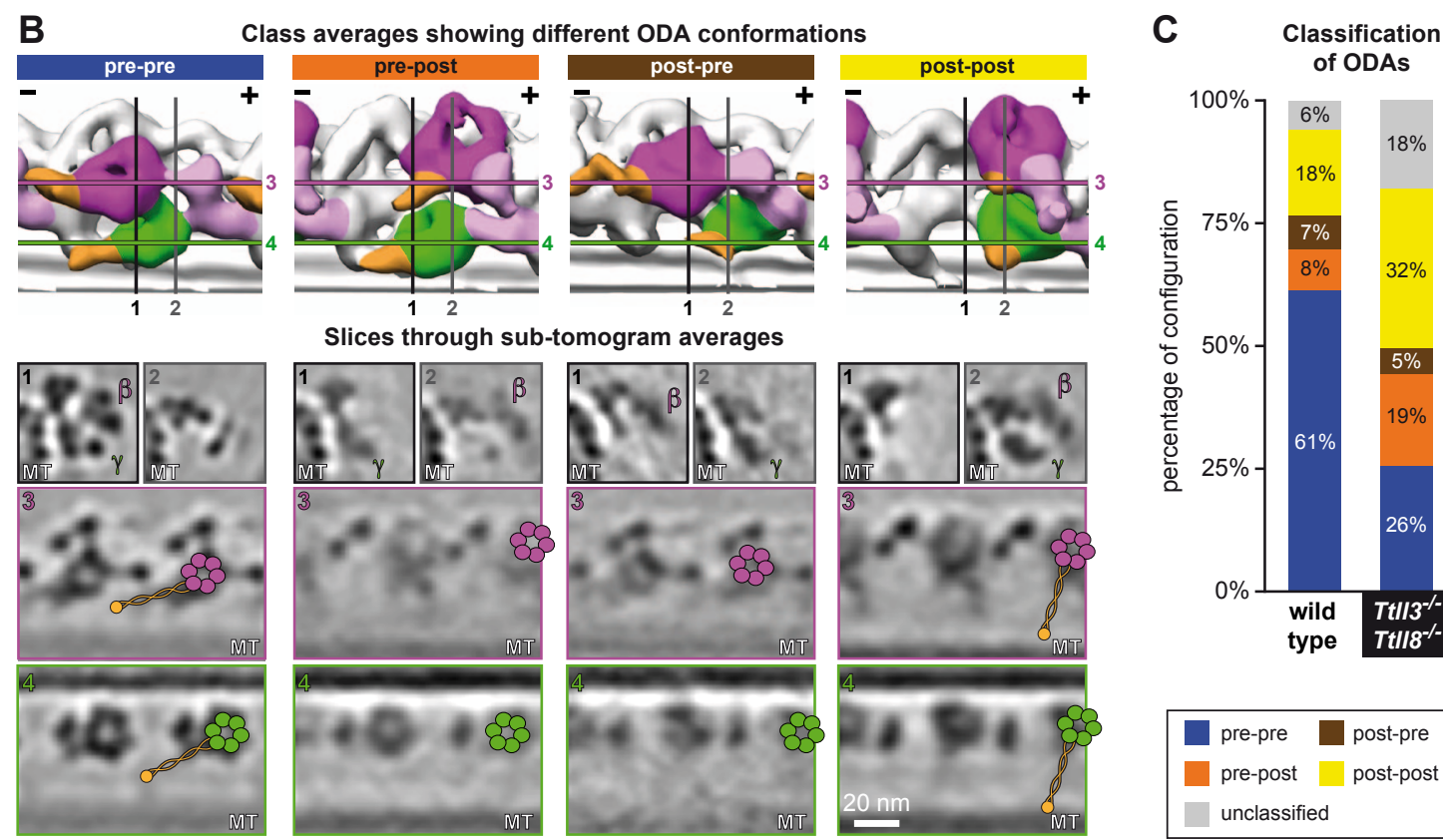

Slices through sub-tomogram averages
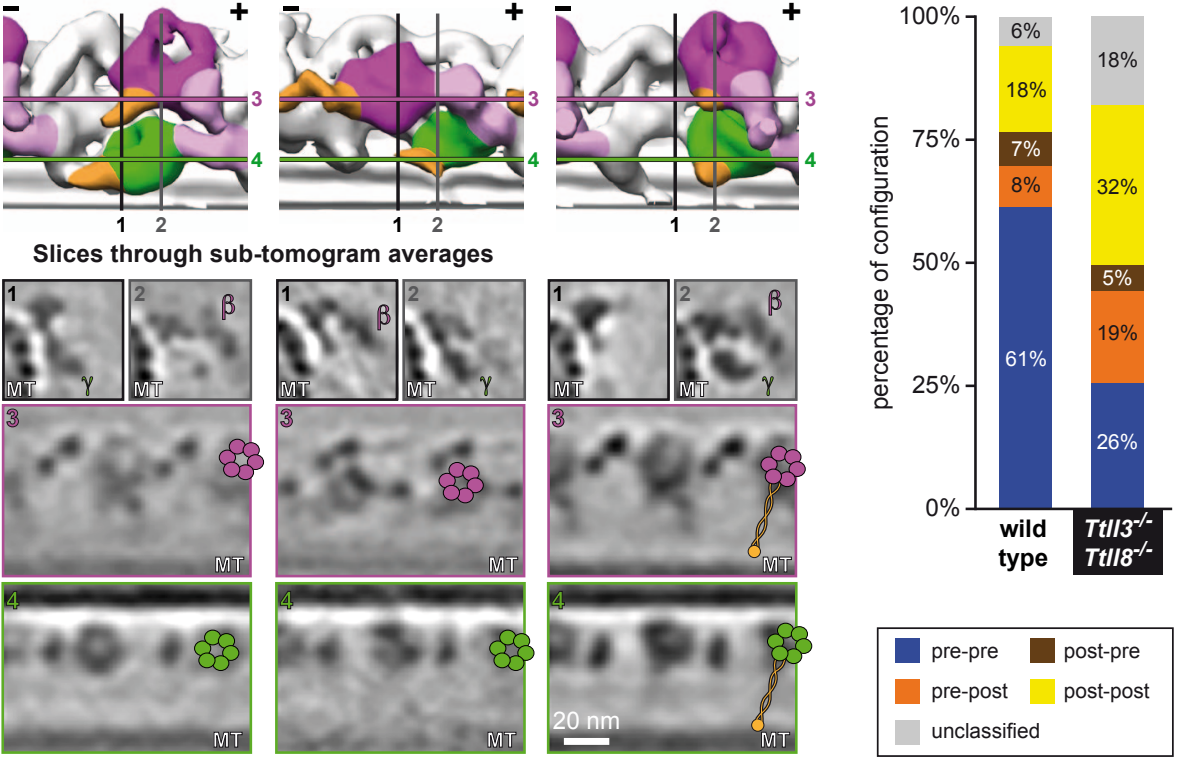

D

Distribution of ODA conformations in axonemes

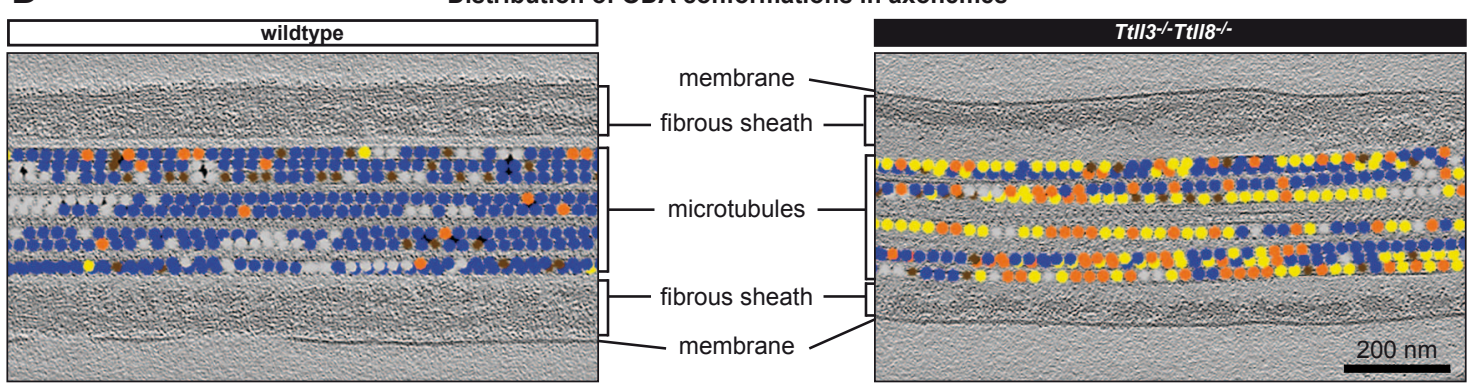




\section{Science МIAAAS}

\section{Supplementary Materials for}

Tubulin glycylation controls axonemal dynein activity, flagellar beat and male fertility

Sudarshan Gadadhar\#, Gonzalo Alvarez Viar*, Jan Niklas Hansen*, An Gong*, Aleksandr Kostarev, Côme Ialy-Radio, Sophie Leboucher, Marjorie Whitfield, Ahmed Ziyyat, Aminata Touré, Luis Alvarez*\#, Gaia Pigino*\#, Carsten Janke\#

\#correspondence to: Carsten.Janke@curie.fr, Sudarshan.Gadadhar@curie.fr, pigino@mpi-cbg.de, luis.alvarez@caesar.de

This PDF file includes:

Figures $\mathrm{S} 1-\mathrm{S} 10$

Table S2

Legends for Movies S1 - S6

Other supplementary materials for this manuscript include the following:

Movies S1 - S6

Tables S1 and S3 


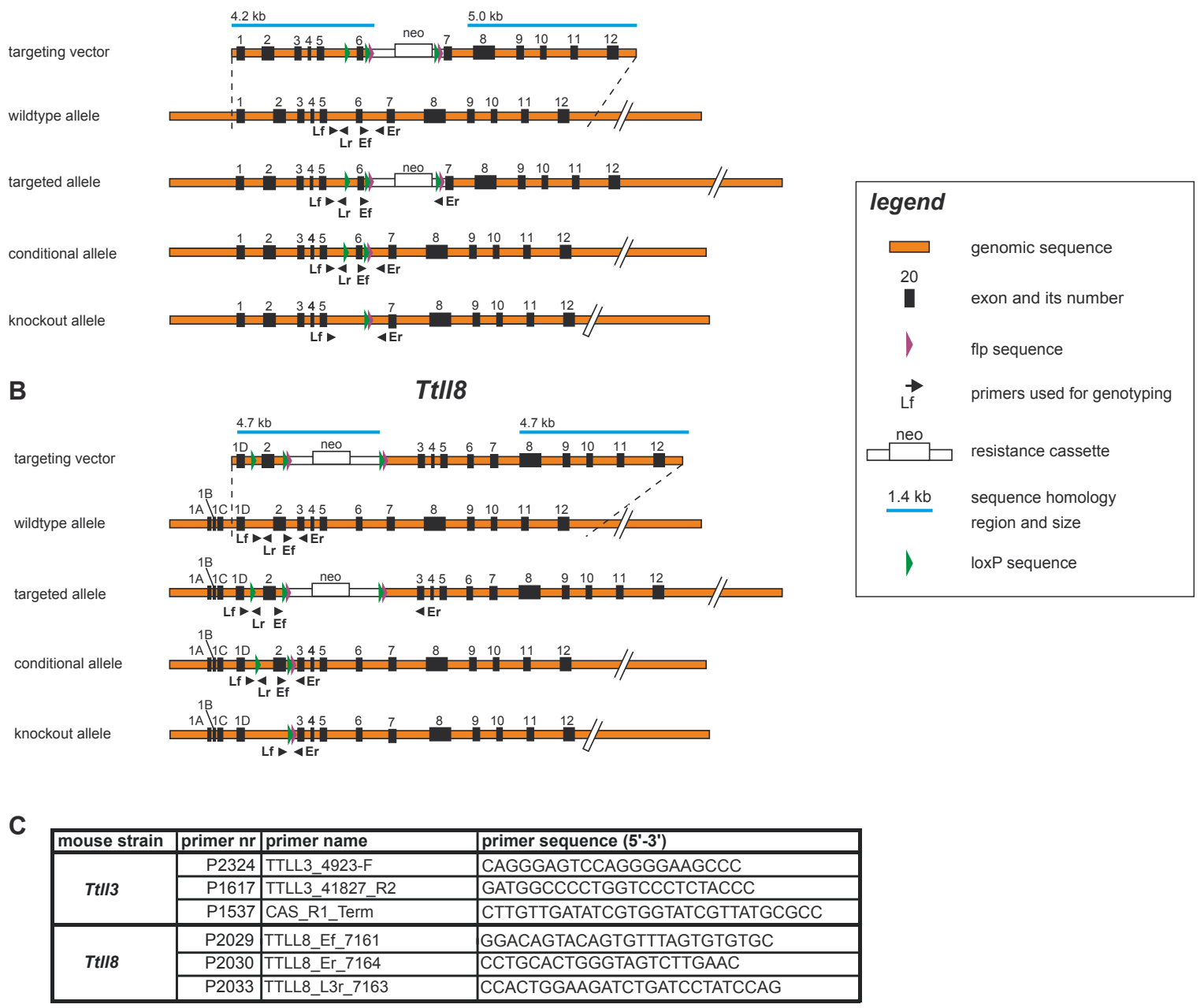

D

\begin{tabular}{|c|c|c|c|c|c|c|c|c|}
\hline \multirow{2}{*}{ mouse strain } & \multirow[t]{2}{*}{ PCR id } & \multirow{2}{*}{ PCD product size (bp) } & \multirow{2}{*}{ primer mix } & \multicolumn{2}{|c|}{ annealing } & \multicolumn{2}{|c|}{ elongation } & \multirow{2}{*}{ cycles } \\
\hline & & & & time (s) & temp $\left({ }^{\circ} \mathrm{C}\right)$ & time (s) & temp $\left({ }^{\circ} \mathrm{C}\right)$ & \\
\hline Ttll3 & \begin{tabular}{|l} 
PCR1 \\
PCR2
\end{tabular} & \begin{tabular}{|l|}
$550(\mathrm{wt})$ \\
$250(\mathrm{KO})$ \\
\end{tabular} & P2324 + P1617 & 30 & 58 & $30+1 /$ cucle & 72 & 33 \\
\hline Ttll8 & PCR1 & $241(w t)$ & P2029+P2 & & & & & \\
\hline & PCR4 & $1100(\mathrm{wt}) / 280(\mathrm{KO})$ & P2029 + P2033 & 30 & 60 & 60 & 72 & 34 \\
\hline
\end{tabular}

$\mathrm{E}$ TtII3
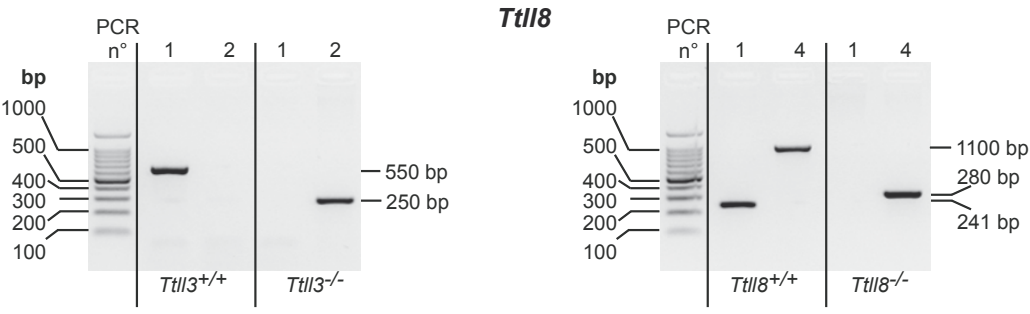

\section{Figure S1: Mouse generation and genotyping}

(A) Schematic representation (in scale) of the targeting vector used and all possible alleles of the Ttll3 gene. Orange bar: genomic DNA. Black box: exons with their corresponding number. Green and purple arrowheads: LoxP and Flp sequences, respectively. White bar: neo cassette, with the neomycin resistance gene (white box). Blue lines: zone of sequence homology for homologous recombination, with the corresponding sizes. Black arrowheads: primers used for PCR genotyping. (B) Schematic representation (in scale) of the targeting vector used and all the possible alleles of the Ttll8 gene with all the elements the same as that described above. (C) List of primers used for genotyping PCRs.

(D) PCR protocols used for genotyping. (E) Representative genotyping results of both Ttll3 and Ttll8 transgenes and their knockouts used in this study. PCR products were separated on 2\% TBE-agarose gels for $30 \mathrm{~min}$. 

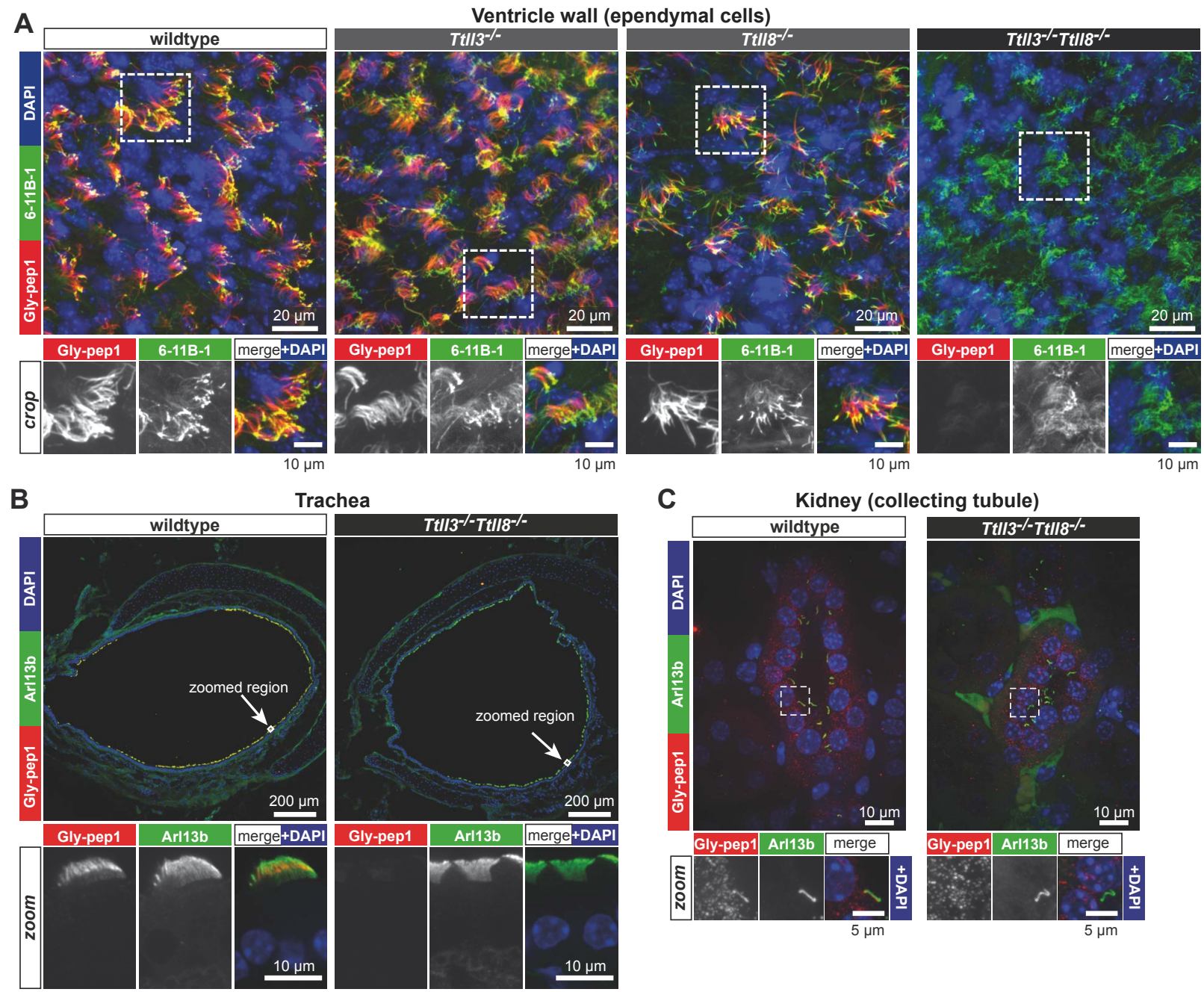

C
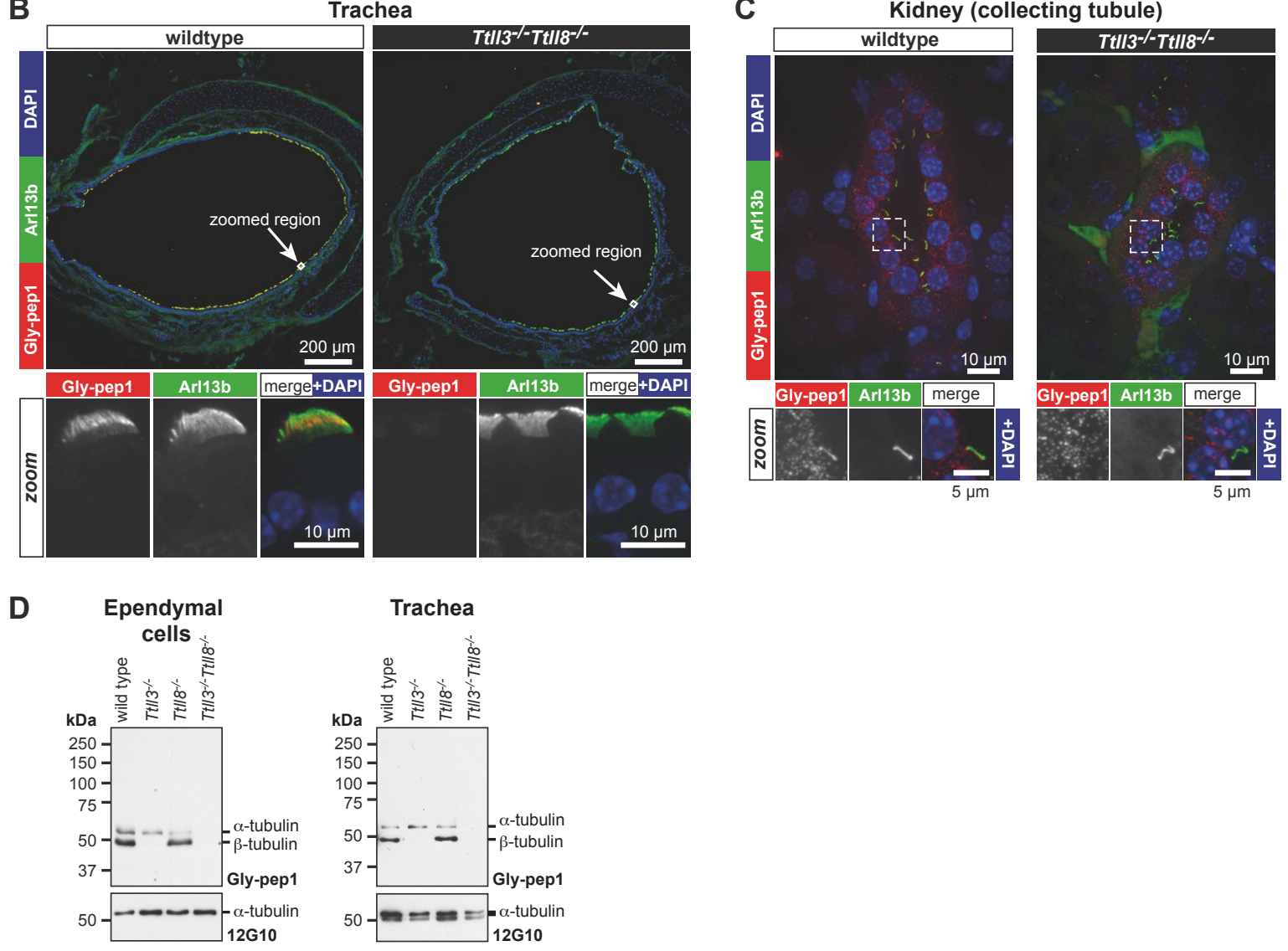

\section{Figure S2: Loss of glycylation in ciliated tissues of Ttll3 $^{--}$Ttlls $^{-/-}$mice}

(A) Immunostaining of whole-mount ventricle walls from PN15 wildtype, Ttll $3^{--}$, Ttll $8^{-/-}$, and $T t l l 3^{-/-}$Ttll $^{-/-}$ventricles stained for acetylation (6-11B-1, green), glycylation (Gly-pep1, red), and DNA (DAPI, blue). While ependymal cilia in

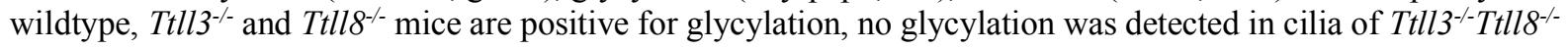
ventricles, while cilia were still present as seen with acetylated tubulin. Scale bar in the crop images: $10 \mu \mathrm{m}$.

(B) Immunostaining of paraffin-embedded sections of trachea for glycylation (Gly-pep1, red) and the cilia membrane (Arl13b; green) show the presence of epithelial cilia in both, wildtype and $T t l l 3^{--}-T_{t l l} 8^{-/}$, while glycylation is not detected in Ttll $^{-/-}$Ttll $^{-/-}$trachea. (C) Immunostaining of paraffin-embedded kidney sections for glycylation (Gly-pep1, red) and the cilia membrane (Arl13b; green) show the presence of primary cilia in both, wildtype and $T t l l 3^{-/}$Ttll $^{-/-}$kidney tubules, despite the absence of glycylation in the Ttll $^{--}$Ttll $^{-/}$cilia. (D) Immunoblots of whole-cell lysates of cultured ependymal cells, and from isolated trachea from wildtype, Ttll3 ${ }^{-/}$, Ttll $8^{-/}$and $T t l l 3^{-/}$Ttll $^{-/}$mice probed with Gly-pep1 for glycylation, and with $12 \mathrm{G} 10$ for $\alpha$-tubulin. As observed for sperm (Fig 1B), glycylation was partially lost in Ttll3 $3^{-/}$and Ttll $^{--}$, while the PTM was completely absent in $\mathrm{Ttll}^{--} \mathrm{Ttll}^{-\digamma}$ mice. 
A

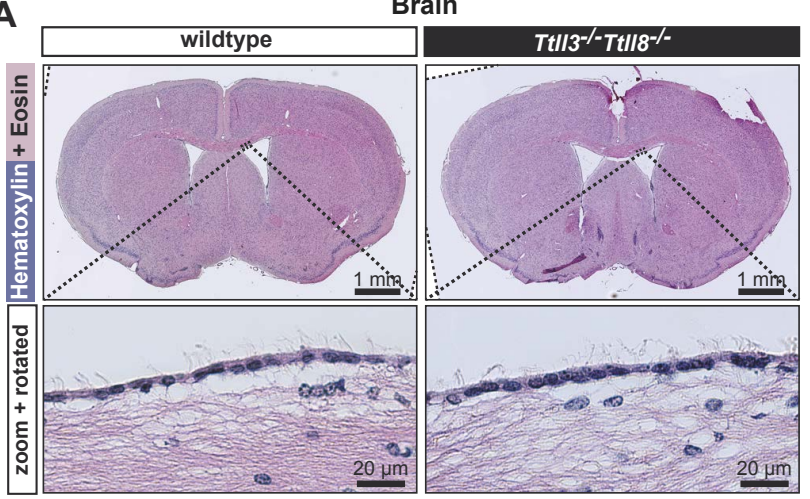

B
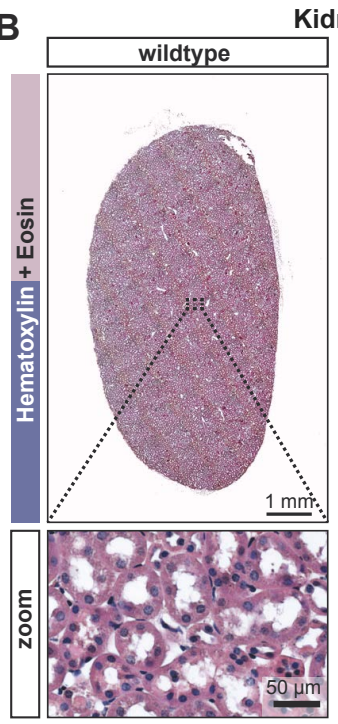

C

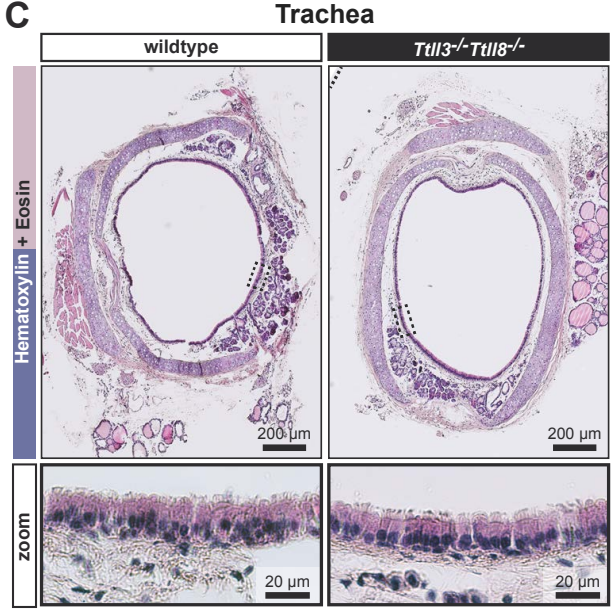

E

H
D

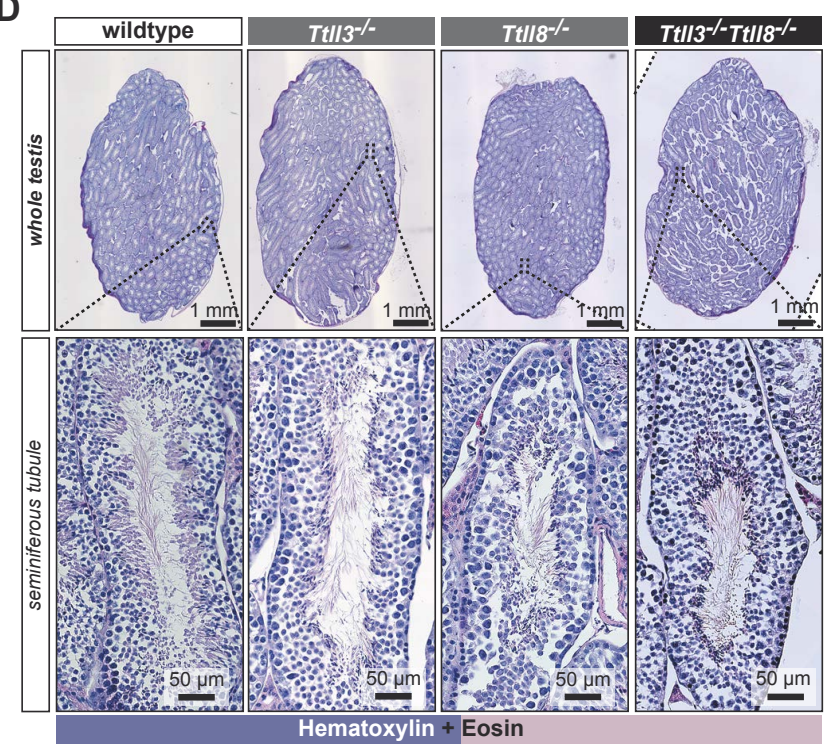

\begin{tabular}{|c|c|c|c|}
\hline \multirow{2}{*}{$0^{1 /}+$} & \multicolumn{2}{|c|}{ average litter ( \pm SD) } & \multirow{2}{*}{$\begin{array}{l}\text { litter } \\
\text { number } \\
\text { (n) }\end{array}$} \\
\hline & 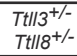 & 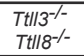 & \\
\hline 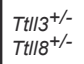 & $\begin{array}{c}8.45 \\
( \pm 3.89)\end{array}$ & 一 & 24 \\
\hline 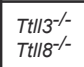 & - & $\begin{array}{c}6.46 \\
( \pm 2.36)\end{array}$ & 24 \\
\hline
\end{tabular}
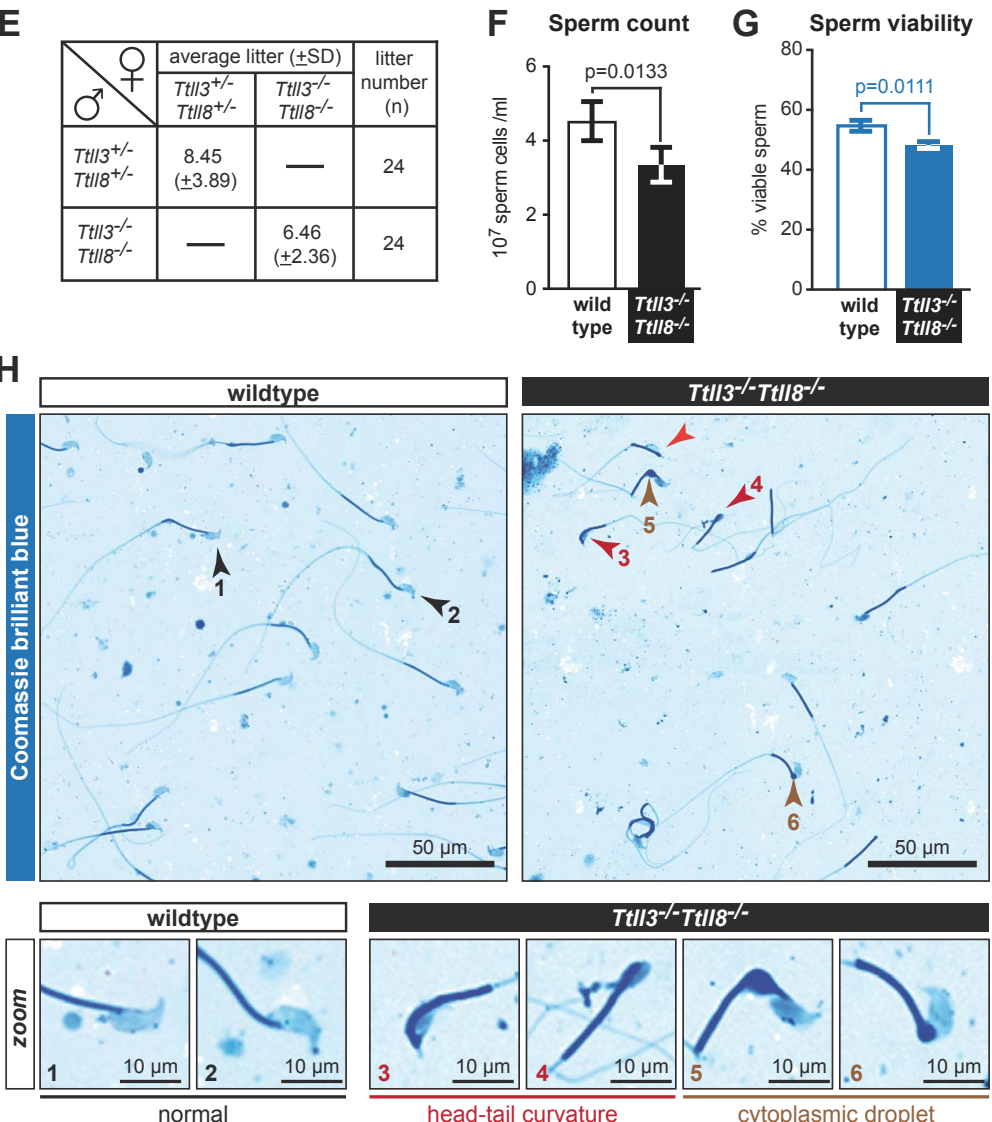

Figure S3: Absence of gross defects in Ttll $^{-/-}$Ttll $^{-/-}$mice tissues

(A-C) Hematoxylin-Eosin staining of tissues from 6-month-old wildtype and Ttll3 ${ }^{-/}$Ttll $^{-/}$mice revealed (A) no obvious defects in the tissue architecture of the brain. The ventricles of Ttll $^{-/}$Ttll $^{-/-}$mice looked normal and showed no signs of hydrocephalus. Closer observation revealed regular arrangement of multiciliated ependymal cells along the wall of the ventricle. The kidney of Ttll3 ${ }^{-/}$Ttll $8^{-/}$mice (B) showed no histological defects and absence of any cysts. The kidney tubules appear properly distributed despite the absence of glycylation. The trachea (C) appears normal in both, wildtype and $T t l l 3^{-/}$Ttll $^{-/-}$mice with proper arrangement of the columnar multiciliated epithelia. (D) Hematoxylin-Eosin stained testes from 6-month-old wildtype, Ttll $3^{--}$, Ttll $^{-/-}$and $T t l l 3^{-/-}$Ttll $^{-/-}$mice reveal no obvious defects in the tissue architecture of testes. Seminiferous tubules were of the same size, and the arrangement of developing sperm cells within 
the tubules appeared normal, indicating no defects in the process of spermatogenesis. (E) Numerical values of the litter

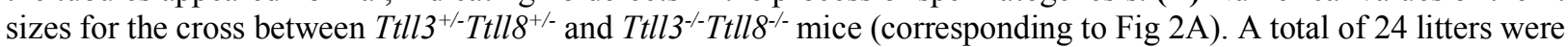
quantified for each mating scheme. (F) Counting of mature spermatozoa obtained from the cauda epididymides of wildtype and $\mathrm{Ttll}^{-/-} \mathrm{Ttll}^{-/-}$mice revealed no significant alteration in the total sperm count of $T t l l 3^{-/} \mathrm{Ttll}^{-/-}$mice. (G)

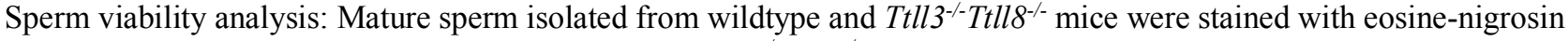
and counted. Sperm viability was slightly reduced in $T t l l 3^{--} T_{t} l l 8^{-/}$mice. (H) Smears of mature spermatozoa from wildtype and $\mathrm{Ttll}^{-/-} \mathrm{Ttll}^{-/-}$mice were stained with Coomassie brilliant blue. Note that $T t l l 3^{-/} \mathrm{Ttll}^{-/}$spermatozoa show morphological defects such as abnormal curving of the flagellum around the sperm head (red arrowheads, zoom panels 3 , 4) and the retention of cytoplasmic droplets (brown arrowheads, zoom panels 5, 6).

A

Schematic representation of CASA measuremets

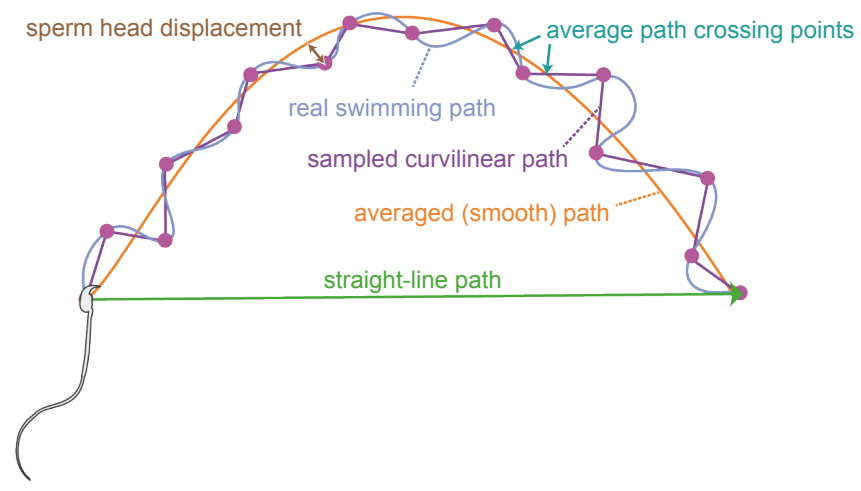

Output parameters

Real swimming velocity

Curvilinear velocity (VCL; $\mu \mathrm{m} / \mathrm{s}$ )

Averaged path velocity (VAP; $\mu \mathrm{m} / \mathrm{s}$ )

Straight-line velocity (VSL; $\mu \mathrm{m} / \mathrm{s}$ )

Mean straightness (STR; VSL/NAP; \%)

Mean linearity (LIN; VSL/VCL; \%)

Mean amplitude of lateral head displacement (ALH; $\mu \mathrm{m})$

Mean beat cross frequency $(\mathrm{BCF} ; \mathrm{Hz})$
B

Individual experiments in-vitro fertilisation index

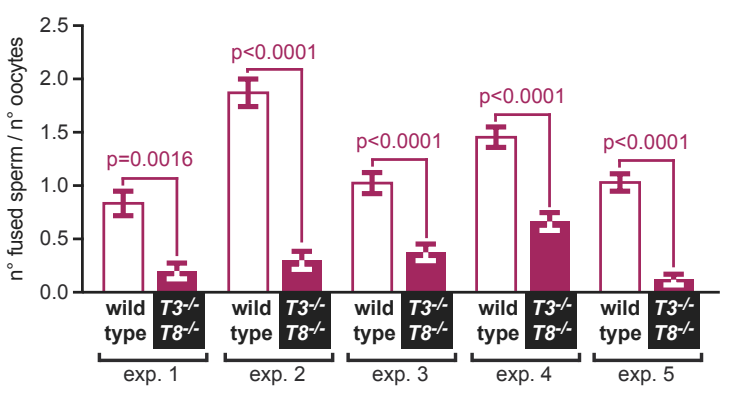

C

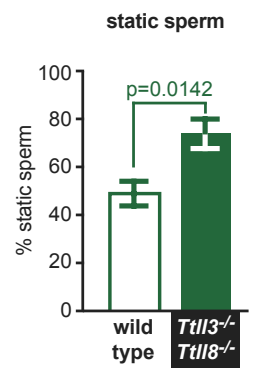

mean linearity

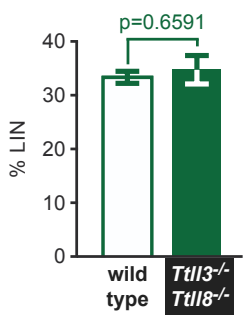

mean straightness

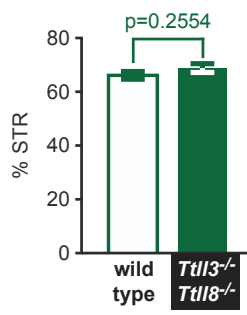

Figure S4: Ttll3 $^{-/-}$Ttlls $^{-/-}$mice are subfertile with sperm having reduced progressive motility

(A) Schematic representation of sperm motility parameters analysed by computer-assisted sperm analyses (CASA). The real swimming path (blue) of a single sperm cell is sampled at the acquisition frequency of the recording camera as the curvilinear path (pink). This swimming path is further averaged into a smooth curve (orange) and superimposed onto the wiggling path due to the periodic beating of the cell. The net forward motion of the sperm cell after a specific time interval (typically $1 \mathrm{~s}$ in CASA) is the straight-line path (green). The velocities corresponding to these different paths are depicted in the scheme, which include curvilinear velocity (VCL), straight-line velocity (VSL) and average path velocity (VAP). The ratios of these velocities are used to quantify the path linearity (LIN; VSL/VCL) and straightness of the average path (STR; VSL/VAP). CASA also analyses the displacement of the sperm head, determined as the amplitude of lateral head displacement (ALH), and the time-avergaed frequency at which the curvilinear path crosses the average path: the beat-cross frequency (BCF). (B) In vitro fertilisation test: Individual experiments of the in vitro fertilisation analysis performed with wildtype and ttll $^{-/}$Ttll $^{-/-}$mice. An average of all the experiments depicted here is shown in Fig 2B.

(C) Analysis of the motility of wildtype and $T t l l 3^{--}$Ttll $^{-/-}$sperm by CASA: Various parameters of sperm motility were

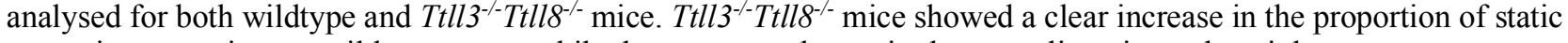
sperm in comparison to wildtype sperm while there was no change in the mean linearity and straightness. 

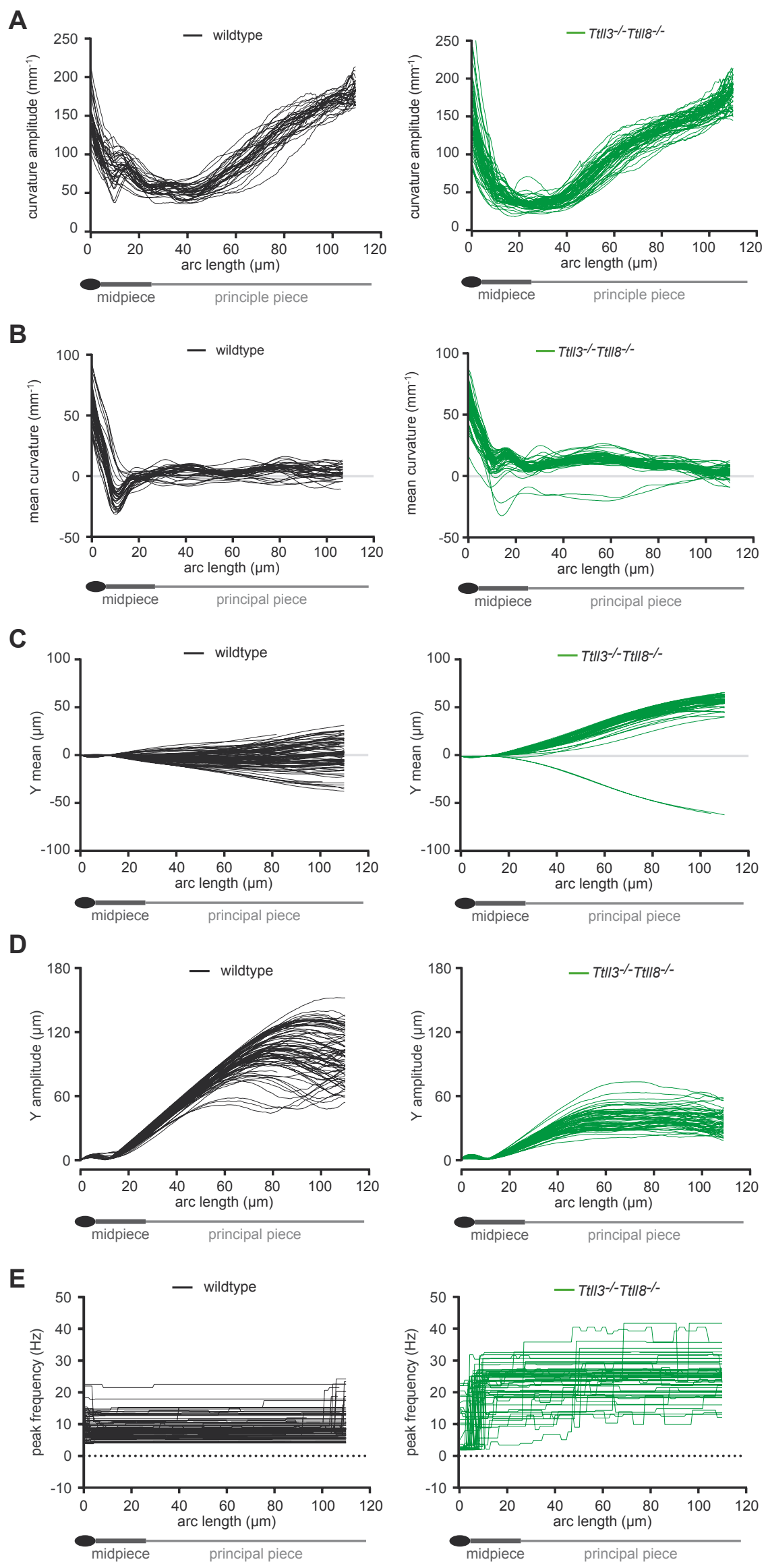
\&Figure S5: Extended data for 2D analyses of the flagellar beat

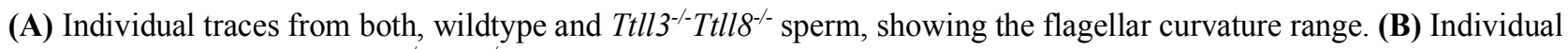
traces from wildtype and Ttll $^{-/}{ }^{-}$Ttll $8^{-/}$sperm used to calculate the mean $( \pm \mathrm{SD})$ for the analyses of mean flagellar curvature (Fig 3B). (C) Individual traces from wildtype and Ttll3 ${ }^{-/}$Ttll $8^{-/}$sperm for the time-averaged flagellar position in the direction perpendicular to the head-midpiece-axis (Y average). (D) Individual traces from wildtype and $T_{t l l 3^{-}-T t l l 8^{-}}$ ${ }^{\prime}$ sperm used to calculate the mean $( \pm \mathrm{SD})$ for the analyses of the flagellar amplitude in the direction perpendicular to the head-midpiece-axis (Y amplitude; Fig 3C). (E) Individual traces from wildtype and Ttll $3^{-/}$Ttll $^{-/-}$sperm used to calculate the mean $( \pm \mathrm{SD})$ for flagellar peak frequency (Fig 3D).
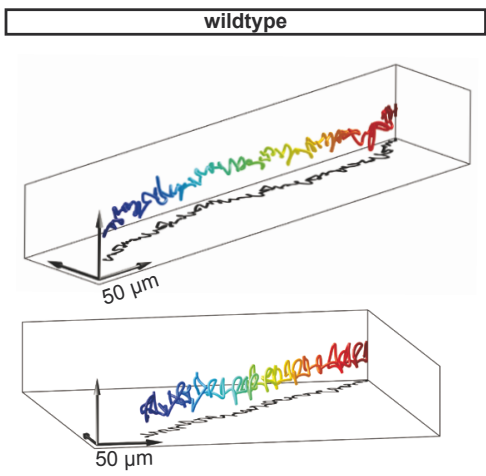

Ttll1/-Tt/18
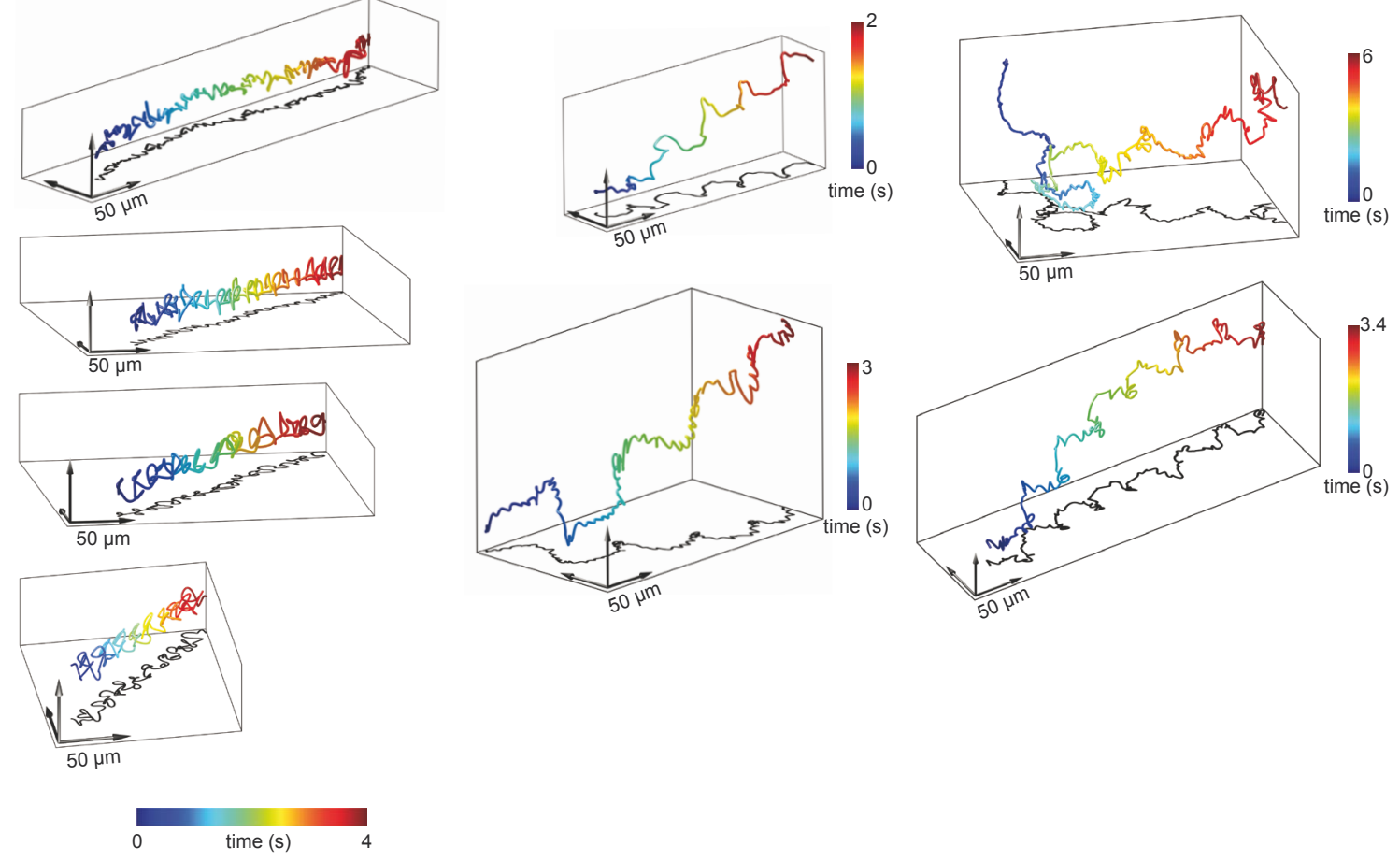

Figure S6: Extended data for 3D motility analyses

Gallery of 3D swimming paths of individual wildtype and $T t l l 3^{--}$Ttll $^{-/}$sperm recorded using high-speed in-line holography. Time is colour-coded. 


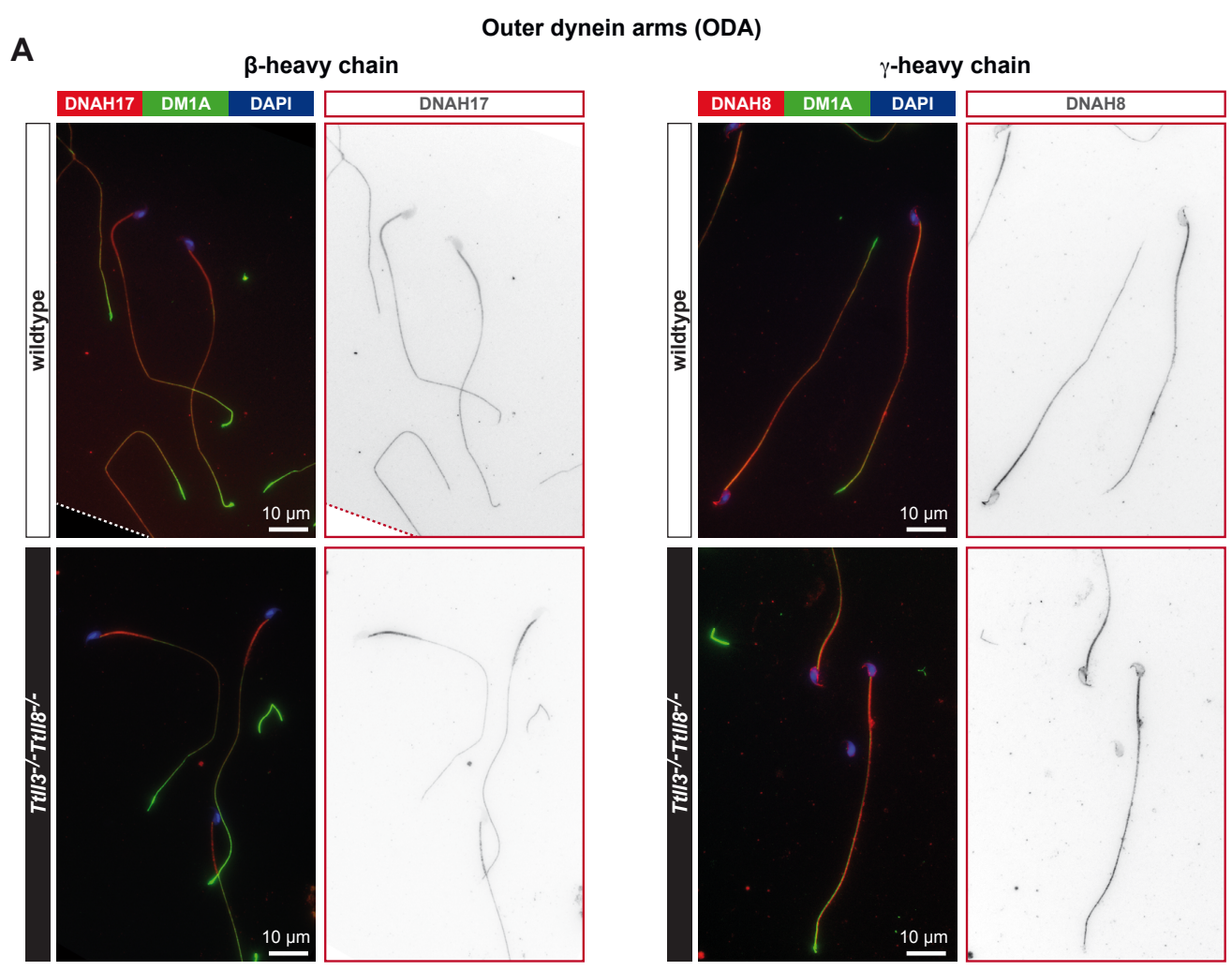

B

IDA-fB-heavy chain

Inner dynein arms (IDA)
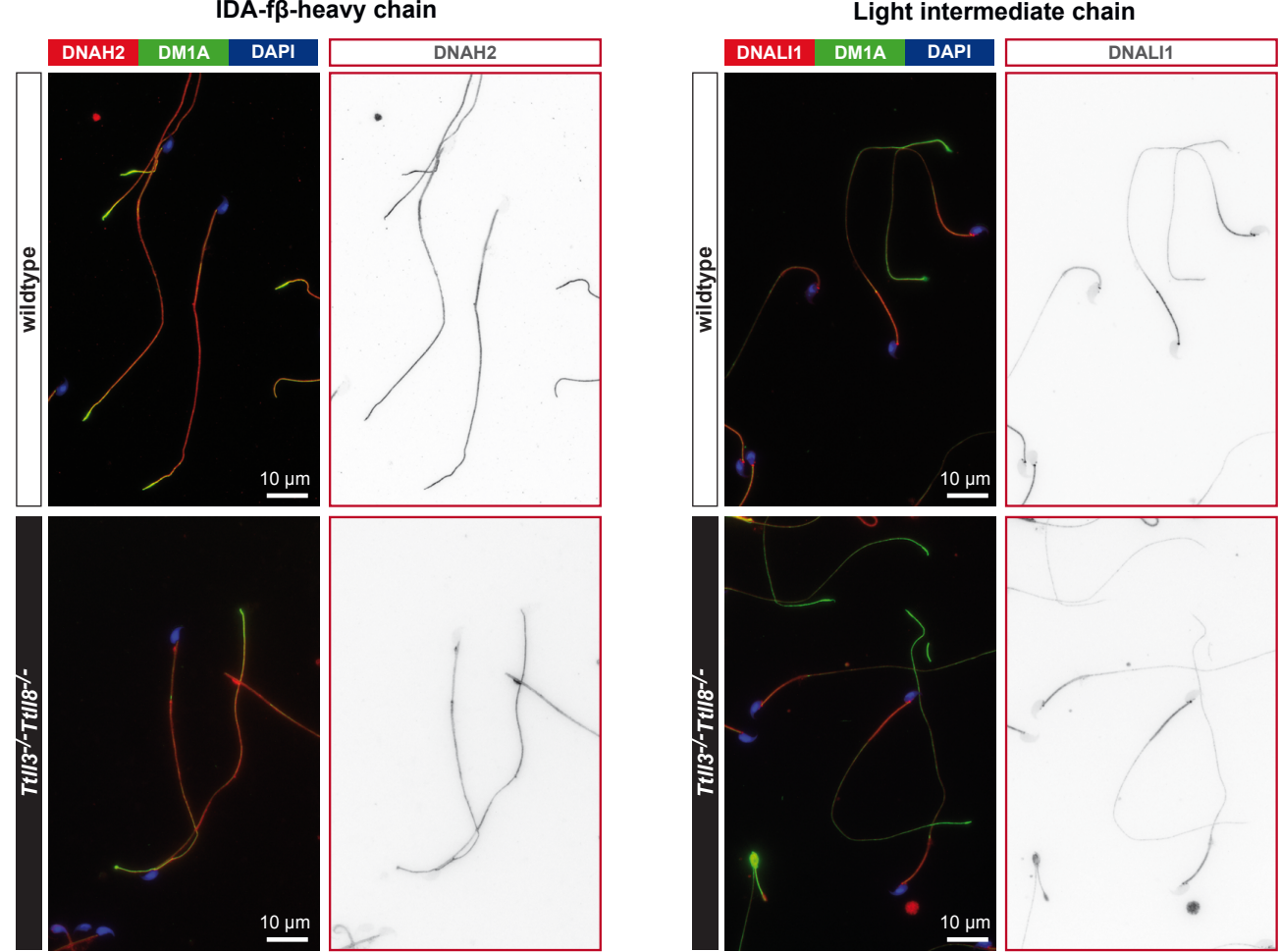

Figure S7: Distribution of axonemal dynein isoforms along flagella is not affected in Ttll3 $^{-/}$Ttlls $^{-/}$sperm

Immunostaining of wildtype and Ttll3 ${ }^{-/}$Ttll $^{-/}$sperm smears for (A) ODAs: DNAH17 ( $\beta$-heavy chain; red) and DNAH8 ( $\gamma$-heavy chain; red), and (B) IDAs: DNAH2 (IDA-f $\beta$; red) and DNALI1 (light intermediate chain; red). Microtubules are visualised by $\alpha$-tubulin staining (DM1A; green), and DNA with DAPI (blue). Nuclear staining shows normal sperm head morphology. The negative greyscale images for DNAH8 and DNAH17 (A), as well as for DNAH2 and DNALI1 and show that the distribution of ODAs and IDAs is identical in flagella of wildtype and Ttll3 ${ }^{-/}$Ttll $^{-/-}$sperm. 


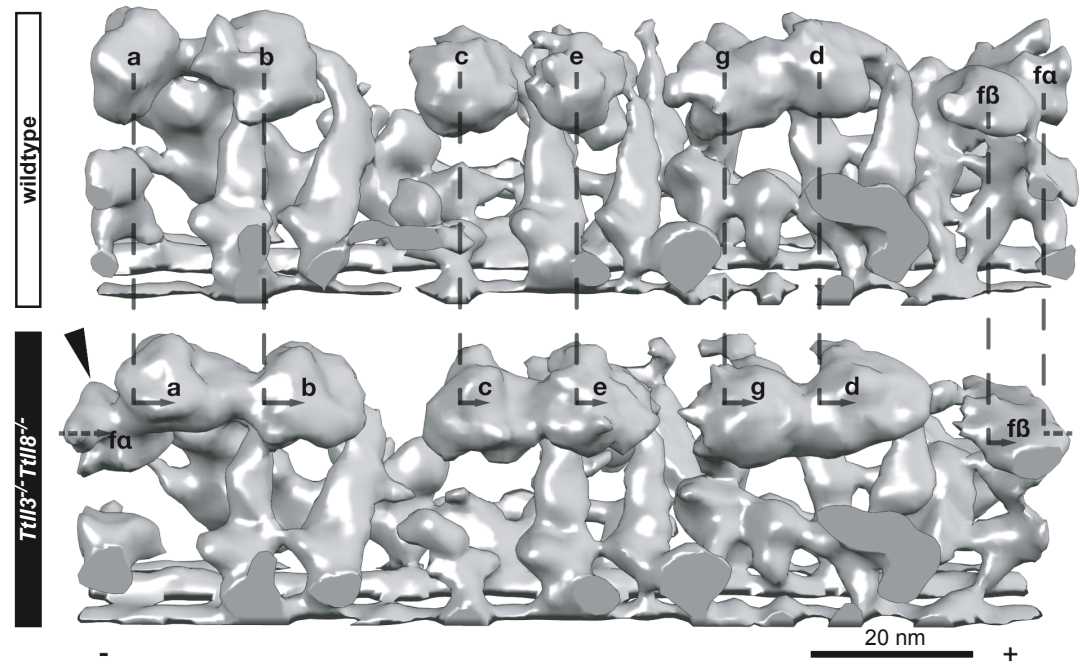

B

Class averages of inner dynein arms (IDAs)

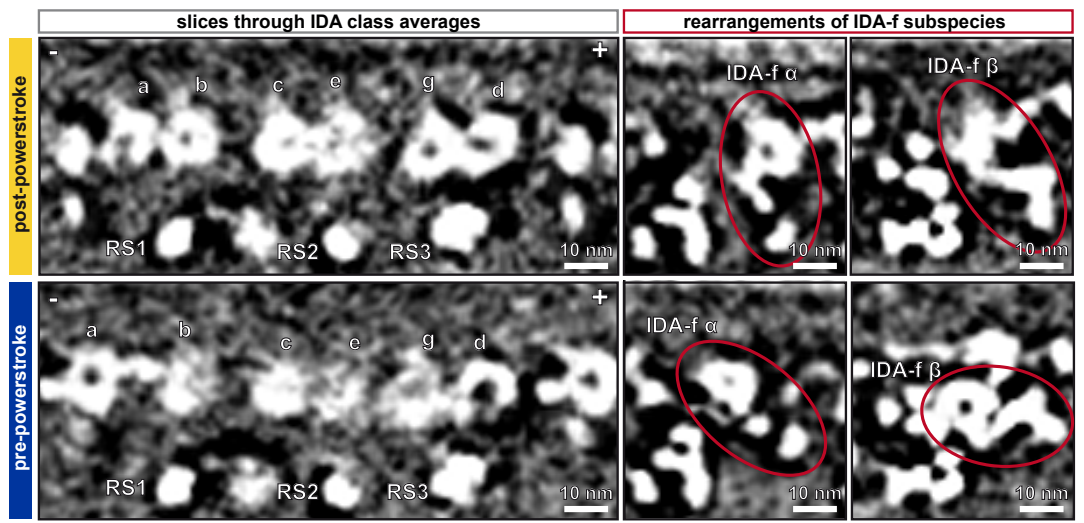

C Classification of IDAs

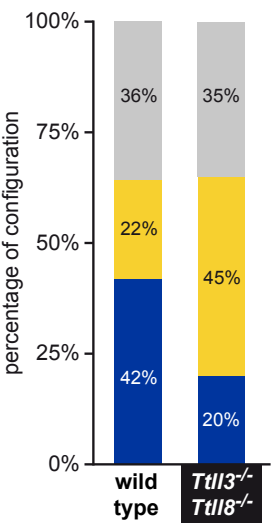

pre-powerstroke post-powerstroke unclassified

D

Distribution of IDA conformations in axonemes

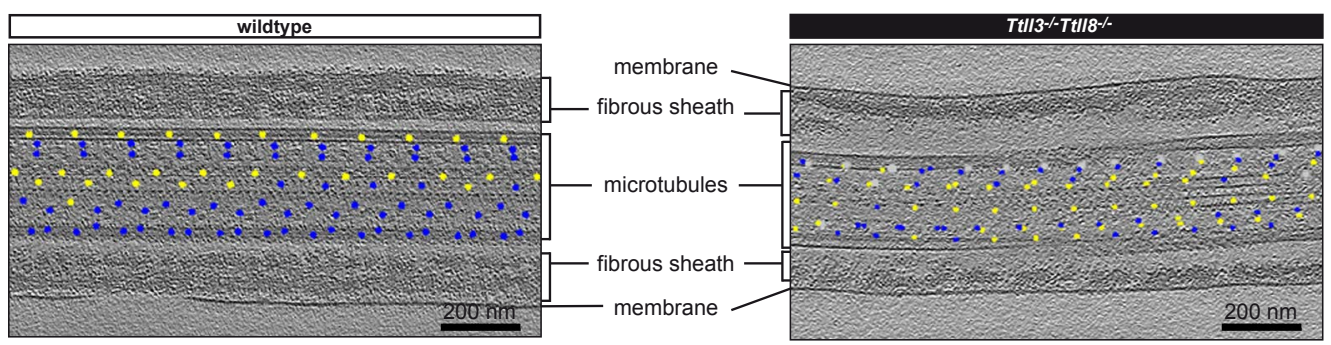

Figure S8: Inner dynein arms (IDAs) are affected in $\mathrm{Ttll3}^{-/-} \mathrm{Ttll}^{-/-}$sperm

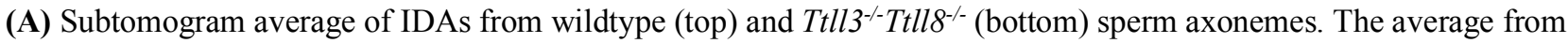
the $T t l l 3^{-/-}$Ttll $^{-/-}$sperm indicate that all IDA heavy chains are shifted towards the MT plus-end (+) as compared to wildtype. For clarity, the different IDAs are only marked by their subtype-classifying letter (a: IDA-a etc.). The largest shift is observed for IDA-f $\alpha$. Similar shifts are known from the post-powerstroke conformation of IDAs in sea urchin sperm (34). Dashed lines: approximate position of the AAA domain centre of IDAs in wildtype axonemes; arrows: shift of the AAA domains between wildtype and $T t l l 3^{-/-} T_{t l l 8^{-/}}$axonemes. (B) Slices through class averages generated from IDA classification showing their overall transition between pre-powerstroke and post-powerstroke conformations (left). Particularly consistent rearrangements of IDA-f subspecies (encircled in red) between the two classes are shown in the 
right panels. (C) Incidence of the distinct IDA conformations in wildtype ( $\mathrm{n}=960)$ and $\mathrm{Ttll}^{-/-} \mathrm{Ttll} \mathrm{S}^{-/-}(\mathrm{n}=600)$ axonemes. Unclassifiable subtomograms were excluded. $T t l l 3^{-/-}$Ttll $^{-/-}$flagella have particularly increased percentage of postpowerstroke conformations. (D) Distribution of the different IDA conformations visualised in wildtype and Ttll $3^{-/-}$Ttll $^{-/-}$ axonemes shows clear reduction of pre-powerstroke conformations, coupled to increased post-powerstroke conformations in $\mathrm{Ttll}^{-/-} \mathrm{Ttll}^{-/-}$sperm (colour coding of dots as in C).
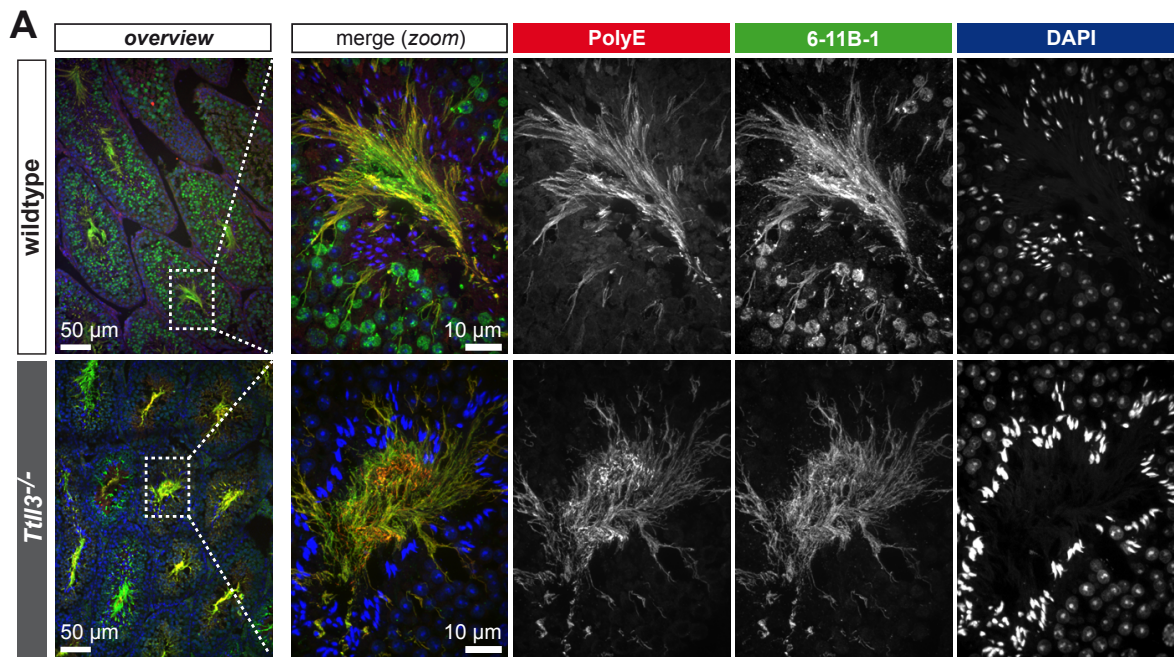

B
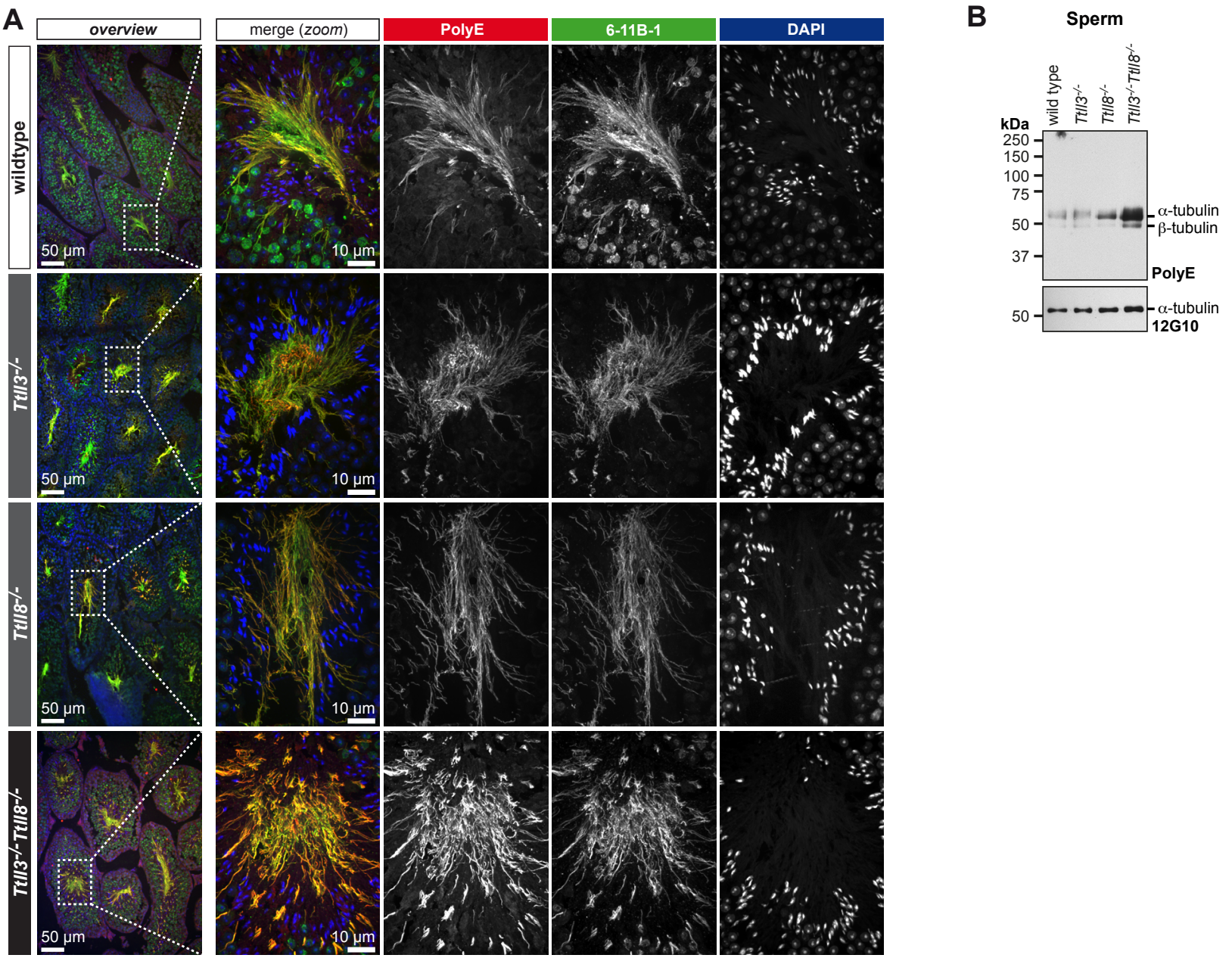

Figure S9: Loss of glycylation leads to increased glutamylation in $\mathrm{Ttll3}^{-/-} \mathrm{Ttll}^{-/-}$sperm

(A) Paraffin-embedded testes sections stained for tubulin acetylation (6-11B-1; green), glutamylation (PolyE; red), and DNA (DAPI). Nuclear staining shows normal sperm head morphology and a normal arrangement of sperm heads of different developmental stages in the seminiferous tubules of wildtype, Ttll3 ${ }^{-/}$, Ttll $^{-/-}$and Ttll $3^{-/-}$Ttll $^{-/-}$testes. Flagella from Ttll $^{-/-}$Ttll $^{-/-}$sperm show increased polyglutamylation compared to the wildtype and the single knockout sperm.

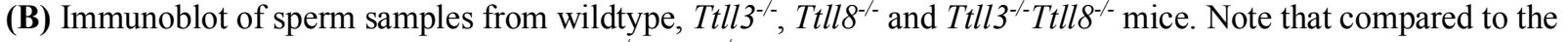
wildtype and the single knockout mice $\left(\right.$ Ttll $^{-/-}$, Ttll $\left.^{-/-}\right)$, there is a substantial increase in polyglutamylation (PolyE) of $\alpha-$ tubulin and a mild increase in $\beta$-tubulin in the $T t l l 3^{-/-}$Ttll $^{-/-}$sperm. The anti- $\alpha$-tubulin antibody $12 \mathrm{G} 10$ confirmed equal tubulin load. 

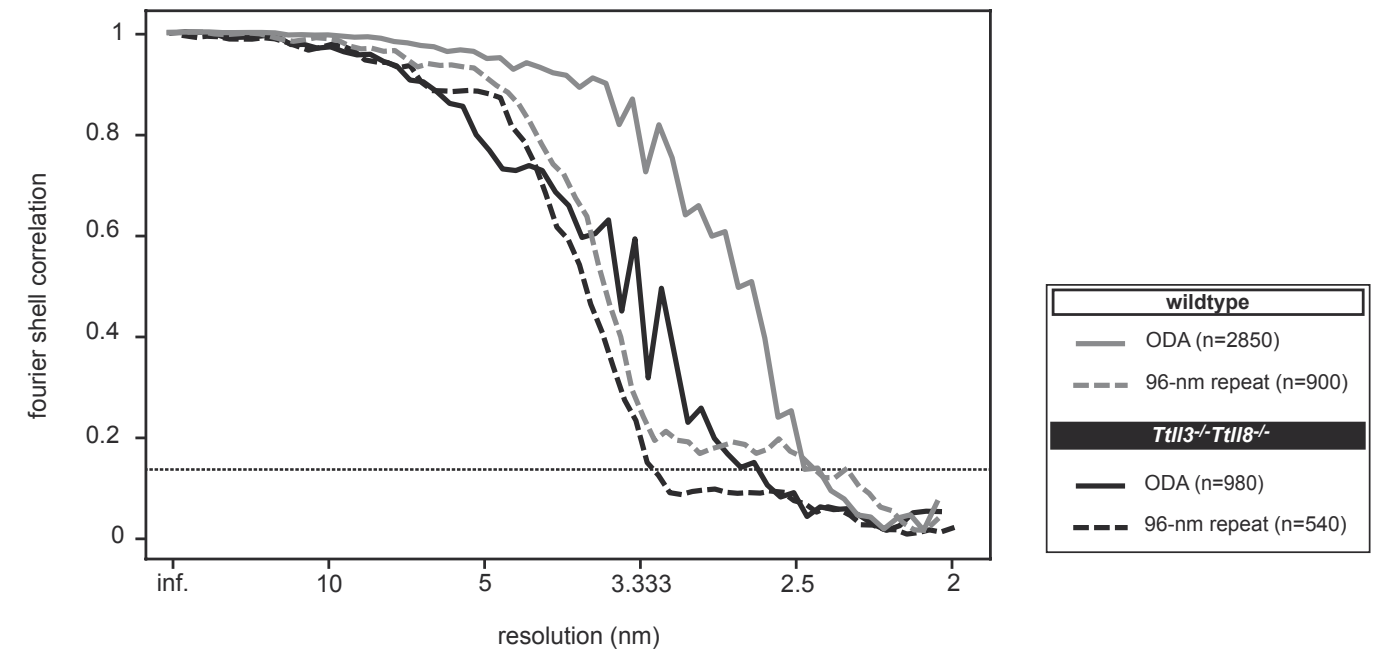

B Fourier shell correlation curves for different ODA conformations

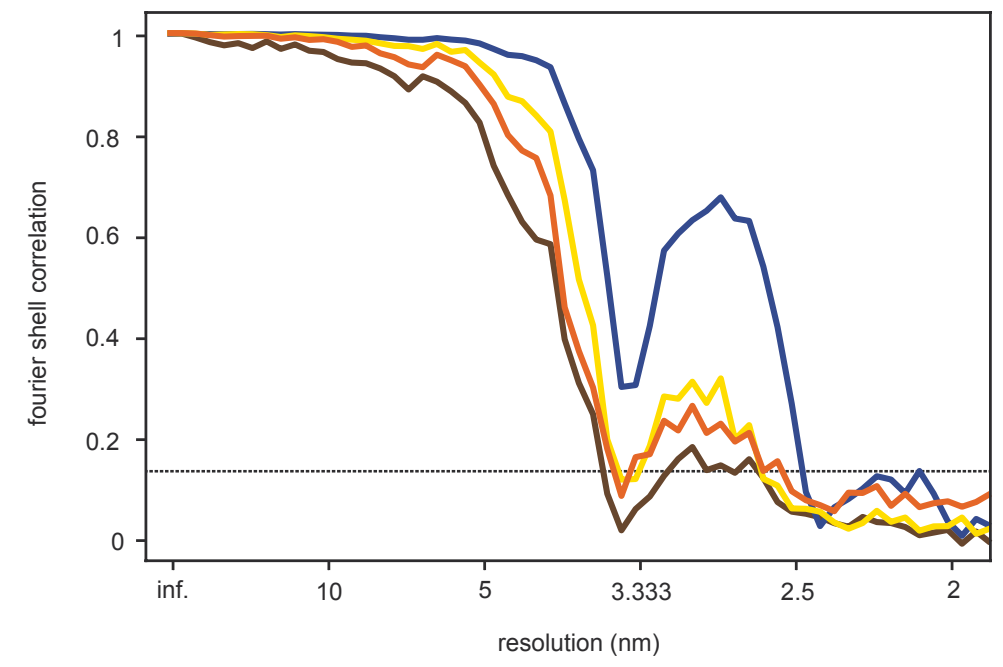

pre-pre $(n=827)$

pre-post $(n=342)$

post-pre $(n=98)$

post-post $(n=592)$

\section{Figure S10: Resolution estimation of ODAs and 96-nm repeat subtomogram averaging maps}

(A) Fourier Shell Correlation (FSC) curves from 96-nm repeat and ODA averages provides an estimation of the reliability of the alignment performed on the different datasets and an estimation of their resolution at the typical 0.143 cut-off (indicated by the horizontal dashed line). The inverse of the spatial frequency at which the FSC first crosses the cut-off defines the resolution. The higher the spatial frequency, the better the structural resolution. The inverse of the spatial frequency is here expressed in nm. (B) FSC curves from class averages generated from filtered wildtype and Ttll3${ }^{-}{ }^{-} t l l 8^{-/}$particles. The reduction in particle number due to the splitting of the dataset into classes still does not hinder dynein heavy-chain resolvability. 
Table S2: Antibodies used for immunoblotting and immunocytochemistry

\begin{tabular}{|c|c|c|c|c|}
\hline $\begin{array}{l}\text { Primary } \\
\text { Antibody }\end{array}$ & Reference $n^{\circ}$ & Antigen & $\begin{array}{l}\text { Dilution } \\
\text { (Western blot) }\end{array}$ & $\begin{array}{l}\text { Dilution } \\
\text { (Immuno- } \\
\text { cytochemistry) }\end{array}$ \\
\hline Gly-pep1 & AdipoGen AG-25B-0034 & $\begin{array}{l}\text { Branch point glycylation } \\
\text { on } \beta \text {-tubulin C-terminal } \\
\text { tail, rabbit polyclonal }\end{array}$ & $1: 10,000$ & $1: 15,000$ \\
\hline $6-11 B-1$ & Sigma Aldrich T7451 & $\begin{array}{l}\text { acetylated } \alpha \text {-tubulin from } \\
\text { the outer arm of sea } \\
\text { urchin, mouse } \\
\text { monoclonal. }\end{array}$ & ---------- & $1: 1000$ \\
\hline PolyE & Adipogen AG-25B-0030 & $\begin{array}{l}\text { linear chains of } 4 \text { and } \\
\text { more glutamate residues, } \\
\text { rabbit polyclonal }\end{array}$ & $1: 10,000$ & $1: 10,000$ \\
\hline $12 \mathrm{G} 10$ & $\begin{array}{l}\text { Developed by J. Frankel and } \\
\text { M. Nelson, obtained from the } \\
\text { Developmental Studies } \\
\text { Hybridoma Bank, developed } \\
\text { under the auspices of the } \\
\text { NICHD, and maintained by the } \\
\text { University of Iowa. }\end{array}$ & $\begin{array}{l}\alpha \text {-tubulin, mouse } \\
\text { monoclonal }\end{array}$ & $1: 500$ & ---------- \\
\hline Arl13b & NeuroMab 75-287 & $\begin{array}{l}\text { Fusion protein amino } \\
\text { acids } 208-427 \text { (C- } \\
\text { terminus) of mouse } \\
\text { Arl13b, mouse } \\
\text { monoclonal }\end{array}$ & --------- & $1: 1,000$ \\
\hline DNAH8 & Sigma Aldrich HPA028447 & $\begin{array}{l}\text { Recombinant axonemal } \\
\text { dynein heavy chain } 8 \\
\text { epitope with signature } \\
\text { tag (PrEST), rabbit } \\
\text { polyclonal }\end{array}$ & -------- & $1: 500$ \\
\hline DNAH17 & Sigma Aldrich HPA024354 & $\begin{array}{l}\text { Recombinant axonemal } \\
\text { dynein heavy chain } 17 \\
\text { epitope with signature } \\
\text { tag (PrEST), rabbit } \\
\text { polyclonal }\end{array}$ & -------- & $1: 200$ \\
\hline DNAH2 & Sigma Aldrich HPA067103 & $\begin{array}{l}\text { Axonemal dynein heavy } \\
\text { chain } 2 \text {, rabbit polyclonal }\end{array}$ & -------- & $1: 100$ \\
\hline DNALI1 & Sigma Aldrich HPA028305 & $\begin{array}{l}\text { Recombinant axonemal } \\
\text { dynein light intermediate } \\
\text { chain } 1 \text { epitope with } \\
\text { signature tag (PrEST), } \\
\text { rabbit polyclonal }\end{array}$ & -------- & $1: 50$ \\
\hline DM1A & Sigma Aldrich T9026 & $\begin{array}{l}\alpha \text {-tubulin, mouse } \\
\text { monoclonal }\end{array}$ & -------- & $1: 500$ \\
\hline
\end{tabular}




\begin{tabular}{|c|c|c|c|c|}
\hline $\begin{array}{l}\text { Secondary } \\
\text { Antibody }\end{array}$ & Reference ${ }^{\circ}$ & Antigen & $\begin{array}{l}\text { Dilution } \\
\text { (Western blot) }\end{array}$ & $\begin{array}{l}\text { Dilution } \\
\text { (Immuno- } \\
\text { cytochemistry) }\end{array}$ \\
\hline $\begin{array}{l}\text { Goat-anti rabbit } \\
\text { IgG-HRP }\end{array}$ & $\begin{array}{l}\text { Bethyl Laboratories Inc. A120- } \\
201 P\end{array}$ & Rabbit whole IgG & $1: 10,000$ & --------- \\
\hline $\begin{array}{l}\text { Goat-anti } \\
\text { mouse IgG- } \\
\text { HRP }\end{array}$ & $\begin{array}{l}\text { Bethyl Laboratories Inc. A90- } \\
516 \mathrm{P}\end{array}$ & Mouse whole IgG & $1: 10,000$ & --------- \\
\hline $\begin{array}{l}\text { Goat-anti } \\
\text { mouse IgG } \\
\text { Alexa Fluor } \\
488\end{array}$ & $\begin{array}{l}\text { Thermo Fisher Scientific. } \\
\text { A11001 }\end{array}$ & $\begin{array}{l}\text { Mouse Gamma } \\
\text { immunoglobins heavy } \\
\text { and light chains }\end{array}$ & -------- & $1: 1,000$ \\
\hline $\begin{array}{l}\text { Goat-anti } \\
\text { mouse IgG2a } \\
\text { Alexa Fluor } \\
488\end{array}$ & $\begin{array}{l}\text { Thermo Fisher Scientific. } \\
\text { A21131 }\end{array}$ & Mouse IgG 2a & -------- & $1: 1,000$ \\
\hline $\begin{array}{l}\text { Goat-anti rabbit } \\
\text { IgG Alexa } \\
\text { Fluor } 568\end{array}$ & $\begin{array}{l}\text { Thermo Fisher Scientific. } \\
\text { A11036 }\end{array}$ & $\begin{array}{l}\text { Rabbit Gamma } \\
\text { immunoglobins heavy } \\
\text { and light chains }\end{array}$ & --------- & $1: 1,000$ \\
\hline
\end{tabular}




\section{Legends to Movies}

\section{Movie S1: Flagellar beat of sperm tethered at their heads}

Dark-field microscopy of head-tethered sperm recorded at 250 frames-per-second (fps). (A) Symmetric and asymmetric flagellar beat for wildtype and $T t l l 3^{-/} T t l l 8^{-/}$sperm, respectively (top). The symmetry of the beat is clearly shown in the corresponding colour-coded flagellar beat projections (bottom panels from Fig 3A). (B-D) Flagellar beat analyses of tethered sperm (panels from Fig 3B-D): ttll3 $^{--}$Ttll ${ }^{-/-}$sperm have $\sim 3.3$ times higher curvature (B) and $\sim 2.4$ times lower beat amplitude (C). The beat frequency (D) is asymmetric: $\sim 3.1$ times lower beat frequency near the head and $\sim 2.7$ times higher frequency in the rest of the flagellum.

\section{Movie S2: Swimming behaviours of sperm}

Dark-field microscopy of free-swimming sperm from wide-field images $(\mathbf{A}, \mathbf{B})$ were recorded at 50 fps. Single-sperm transitions (C-E) were recorded at $250 \mathrm{fps}$. Wildtype sperm swim almost exclusively along an elongated curvilinear path (A,C). In contrast, most $\mathrm{Ttll3}^{-/-} \mathrm{Ttll}^{-/-}$sperm $(\sim 86 \%)$ swim in circular paths $(\mathbf{B}, \mathbf{E})$, with a small fraction $(\sim 14 \%)$ showing incidental progressive motility (B,D).

\section{Movie S3: Helical and circular swimming of $\mathrm{Ttll}^{-/-} \mathrm{Ttll}^{-/-}$sperm}

Dark-field microscopy of free-swimming sperm recorded at $250 \mathrm{fps}$ in custom-made observation chambers of $100 \mu \mathrm{m}$ depth. Upon reaching the wall of the observation chamber, the $\mathrm{Ttll}^{-/-} \mathrm{Ttll} \mathrm{8}^{-/-}$ sperm transitions from helical to circular swimming (still panel from Fig 4D).

\section{Movie S4: Absence of glycylation results in altered conformations of axonemal ODA heavy chains}

(A) Fitting the crystal structures of the dynein motor domain onto isosurface renderings of the 96-nm repeats in sperm axoneme shows an altered conformation of both $\beta$-heavy chain (magenta) and $\gamma$ heavy chain (green) when the dynein shifts from pre-powerstroke to the post-powerstroke condition. (B) Predominant conformations of the ODAs observed in the wildtype and $T t l l 3^{-/}$Ttll $^{-/-}$sperm (panels from Fig 6A). (C) Comparison of the transition of the ODAs shows a coordinated transition from pre-powerstroke (blue) to post-powerstroke (yellow) in the wildtype axonemes, whereas in the Ttll3 ${ }^{-/}$Ttll ${ }^{-/}$axonemes intermediate conformations, pre-post (orange) and post-pre (brown) are more commonly found (bottom panels from Fig 6B). 


\section{Movie S5: IDA-f conformational reconfiguration is linked to the tilting motion of the nexin- dynein regulatory complex (N-DRC)}

(A) Slices through class averages generated from IDA classification showing their overall transition between pre-powerstroke and post-powerstroke conformations (left). Particularly consistent rearrangement of IDA-f subspecies (encircled in red) between the two classes are shown in the right panels (from Fig S8B). (B) The conformational reconfiguration of IDA-f occurred synchronously with an opposing tilting motion of the nexin-dynein regulatory complex and the radial-spoke heads. This tilting motion was perpendicular to the MT axis and associated with a displacement of the next MT doublet. (A tub: A-tubule; B tub: B-tubule; N-DRC: nexin-dynein regulatory complex; RS: radial spoke; ODA: outer dynein arm; IDA: inner dynein arm)

\section{Movie S6: Absence of glycylation has no apparent effect on the beating of ependymal cilia}

(A) Representative video recordings of ependymal cilia acquired from 100-200- $\mu \mathrm{m}$ sagittal sections of wildtype and $\mathrm{Ttll}^{-/} \mathrm{Ttll}^{-/-}$brain ventricles. Recordings were performed at $100 \mathrm{fps}$ for $3 \mathrm{~s}$ using a $60 \times$ objective. The videos were slowed down $20 \times$ to scrutinize the ciliary beat waveform (one representative video in original speed is shown on the left). Visual inspection does not reveal any changes in the beat patterns of multicilia in the absence of glycylation. (B) Scatter plots showing the analysis of cilia beat frequencies from three independent experiments with each point representing an individual cell recorded from the same brain slice (number of cells are given). Median $( \pm \mathrm{SEM})$ is indicated, p-values are determined by Student's t-test. All three experiments coherently show no differences between wildtype and $T t l l 3^{-/} T t l l 8^{-/-}$cells. Note that overall values vary between different experimental days, most likely due to slight variations in imaging conditions (e.g. temperature). 